\title{
Dissipation-Induced Instability Phenomena in Infinite-Dimensional Systems
}

\author{
Rouslan Krechetnikov \& Jerrold E. Marsden
}

\begin{abstract}
This paper develops a rigorous notion of dissipation-induced instability in infinite dimensions as an extension of the classical concept implicitly introduced by Thomson and Tait for finite degree of freedom mechanical systems over a century ago. Here we restrict ourselves to a particular form of infinite-dimensional systems-partial differential equations-whose inherent function-analytic differences from finite-dimensional systems make uncovering this notion more intricate. In building the concept of dissipation-induced instability in infinite dimensions we found Arnold's and Yudovich's nonlinear stability methods, for conservative and dissipative systems respectively, along with some new existence theory for solutions, to be the essential foundation. However, when proving the results for classical solutions, as motivated by their direct physical significance, we had to overcome a number of fundamental difficulties associated with existing stability analysis methods, which has led to new techniques. In particular, in this work we establish the connection of existence and general stability theories in strong and weak topologies and provide new insights into the physics and geometry of the dissipation-induced instability phenomena in infinite-dimensional systems. As a paradigm and the first infinite-dimensional example to be rigorously analyzed, we use a two-layer quasi-geostrophic beta-plane model, which describes the fundamental baroclinic instability in atmospheric and ocean dynamics; early formal linear approximate studies suggested that this system can be destabilized after the introduction of dissipation.
\end{abstract}

\section{Contents}

1. Introduction . . . . . . . . . . . . . . . . . . . 612

1.1. The physical and geometrical picture in finite dimensions . . . . . . . . . 612

1.2. Passage to infinite dimensions and paper outline . . . . . . . . . . . . . . 616

2. Paradigm and the main theorem . . . . . . . . . . . . . . 618 
2.1. Problem statement . . . . . . . . . . . . . . . . . . . . . . . . 618

2.2. Physics of the model . . . . . . . . . . . . . . . . . . . . . . . 619

2.3. The function space setting . . . . . . . . . . . . . . . . . . 620

2.4. A main result . . . . . . . . . . . . . . . . . . . . . . . . . 622

3. Hamiltonian structure . . . . . . . . . . . . . . . . . . . . 625

4. Linear spectral stability analysis . . . . . . . . . . . . . . . . . . . 628

5. Nonlinear stability analysis . . . . . . . . . . . . . . . . . . . 630

5.1. The Hamiltonian case: Arnold's method . . . . . . . . . . . . . . . 631

5.2. The dissipative case . . . . . . . . . . . . . . . . . . . . . . 634

5.3. Discussion: geometrical and physical interpretations . . . . . . . . . . . 643

6. Existence of classical solutions . . . . . . . . . . . . . . . . . . . . 644

6.1. Local existence in the Hamiltonian case . . . . . . . . . . . . . . . . . 647

6.2. Local existence in the dissipative case . . . . . . . . . . . . . . . . . 651

6.3. Global existence in the Hamiltonian case . . . . . . . . . . . . . . . . . 652

6.4. Global existence in the dissipative case . . . . . . . . . . . . . . . . . 655

7. Proof of Theorem 1 and discussion . . . . . . . . . . . . . . . 655

Appendix A: The origin of the quasi-geostrophic model . . . . . . . . . . . . . . . 657

Appendix B: Particle path estimates (6.20) . . . . . . . . . . . . . . . . . . . . . 660

Appendix C: Vorticity estimates $(6.23) \ldots \ldots \ldots 662$

Appendix D: Vorticity estimates with dissipation (6.34) . . . . . . . . . . . . 663

\section{Introduction}

The phenomenon of dissipation-induced instabilities in finite-degree of freedom mechanical systems was known at the end of the nineteenth century to THOMson and TAIT [83], who made it precise by formulating theorems which were later proved by Chetayev [16]. The corollary of these theorems relevant to our discussion states that if a system with an unstable potential energy has been (spectrally) stabilized with gyroscopic forces, then this stability is lost after the addition of arbitrarily small dissipation. As has been recently realized [48], there is another major type of such phenomena, which together with the type studied by THOMson and TAIT exhaust all the generic possibilities, hinted at by Merkin's work [61]: namely, $a$ stable purely potential system with equal frequencies becomes unstable after the addition of arbitrarily small positional forces. The importance of these phenomena in many physical and engineering applications should not be underestimated: the destabilizing effect of dissipation needs to be compensated. For example, in various gyroscopic devices this is done by applying accelerating forces. At the level of every-day experience, the familiar instability of a penny rolling along a straight line is one of numerous illustrations of this phenomenon: if the speed of rolling is high enough then the motion is stabilized gyroscopically, but rolling friction destabilizes it, as we know from experience and modeling.

\subsection{The physical and geometrical picture in finite dimensions}

First, we briefly review the physical significance of the classical finitedimensional theories of Thomson and Tait [83], Chetayev [16], and Merkin [61]. The synthesis of these two theories suggests a general definition of dissipationinduced instability that we give below. For an extended discussion we refer the reader to [48]. 


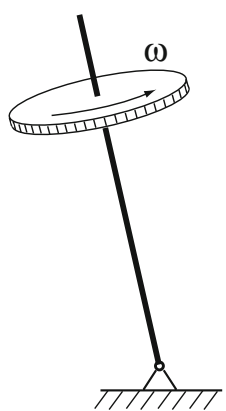

(a)

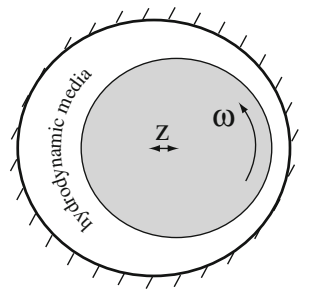

(b)

Fig. 1. Representative examples in finite dimensions: a Lagrange top; b Rotating shaft

To illustrate the above corollary of the Thomson-Tait-Chetayev theory and to appeal to the reader's intuition, we consider the following simple two degree of freedom example ${ }^{1}$ as occurs in, for instance, the linearized dynamics of a Lagrange top which is shown in Fig. 1a,

$$
\begin{aligned}
& \ddot{z}_{1}+g \dot{z}_{2}+d \dot{z}_{1}+c_{1} z_{1}=0, \\
& \ddot{z}_{2}-g \dot{z}_{1}+d \dot{z}_{2}+c_{2} z_{2}=0,
\end{aligned}
$$

where $\mathbf{z}=\left(z_{1}, z_{2}\right)$ is a perturbation which has an unstable equilibrium at the origin if $g=0$ and $c_{i}<0$, but can be stabilized by the gyroscopic forces if $|g|>\sqrt{-c_{1}}+\sqrt{-c_{2}}$ as illustrated by trajectories on the graph of the potential function $V=\frac{1}{2} \mathbf{z} C \mathbf{z}, C=\operatorname{diag}\left[c_{1}, c_{2}\right]$ in Fig. 2a. As one can easily see, the addition of arbitrarily small dissipative forces, $d>0$, that is, a symmetric term proportional to the velocity $\dot{\mathbf{z}}$, destabilizes the equilibrium and leads to the behavior shown in Fig. 2b. As pointed out in [9], the fact that the second variation of the Hamiltonian $H=\frac{1}{2} \dot{\mathbf{z}}^{T} \dot{\mathbf{z}}+\frac{1}{2} \mathbf{z C} \mathbf{z}$ is indefinite is crucial for the destabilizing effect of dissipation, since the condition necessary for stability of a Hamiltonian system, namely definite second variation $\delta^{2} H$ in the Lagrange-Dirichlet theorem, is not satisfied.

The system (1.1) accounts for the dynamics of the perturbation $\mathbf{z}$, so that in this approximation the stability of the equilibrium $\mathbf{z}=0$ can be ascertained. Note that the physical system has a relative equilibrium (that is, the top is in steady rotation about its vertical axis) due to which a gyroscopic force appears in (1.1). Therefore, the model (1.1) accounts for an instability of the top which develops due to dissipative forces. It is clear that dissipation in this case leads to the decay of total energy (that is, the top slows down and eventually falls) as well as to the decrease of the energy of the perturbation (that is, $\dot{H}<0$ ). The latter is allowed by, in this case, an unstable potential energy, cf. Fig. 2 b.

The above situation, accounted for by the Thomson-Tait-Chetayev theory, does not exhaust all the possibilities for the dissipation-induced instability phenomena [47]. Many studies in the earlier part of the twentieth century demonstrated that

\footnotetext{
${ }^{1}$ Unless otherwise stated all variables and coefficients are real.
} 


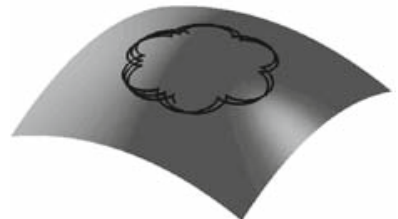

(a)

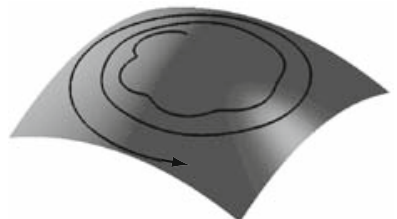

(b)

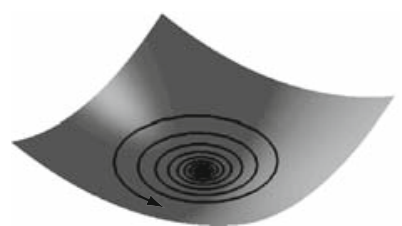

(c)

Fig. 2. Projection of dynamics onto potential energy surface: a Gyroscopically stabilized system with an unstable potential; b Destabilization of gyroscopically stable system; c Destabilizing effect of positional forces

dissipative forces, that is, those that are proportional to velocities with the coefficient matrix being symmetric, are not the only physically important ones of a nonconservative type (in the usual sense that the work done can be path dependent). Another physically significant and widespread class of nonconservative forces includes so-called positional forces, which are proportional to displacements with skew-symmetric coefficient matrix. Their theoretical basis was established by MerKIN $[60,61]$, who proved a number of fundamental properties for the effects of these forces including the corollary stated at the beginning of this introduction. The equal frequencies can come about, for instance, because of a system symmetry. To illustrate Merkin's theory, consider the following system,

$$
\begin{aligned}
& \ddot{z}_{1}+p z_{2}+c z_{1}=0, \\
& \ddot{z}_{2}-p z_{1}+c z_{2}=0,
\end{aligned}
$$

which describes, amongst other systems, the linearized dynamics of a perturbation $\mathbf{z}$ of a rotating shaft [43] shown in Fig. 1b. The corresponding characteristic equation shows that the addition of nonzero nonconservative positional forces (that is, $p \neq 0$ ) to a system with a stable potential energy makes it unstable, as shown in Fig. 2c. The origin of the skew-symmetric positional forces lies in the friction between a rotating shaft and hydrodynamic media, which fills the space between shell and shaft, and in the asymmetry of the gap when the shaft is displaced from the axis of symmetry [43]. Analogous to example (1.1), system (1.2) accounts for the evolution of a perturbation $\mathbf{z}$ measuring the departure from equilibrium, which experiences an instability. The equilibrium corresponds to a relative equilibrium of a rotating shaft, due to which the positional forces appear. It is notable that, in contrast to (1.1), the second variation $\delta^{2} H$ of the original system is positive definite at the 
origin and thus the energy of the disturbance eventually grows. ${ }^{2}$ Despite that, the positional forces in this case are dissipative, since the total energy of the physical system - that of basic state plus perturbation-decays under their action, that is, if one stops maintaining the relative equilibria by applying an external source of energy, the shaft will stop rotating. However, it should be kept in mind that not all positional forces are dissipative, as can be seen on the example of an instability of an elastic bar with a follower force [65], even though the linearized dynamics of perturbation is given by the same Equation (1.2). Concluding the discussion of (1.2) we note that the model (1.2) accounts for an instability of the rotating shaft regardless of whether the relative equilibrium is maintained or not.

Thus, in the finite-dimensional case, the fundamental difference in the type of nonconservative forces, that is, dissipative versus positional (which are also dissipative in nature), has a direct impact and goes in parallel with a drastic change in the geometrical picture, that is, indefinite versus definite second variation of the Hamiltonian:

$$
\begin{aligned}
& \text { unstable potential } \rightarrow \text { indefinite } \delta^{2} H \rightarrow \text { dissipative forces, } \\
& \text { stable potential } \rightarrow \text { definite } \delta^{2} H \rightarrow \text { positional forces, }
\end{aligned}
$$

where we have summarized the geometric precursor which predetermines the type of nonconservative forces that will be destabilizing. ${ }^{3}$ The common features of the two cases in (1.3) are that the instability develops due to withdrawal of energy from the basic state (that is, the relative equilibrium) and the total energy of the whole physical system would decay if the relative equilibrium is not maintained, while the energy of the perturbation might grow.

Motivated by all these physical considerations, we introduce the following definition of dissipative forces.

Definition 1. A set of nonconservative forces acting on a mechanical system with a relative equilibrium is called dissipative if, under the action of these forces and in the absence of the forces which work against these nonconservative forces in order to maintain the (relative) equilibrium, the total mechanical energy of the whole physical system decreases.

This allows us to define a generalized notion of dissipation-induced instability; namely,

Definition 2. A conservative system with a Lyapunov stable (relative) equilibrium is said to suffer from dissipation-induced instability if the introduction of dissipative forces destabilizes this equilibrium in the Lyapunov sense.

2 This, however, does not prohibit having $\dot{H}<0$ on certain portions of the trajectory as can be readily seen from the energy production rate equation: $H=p\left(z_{1} \dot{z}_{2}-z_{2} \dot{z}_{1}\right)$. These portions of a motion correspond to the trajectories that are temporarily heading towards equilibrium.

3 Some other possibilities might be observed in, for instance, the degenerate case when the second variation vanishes or in the case in which a finite-amplitude instability takes place, which we do not discuss here. 
In each concrete situation, the above definition can be made mathematically precise by defining the function space, in which a solution exists, and the norm, in which Lyapunov (nonlinear) stability and instability hold. This will be done in due course in Section 2.4 when formulating the main theorem, as it becomes particularly important in infinite dimensions.

\subsection{Passage to infinite dimensions and paper outline}

The well-established picture and analysis in finite dimensions-where (i) one can relate the origin of a particular type of dissipation-induced instability to the physical type of destabilization forces, (ii) one can enjoy the robust stability analysis methods such as Lyapunov's indirect method, and (iii) one does not need to worry about the dependence of the stability notion on the norm in which solutions existare not readily translated to infinite dimensions. These three main complications are discussed below (for further discussion we refer to KRECHETNIKOV and MARSDEN [48]) and overcoming them is the essential constituent of the main result of this work formulated in Section 2.4. Here we restrict our consideration to systems that cannot be readily approximated with or reduced to some finite-dimensional mechanical analog, and as a paradigm we study the quasi-geostrophic two-layer $\beta$-plane model stated in Section 2.1, which may be viewed as a toy model for the Euler equations in the zero dissipation case, and for the Navier-Stokes equations (NSEs) in the nonzero dissipation case; its physics is discussed in Section 2.2. This class of problems we call essentially infinite-dimensional. Simpler cases have been studied in the literature; for example, a physically interesting system describing radiation-induced instability modeled by HAGERTY ET AL. [36] can be reduced to a low-dimensional system of linear ODEs and thus accounted for by the theory of Section 1.1, as was shown in [47].

The first complication is due to the fact that the corresponding field equations, such as the Euler or NSEs, are usually written not in Lagrangian but rather in Eulerian variables, which results from symmetry reduction, that is, the removal of the particle relabeling symmetry of the original infinite-dimensional system in Lagrangian variables. Therefore, one cannot readily identify the type of forces in the infinite-dimensional case and thus, as the first and natural step to overcome this difficulty, we utilize the fact that the second variation of the Hamiltonian $\delta^{2} H$ goes in parallel with classification of causes for dissipation-induced instabilities (1.3). The Hamiltonian structure of the problem is studied in Section 3.

The second complication results from the lack of a generally applicable analog of the infinite dimensional version of Lyapunov's indirect method (based on the spectrum of the linear approximation), which at least in the finite-dimensional case allows one to conclude nonlinear (in)stability in the dissipative case based on the linear stability picture. We overcome this difficulty using the semigroup theory and a priori estimates in Section 5.2, where we establish nonlinear (in)stability based on the results of the linear theory given in Section 4. Also, in Section 5.2 we establish the result which will allow us to prove instability in a strong topology, which is unconditional upon existence of a solution, based on conditional stability in a weak topology and local existence in the same strong topology. In the Hamiltonian case 
though, the situation with proving stability is similar to that in finite dimensionsone has to prove nonlinear stability without relying on linear stability results, since linear stability need not imply nonlinear (Lyapunov) stability. The latter is because of the well-known fact that the only way for an equilibrium of a Hamiltonian system to be stable is to have all eigenvalues on the imaginary axis since the eigenvalues in the Hamiltonian case come in pairs and quadruples. We will analyze Hamiltonian stability in Section 5.1 with the help of the ARNOLD method [5,6].

The third complication comes from the necessity to establish the link between existence and nonlinear stability, which is impeded by the fact that these two analyses are often done in different functional settings. ${ }^{4}$ Hence, many works written on nonlinear stability of PDEs (for example, [53,54,62]) are conditional upon existence of solutions, with a few exceptions, for special situations (such as WAN and Pulvirenti [85], Burton [11]). Here we restrict ourselves to the existence of classical $^{5}$ solutions where, as we shall see, stability methods and existence theory overlap. The existence of solutions is studied separately in Section 6. Since the purpose of our study is not to advance the existence theory for quasi-geostrophic equations in any significant way, but rather to demonstrate rigorously (unconditionally) the presence of a dissipation-induced instability phenomenon, we prove local existence for rather mild initial conditions in $\mathcal{C}^{1}$ for Hamiltonian and dissipative cases in Sections 6.1 and 6.2 respectively. The global existence in the Hamiltonian case is demonstrated in Section 6.3 under the additional assumption of boundedness of the initial data in the Sobolev space ${ }^{6} \mathcal{H}^{s}, s \geqq 3$. Global existence in the dissipative case is trivial below the critical point and unnecessary above it, which is discussed in Section 6.4. Connecting the existence to the stability results in Section 7 will complete the proof of the main theorem stated precisely in Section 2.4. All existence theorems are proved using a priori estimates and standard techniques in PDEs. It should be mentioned that the known proofs of existence for quasi-geostrophic equations are given for doubly periodic spatial domains [7] and for weak solutions $[10,19,24]$, while here we treat classical solution(s) on a compact domain with one dimension being periodic and one with solid boundaries (channel).

4 That natural connection might be possible to establish using a variational proof of existence, that is, considering the resulting PDEs as Euler-Poincaré equations with the appropriate variational structure, since nonlinear stability method based on the use of Hamiltonian formulation is dual to the variational formulation. However, a variational proof is not available even for the ideal fluid Euler equation.

5 That is, when all the derivatives which appear in the statement of the PDE exist and are continuous.

6 As mentioned above, the methods used for proving nonlinear stability and solution existence are fundamentally disjoint and, in general, are performed in different functional setups. While we proved the existence of classical solutions, that is, when both stability and existence theories overlap, the lack of elegance and effectiveness becomes obvious. 


\section{Paradigm and the main theorem}

\subsection{Problem statement}

Having set the general scene we now present the formulation of the infinite-dimensional paradigm used in our study: the quasi-geostrophic two-layer $\beta$-plane model introduced by PHILLIPS [71] in 1951, namely

$$
\partial_{t} q_{i}+\mathbf{v}_{i} \cdot \nabla q_{i}=-r \nabla^{2} \psi_{i}, \quad \mathbf{x}=(x, y) \in \mathbf{D}, \quad i=1,2,
$$

with no summation over $i$ (sometimes we drop indeces in the text when no confusion arises between scalar and vector notations). This model, whose notation is explained below, accounts for large-scale evolution in mid-latitudes with the simplified effects of Earth's rotation and sphericity ( $\beta$-effect), stratification (modeled by using twolayers) with internal rotational Froude number $F$, and Eckman layer dissipation $(r \geqq 0)$. The definition of nondimensional parameters is given in Appendix A (see also [68]). Equation (2.1) is posed on a rectangular domain, $\mathbf{D}=\{-1 \leqq x \leqq$ $1 ; 0 \leqq y \leqq 1\}$, located on the surface of a rotating planet as shown in Fig. 3 . Physically, this corresponds to a standard rectangular channel of finite depth in the direction $z$ perpendicular to the Earth's surface, and with finite dimensions both in the west-east (zonal) periodic direction $x$ and in the north-south (meridional) direction $y$ which is bounded by solid walls. In the formulation (2.1), we have used the usual definition of potential vorticity, namely

$$
q_{i}=\nabla^{2} \psi_{i}+(-1)^{i} F\left(\psi_{1}-\psi_{2}\right)+\beta y,
$$

where the stream-functions $\psi_{i}$ in the $i$ th layer is related to the velocities by $\mathbf{v}_{i}=$ $\left(u_{i}, v_{i}\right)=\mathbf{e}_{z} \times \nabla \psi_{i}=\left(-\partial_{y} \psi_{i}, \partial_{x} \psi_{i}\right)$, and where the two-dimensional gradient is $\nabla=\mathbf{i} \partial_{x}+\mathbf{j} \partial_{y}$. For convenience, we will also be using vector notation both for potential vorticity, $\mathbf{q}=\left(q_{1}, q_{2}\right)$, and stream-functions, $\psi=\left(\psi_{1}, \psi_{2}\right)$. The basic state of (2.1), in our case the time-independent solution $\psi_{i}^{e}=-U_{i} y$ shown in Fig. 3, will be denoted by $\boldsymbol{\psi}^{e}$ or, equivalently, by $\mathbf{q}^{e}$, since the latter is expressed in terms of $\boldsymbol{\psi}^{e}$. Similarly, the initial conditions are denoted by $\mathbf{q}_{0}$. The left-hand side of (2.1) is the usual material (Euler) transport of potential vorticity (for example, HoLm [40]), while the right-hand side is the Eckman layer dissipation, where $r \geqq 0$.

The problem (2.1) is treated here with the boundary conditions corresponding to the Phillips model. That is, since the channel is periodic in $x$, the appropriate boundary conditions correspond to $D^{\alpha} \psi_{i}(x, y),|\alpha| \geqq 0$ being periodic in $x$ with period 2. Also, since the channel is bounded by walls at $y=0,1$, a no-penetration condition applies,

$$
v=\psi_{x}=0 \text { for } y=0,1,
$$

which implies that the stream-function is only a function of time at the solid walls. As first established by PHILLIPS [72], there is another boundary condition at $y=$ 0,1 , namely

$$
\frac{\partial}{\partial t} \Gamma_{i}(y)=0, \quad \Gamma_{i}(y)=\int_{-1}^{1} u_{i} \mathrm{~d} x
$$




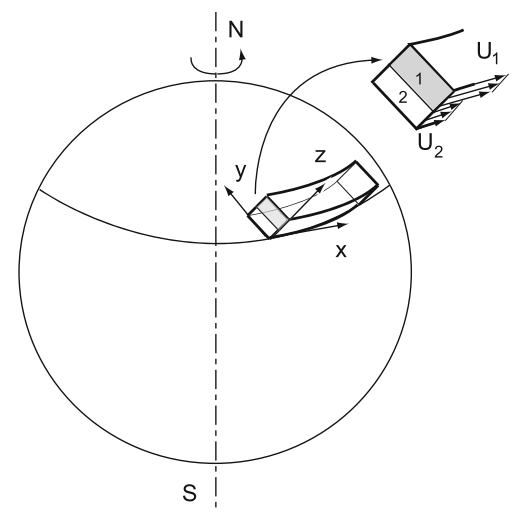

Fig. 3. Physical domain and basic state $U_{i}$ on the surface of a rotating planet

which formally guarantees uniqueness (otherwise the no-penetration condition is satisfied by an arbitrary function of time $t$ ), and loosely can be interpreted as the conservation of "circulations" $\Gamma_{i}(y)$ at the solid boundaries. The main problem is then to ascertain the (in)stability of the basic state in a suitable norm. This completes the problem statement: from now on we are working with system (2.1) and the boundary conditions (2.2-2.3). Though we discuss only this case, the main result of this paper remains valid for a wider class of boundary conditions, such as doubly periodic perturbations imposed on the same basic state $\psi_{i}^{e}$ as considered here. A brief self-contained derivation of (2.1) and boundary condition (2.3) is provided in Appendix A.

\subsection{Physics of the model}

The model (2.1) has been studied for a long time in view of its physical importance in ocean and atmospheric dynamics. ${ }^{7}$ In particular, (2.1) was suspected to have a dissipation-induced instability [18] based on a classical linear stability study [75], but the proof was absent. The main feature of (2.1) relevant to our study is its ability to account for the so-called baroclinic instability - a large-scale instability of the westerly winds in mid-latitudes when the basic (equilibrium) state has a latitudinal shear, $\psi_{i}^{e}=-U_{i} y$, as shown in Fig. 3. In this mathematical idealization, the origin of the basic state is unspecified and the model reflects the fact that this basic state is maintained against dissipative effects by an external source of energy. Physically, this particular basic state results from a temperature gradient between the subtropical and polar regions, which causes a pressure gradient aloft. The latter is balanced by the Coriolis force to form a geostrophic flow, namely the Westerlies. The instability of these baroclinic zonal currents has been a subject of numerous studies starting with pioneering works by CHARNEY and ELIASSEN [14] and EADY

${ }^{7}$ It is notable that the famous Lorentz system is derivable from this model: see GiBBoN and McGuinness [33] and WiIn-Nielsen [87]. 
[25], and is known to occur as a result of a release of available potential energy of sloping density surfaces. The significance of the baroclinic instability comes from the fact that it is responsible for the mid-latitude cyclogenesis, in particular. The general physics of this instability was reviewed by HART [37] and PIERREHUMBERT and SWANSON [74]. While the simplest Eady model explains the basic mechanism, the Charney model is more realistic since it includes the $\beta$-effect, the importance of which has been understood since the work of PHILLIPS [71].

The unexpected destabilizing effect due to the introduction of dissipation was suggested by the linear stability studies of HoLOPAINEN [42] and RomeA [75]. The latter work was based on the PHILLIPS model [72], that is, conducted on an infinite channel domain for which one of the spatial variables is bounded and the other is unbounded, and in a two-layer approximation. Romea demonstrated that an introduction of dissipation leads to an $O(1)$ destabilization effect. While the linear stability analysis is very attractive for an infinite channel, the unbounded geometry introduces a number of complications: (a) unbounded total energy of the system, (b) the function space corresponding to disturbances, which decay at infinity, is different from the one considered by Romea, who restricted the analysis to the bounded functions. However, the fact that the baroclinic instability is a finite wave-number instability allows one to consider the problem on a compact domain without substantial modification of the physics. Besides the above mentioned works concerning a linear stability analysis, the baroclinic instability has also been studied in a finite-amplitude stability realm by PEDLOSKy [68,69] and RoMEA [75] to mention a few, and a nonlinear Hamiltonian stability analysis of the quasi-geostrophic equations has been conducted by Pierini and VulPiani [73], Holm et al. [41], and SWATERS [82]. However, no attempt to prove the presence of a dissipation-induced instability in a sense of Definition 2 has been made.

\subsection{The function space setting}

Throughout the discussion in this study, we will be working in, besides a Banach space $X$ for the general theory, the following specific function spaces and norms which will be used for making the stability and existence of solutions precise.

First, a classical solution will be naturally understood with the help of Hölder spaces ${ }^{8} \mathcal{C}^{s, \lambda}$, that is, spaces of functions $f: \mathbf{D} \rightarrow \mathbb{R}$ bounded and continuous together with their spatial derivatives of order $|\alpha| \leqq s \in \mathbf{Z}_{+}$, where the derivatives of order $s$ are uniformly Hölder continuous with exponent $\lambda \in(0,1]$. The corresponding norm in $\mathcal{C}^{s, \lambda}(\mathbf{D})$ is chosen to be the supremum $\left(L^{\infty}\right)$ norm on derivatives and is given by

$$
\|f(\mathbf{x})\|_{\mathcal{C}^{s, \lambda}}=\sum_{|\alpha| \leqq s} \sup _{\mathbf{x} \in \mathbf{D}}\left|D^{\alpha} f(\mathbf{x})\right|+\sum\left[D^{s} f(\mathbf{x})\right]_{\lambda},
$$

8 We avoid using the notation $\mathcal{C}^{s+\lambda}$ adopted by some authors since, for example, $\mathcal{C}^{1+0}$ is not the same as $\mathcal{C}^{0+1}$. 
where we used the definition of a function $\phi(\mathbf{x}) \in C^{0}(\mathbf{D})$, which is Hölder continuous on $\mathbf{D}$ with coefficient $\lambda \in(0,1)$ and with finite semi-norm:

$$
[\phi(\mathbf{x})]_{\lambda}=\sup _{\substack{\mathbf{x}^{\prime}, \mathbf{x}^{\prime \prime} \in \mathbf{D} \\ \mathbf{x}^{\prime} \neq \mathbf{x}^{\prime \prime}}}\left\{\frac{\left|\phi\left(\mathbf{x}^{\prime}\right)-\phi\left(\mathbf{x}^{\prime \prime}\right)\right|}{\left|\mathbf{x}^{\prime}-\mathbf{x}^{\prime \prime}\right|^{\lambda}}\right\} .
$$

Lipshitz continuity corresponds to the case $\lambda=1$, that is, $\mathcal{C}^{0,1}$ or in general $\mathcal{C}^{s, 1}$. Note, here $D^{\alpha}=\partial_{x}^{\alpha_{x}} \partial_{y}^{\alpha_{y}},|\alpha|=\alpha_{x}+\alpha_{y} \in \mathbf{Z}_{+}$is a multi-index. Also we will be using notation $\mathcal{C}^{s}$ for spaces of functions $f: \mathbf{D} \rightarrow \mathbb{R}$ with bounded and continuous spatial derivatives of order $|\alpha| \leqq s \in \mathbf{Z}_{+}$. It is clear that $\mathcal{C}^{s, \lambda}$ with $\lambda \in(0,1)$ is imbedded in $\mathcal{C}^{s}$ with the appropriate norm

$$
\|f(\mathbf{x})\|_{\mathcal{C}^{s}}=\sum_{|\alpha| \leqq s} \sup _{\mathbf{x} \in \mathbf{D}}\left|D^{\alpha} f(\mathbf{x})\right| \leqq\|f(\mathbf{x})\|_{\mathcal{C}^{s, \lambda}}
$$

Since we are working with time-dependent functions, the space $\mathcal{C}^{s, \lambda}$ is generalized to $\widehat{\mathcal{C}}_{0}^{s, \lambda}(\mathbf{D} \times[0, T])$ for functions $f(\mathbf{x}, t)$ belonging to $\mathcal{C}^{s, \lambda}$ uniformly in $t \in[0, T]$, that is, their spatial derivatives up to order $s$ are continuous with Hölder exponent $\lambda$, with the corresponding norm

$$
\|f(\mathbf{x}, t)\|_{\widehat{\mathcal{C}}_{0}^{s, \lambda}}=\sup _{0 \leqq t \leqq T}\|f(\mathbf{x}, t)\|_{\mathcal{C}^{s, \lambda}}
$$

Note that the space $\widehat{\mathcal{C}}_{0}^{s, \lambda}$ does not require the continuity of temporal derivatives, but only the function itself and its spatial derivatives, which motivates the usage of subindex 0 , while hat ${ }^{-}$indicates that the function is defined on $\mathbf{D} \times[0, T]$. To reflect on the continuity of temporal derivatives too, we also use the space $\widehat{\mathcal{C}}^{s, \lambda}(\mathbf{D} \times[0, T])$ with the norm

$$
\|f(\mathbf{x})\|_{\widehat{\mathcal{C}}^{s, \lambda}}=\sum_{|\alpha| \leqq s} \sup _{(\mathbf{x}, t) \in \mathbf{D} \times[0, T]}\left|D^{\alpha} f(\mathbf{x}, t)\right|+\sum\left[D^{s} f(\mathbf{x}, t)\right]_{\lambda},
$$

where $D^{\alpha}=\partial_{x}^{\alpha_{x}} \partial_{y}^{\alpha_{y}} \partial_{t}^{\alpha_{t}},|\alpha|=\alpha_{x}+\alpha_{y}+\alpha_{t} \in \mathbf{Z}_{+}$. Finally, these definitions are naturally extended to vector-valued functions. The membership of a vector-valued function $\mathbf{f}(\mathbf{x}, t)$ in a function space, $\mathcal{C}^{s, \lambda}, \widehat{\mathcal{C}}_{0}^{s, \lambda}$ or $\widehat{\mathcal{C}}^{s, \lambda}$, implies that every component of the vector belongs to that space.

While the above Hölder spaces are enough for local (in time) existence, the global estimates in Section 6.3 will be made with the use of the Hilbert spaces $\mathcal{H}^{s}(\mathbf{D})$ with generalized spatial derivatives up to order $s$ belonging to the Lebesgue space of square-integrable functions over $\mathbf{D}: L^{2}(\mathbf{D})$ and with the norm defined in a standard way

$$
\|f\|_{\mathcal{H}^{s}}=\sum_{|\alpha| \leqq s}\left\|D^{\alpha} f\right\|_{\mathcal{H}^{0}}
$$

where $\|f\|_{\mathcal{H}^{0}}$ is a norm in $L^{2}(\mathbf{D})$ space.

Finally, for nonlinear stability analysis purposes in Section 5.2, we will also need the Sobolev spaces $W^{s, q}(\mathbf{D})$ of all locally summable functions $f: \mathbf{D} \rightarrow \mathbb{R}$ 
over $\mathbf{D}$ such that for each multi-index $\alpha$ with $|\alpha| \leqq s$, spatial derivatives $D^{\alpha} f$ exist in the weak sense and belong to $L^{q}(\mathbf{D})$. The norm is defined to be

$$
\|f\|_{W^{s, q}(\mathbf{D})}=\left[\sum_{|\alpha| \leqq S} \int_{\mathbf{D}}\left|D^{\alpha} f\right|^{q} \mathrm{~d} x\right]^{1 / q}, \quad 1 \leqq q<\infty .
$$

Occasionally, for convenience, we will abbreviate the above norm notation to $\|f\|_{s, q}$.

\subsection{A main result}

To formulate a main result of our work and to put it in a general context, we first appeal to an alternative point of view on the concept of dissipation-induced instability. As will be shown, the Hamiltonian for system (2.1) is given by

$$
H \equiv \frac{1}{2} \iint_{\mathbf{D}}\left\{F\left(\psi_{1}-\psi_{2}\right)^{2}+\nabla \psi_{i} \nabla \psi_{i}\right\} \mathrm{d} \mathbf{x},
$$

where a summation over $i$ is assumed (we use this Einstein convention in the text unless otherwise stated). One might expect that energy dissipation (that is, $H$ decreases) can be achieved by an increase in the amplitude of the solution $\psi_{2}$ in the second layer. This constitutes the idea of a negative-energy mode known since the example of two coupled oscillators developed by CHERRY [15] and defined in WeILAND and Wilhelmsson [86]; see also the discussion in MorRison [64]. In our case one cannot a priori validate this point of view, because knowledge of the Hamiltonian is not enough to deduce dynamic information and to predict the behavior of the second term in the Hamiltonian (2.9). Moreover, this intuitive reasoning turns out to be incorrect as applied to the quasi-geostrophic system (2.1), and, because the second variation $\delta^{2} H$ is proved to be definite, dissipation-induced instability develops according to scenario (1.3b) in analogy with positional forces in the finite-dimensional case. While we are not suggesting that an infinite dimensional analog of the condition on "equal frequencies" holds here, nevertheless finite dimensional theory suggests that, in the presence of a positive definite second variation, an instability could develop only with the addition of destabilizing effects which are analogous to positional forces in finite dimensions; this will indeed be shown to hold.

To make the formulation of the main theorem mathematically precise we have to take into account the discussion in Section 1.2 and to introduce the necessary functional framework. First, we consider quasi-geostrophic system (2.1) as an infinitedimensional ODE in a Banach space $X$ in analogy with DALECKII and KREIN [22], Yudovich [88], and FriedLANDER et al. [29,30]. Since we are interested in a stability analysis, the solution is decomposed as $\psi_{i}=\psi_{i}^{e}+\phi_{i}$ around the basic state $\psi_{i}^{e}=-U_{i} y$, and the appropriate form of this ODE in an operator formulation is obtained after substitution of the representation of $q_{i}$ in terms of $\psi_{i}$. Doing this, Equation (2.1) takes the Orr-Sommerfeld-like form

$$
\partial_{t} M \phi_{i}+L \phi_{i}=N\left(\phi_{i}\right), \quad i=1,2,
$$


with the following definitions of the operators

$$
\begin{aligned}
\text { mass operator : } & M \phi_{i}=\nabla^{2} \phi_{i}+(-1)^{i} F\left(\phi_{1}-\phi_{2}\right), \\
\text { linear operator : } & L \phi_{i}=U_{i} \partial_{x} M \phi_{i}+\beta \partial_{x} \phi_{i}+r \nabla^{2} \phi_{i}, \\
\text { nonlinear operator : } \quad N\left(\phi_{i}\right) & =\partial_{y} \phi_{i} \partial_{x} M \phi_{i}-\partial_{x} \phi_{i} \partial_{y} M \phi_{i} .
\end{aligned}
$$

The problem (2.10) is posed on a compact domain $\Omega \subset \mathbb{R}^{n}$, where $n=2$ in our case. The difference of (2.10) from the one used by YudovicH [88] is notable because of the presence of the operator $M$, which is an identity in the Yudovich theory $^{9}$ : this has some implications on stability analysis in the dissipative case in Section 5.2.

This approach allows one to use the dynamical theories of DALECKII and KREIN [22], Yudovich [88] for nonlinear stability, when one sees the PDE model (2.10) as an infinite-dimensional ODE in operator form

$$
\frac{\mathrm{d} \phi}{\mathrm{d} t}=\mathcal{A} \boldsymbol{\phi}+\mathcal{N}(\boldsymbol{\phi}, t)
$$

where $\mathcal{A}$ is the linear operator, usually stationary, and $\mathcal{N}$ is the nonlinear operator. The relation between (2.10) and (2.12) will be established in Section 5.2.1. By the solution of system (2.12) we mean a classical solution in the usual sense, that is, all its derivatives, which appear in the PDE statement (2.1), exist and are continuous. The existence of these solutions in $X$ will be proved in Section 6 . The exact specification of the Banach space $X$ will be given later in (6.11); at this point it is clear that $X$ is determined by the boundary conditions discussed in Section 2.1 and the classical regularity of a solution. Given the notion of a solution, we also need suitable versions of Lyapunov (nonlinear) stability and instability, provided by the following definitions, which can be applied both in the context of quasi-geostrophic vorticity in (2.1) and stream-function in (2.10).

The instability of the solutions of (2.12) will be understood in the classical mechanics sense, as it originated historically from the 1892 doctoral dissertation of LYAPUNOV [57]. Since there are variants of this notion in the literature, we recall the definitions, beginning with stability. Let us fix some initial time instant $t_{0}$.

Definition 3. Let $X$ be a Banach space. A solution $\phi^{e}(t) \in X$ to (2.12) is said to be Lyapunov, or nonlinearly, stable if for any $\epsilon>0$ there exists a $\delta>0$ such that for any $T \geqq t_{0}$, and initial conditions $\boldsymbol{\phi}_{0} \in X$ satisfying $\left\|\boldsymbol{\phi}_{0}-\boldsymbol{\phi}^{e}\left(t_{0}\right)\right\|_{X}<\delta$, the following two conditions hold:

(i) there exists a solution $\boldsymbol{\phi}\left(t, t_{0}, \boldsymbol{\phi}_{0}\right)$ for $t \in\left[t_{0}, T\right]$ s.t. $\phi(t) \in C\left(\left[t_{0}, T\right] ; X\right)$;

(ii) $\left\|\boldsymbol{\phi}\left(t, t_{0}, \boldsymbol{\phi}_{0}\right)-\boldsymbol{\phi}^{e}(t)\right\|_{X}<\epsilon$ for all $t \in\left[t_{0}, T\right]$.

${ }^{9}$ In the case of the Euler or the NSEs, one can go back from vorticity formulation to velocity formulation, so that using the Helmholtz decomposition [17] and applying the Leray projector $\mathbb{P}$ onto the space of divergence free (solenoidal) functions, one arrives at the operator form (2.10) with $M$ being identity. However, as one can easily see, Equation (2.1) does not take that standard form. 
This definition is of course not to be confused with HADAMARD stability [35], that is, continuous dependence of the solution on the initial data, when the bound on the initial data $\delta$ would depend on the length $T$ of the time interval.

Since our goal here is to prove a strong form of the negation of this definition, we will need its precise formulation (cf. Demidovich [23], Adrianova [3]). If one takes the literal negation of Definition 3, then a solution $\phi^{e}(t) \in X$ of (2.12) would be called Lyapunov unstable (or simply unstable) even when condition (i) fails, that is, when solutions fail to exist. Then one would be proving what one might call conditional instability; that is, solutions arbitrarily close to equilibrium escape a ball of some positive radius provided that they exist. In this work we will deal with a stronger notion of instability, given below.

Definition 4. A solution $\phi^{e}(t) \in X$ of (2.12) is called strongly Lyapunov unstable if there exists an $\epsilon>0$ such that for any $\delta>0$ there exist a time $t_{1}>t_{0}$ and a solution $\phi\left(\cdot, t_{0}, \phi_{0}\right) \in C\left(\left[t_{0}, t_{1}\right], X\right)$ satisfying the condition

$$
\left\|\boldsymbol{\phi}_{0}-\boldsymbol{\phi}^{e}\left(t_{0}\right)\right\|<\delta
$$

and

$$
\left\|\boldsymbol{\phi}\left(t_{1}, t_{0}, \boldsymbol{\phi}_{0}\right)-\boldsymbol{\phi}^{e}\left(t_{1}\right)\right\|_{X} \geqq \epsilon .
$$

Note that here we used only one Banach space $X$, to which both the initial condition and solution belong and whose norm is used for measuring the instability, while if one wants to demonstrate that the solution becomes smoother with time, two Banach spaces are introduced as is usually done for dissipative problems, cf. Yudovich [88]. With Definitions 3-4, the concept of dissipation-induced instability given by the physical Definition 2 becomes precise in the function-theoretic context.

With this understanding, the main result of our work can be formulated as follows; as mentioned above, the space $X$ used for classical solutions will be made precise later, in Equation (6.11).

Theorem 1. Within the class of classical solutions, the equilibrium

$$
\psi_{i}^{e}=-U_{i} y
$$

of the Hamiltonian quasi-geostrophic two-layer $\beta$-plane system (2.1) without dissipation, $r=0$, experiences a dissipation-induced instability in the parameter range

$$
0.4551 \approx \frac{\sqrt{\sqrt{2}-1}}{\sqrt{2}}<\frac{\left|U_{1}-U_{2}\right| F}{2 \beta}<\frac{1}{2}
$$

in the sense of Definition 2 when an arbitrarily small dissipation effect, $r>0$, is added.

Moreover, in the space of classical solutions of (2.1) in the parameter range (2.14) the equilibrium (2.13) is Lyapunov stable in the sense of Definition 3 for $r=0$, but is strongly Lyapunov unstable in the sense of Definition 4 for any $r>0$.

From the physical viewpoint, this result implies that if one is predicting the appearance of a baroclinic instability by measuring the velocity difference $U_{c}=$ $\left|U_{1}-U_{2}\right|$ based on the Hamiltonian formulation, the error of predicting the 


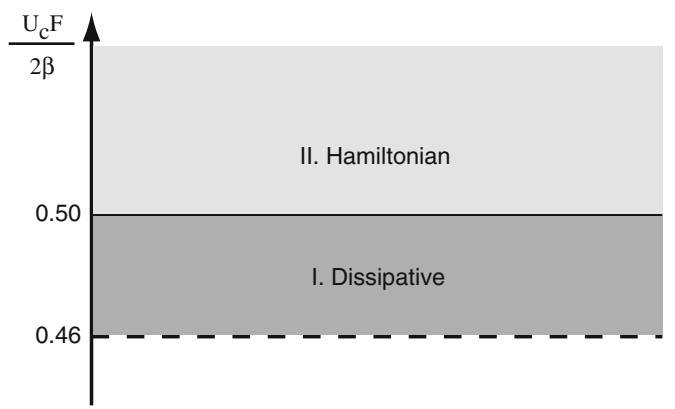

Fig. 4. Sketch of the gap in the criticality properties between the conservative and dissipative systems, which leads to the dissipation-induced instability phenomenon

critical bifurcation parameter will be around $10 \%$. Though this difference is probably within the accuracy of meteorological forecasts, it is still of physical and mathematical importance: we believe that this phenomenon is more frequent than rare and its prominence may vary depending upon a particular problem at hand. Another plausible physical system which may experience this type of instability corresponds to continuously stratified fluid with the effects of shear, buoyancy and rotation [1].

Subsequent sections are devoted to the proof of Theorem 1. To explain the overall strategy behind the proof, we appeal to the sketch in Fig. 4, which depicts the criticality phenomena for the conservative and dissipative cases. Our goal is to demonstrate in the category of classical solutions of (2.1) the presence of a dissipation-induced instability in the sense of Definition 2. To achieve that, we need to prove that a solution in the Hamiltonian case is Lyapunov stable in region I below the solid curve given by $U_{c} F / 2 \beta<\frac{1}{2}$ in Fig. 4 , and that a solution in the dissipative case is Lyapunov unstable in region I above the dashed curve given by $U_{c} F / 2 \beta>\sqrt{\sqrt{2}-1} / \sqrt{2}$ in the same figure. These are the minimal requirements for a dissipation-induced instability to take place, which are addressed in Section 5. In the course of proving this result we will also need to establish enough existence theory in Section 6 so that one can demonstrate the presence of dissipation-induced instability phenomenon not conditioned on the existence of solutions. In fact, as we will see in Section 7, Theorem 1 is a corollary of the corresponding theorems on (in)stability and existence, which are applicable to different function spaces but overlap for the class of classical solutions. Before proceeding to the stability and existence analysis, we will need some knowledge of the linear spectral stability properties, to be studied in Section 4, and the Hamiltonian structure, to be developed in Section 3. The latter will also allow us to investigate the geometrical picture for this particular infinite-dimensional problem as related to the underlying geometry in the finite-dimensional case shown in Fig. 2.

\section{Hamiltonian structure}

In this section we are interested in understanding the energy behavior of the system (2.1) and in determining conservation laws in the form of Casimirs (to 
be defined in due course) which are used in the nonlinear stability analysis in Section 5.1. As a consequence, we first need to develop a Hamiltonian structure for (2.1) starting with the Hamiltonian (2.9). In this section we assume that classical solutions exist for which all the conservations laws make mathematical sense, though they are applicable for a wider class of solutions; their existence will be established in Section 6.

As usual, the equation for the energy is obtained by multiplying each equation in (2.1) with the corresponding stream-function $\psi_{i}$ and summing up. This yields

$$
\partial_{t} H^{\prime}=-r \nabla \psi_{i} \nabla \psi_{i}+\nabla \cdot\left(\psi_{i} \partial_{t} \nabla \psi_{i}+\psi_{i} q_{i} \mathbf{v}_{i}+r \psi_{i} \nabla \psi_{i}\right)
$$

where we have used $H^{\prime}$ as the integrand in the expression for the Hamiltonian (2.9), the elementary identity $\psi \mathbf{v} \cdot \nabla q=\nabla \cdot(\psi q \mathbf{v})-\mathbf{v} \cdot q \nabla \psi=\nabla \cdot(\psi q \mathbf{v})$, and standard differential equalities. Before proceeding with deriving an integral counterpart of (3.1) we make some remarks on the structure of the solution. Since the object of our study is a stability analysis of the basic state $\psi_{i}^{e}$ which represents a vertical shear, we decompose a general solution into the basic state $\psi_{i}^{e}$ and a perturbation $\phi_{i}$

$$
\psi_{i}=\psi_{i}^{e}+\phi_{i}, \quad \text { where } \psi_{i}^{e}=-U_{i} y .
$$

As we will see, in view of the nature of the boundary conditions (2.2-2.3) and the basic state, the perturbation $\phi_{i}$ vanishes at $y=0,1$. Using this fact, the Gauss divergence theorem in the plane and the boundary conditions (2.2-2.3) yield

$$
\frac{\mathrm{d} H}{\mathrm{~d} t}=r \int_{\mathbf{D}}\left\{\psi_{i}^{e} \nabla^{2} \phi_{i}-\left(\nabla \phi_{i}\right)^{2}\right\} \mathrm{d} \mathbf{x},
$$

which is clearly not negative definite. This implies that the effect of Eckman layers is not trivial. Depending on the solution structure and the magnitude of the parameter $r$, as we shall see from the subsequent analysis, an introduction of Eckman layers provides a mechanism for the withdrawal of energy from the basic state (that is, the relative equilibrium) to the disturbance. Similar to the finitedimensional case (1.2), it makes no physical sense to judge whether nonconservative forces are dissipative or not based on the sign of (3.3) since the basic state is maintained by an unlimited external source of energy. The dissipative effect of Eckman layers becomes apparent when the basic state is not maintained by an external source of energy. While one cannot easily see this in our case, that is, when the basic state flow goes through the open channel $\mathbf{D}$, this can be observed when (2.1) is posed on a closed simply connected domain with a solid boundary. In view of the impermeability condition imposed at the solid boundary, $\dot{H}$ becomes negative definite. Thus, any nontrivial initial flow, created by "stirring" for example, will decay to a zero solution provided that $r>0$. Since the effect of Eckman layers does not depend on the boundary conditions at $\partial \mathbf{D}$, one can conclude that their nature is, in general, dissipative; see also the discussion in [70, sections 6.10 and 6.16].

Thus, the case $r=0$ corresponds to the conservative dynamics with the Hamiltonian (2.9). Since there are four more conserved quantities, namely the 
boundary circulations (2.3), the dynamics of (2.1) is restricted to the hypersurface defined by the total energy constrained by the "circulations" $\Gamma_{i}(0,1)(\mathrm{cf} .2 .3)$ :

$$
\widehat{H}=H+\sum_{y=0,1} \lambda_{y}^{i} \Gamma_{i}(y)
$$

where $\lambda_{y}^{i}$ are Lagrange multipliers. The first variation of (3.4) is computed according to $\delta \widehat{H}=\left.\frac{d}{d \epsilon}\right|_{\epsilon=0} \widehat{H}\left(\psi_{i}+\epsilon \delta \psi_{i}\right)=0$, that is

$$
\delta \widehat{H}=-\iint_{\mathbf{D}} \psi_{i} \delta q_{i} \mathrm{~d} \mathbf{x}+\sum_{y=0,1}\left[\left.\int_{-1}^{+1} \psi_{i} \delta u_{i}\right|_{y} \mathrm{~d} x+\left.\lambda_{y}^{i} \int_{-1}^{+1} \delta u_{i}\right|_{y} \mathrm{~d} x\right],
$$

where periodicity in $x$ was used. Since $\psi_{i}=$ const at $y=0,1$, we can choose $\lambda_{y}^{i}=-\left.\psi_{i}\right|_{y}$ thus implying the variational derivative $\delta \widehat{H} / \delta q_{i}=-\psi_{i}$. Thus, the Poisson operator in the Hamiltonian formulation of (2.1), namely

$$
\frac{\partial q_{i}}{\partial t}=J \frac{\delta \widehat{H}}{\delta q_{i}},
$$

is given by $J=-\partial\left(q_{i}, \cdot\right)$ with $\partial(\alpha, \beta)=\alpha_{x} \beta_{y}-\alpha_{y} \beta_{x}$ being the usual Jacobian. This also allows one to formulate the problem in terms of a Poisson bracket:

$$
\partial_{t} q_{i}=\left\{q_{i}, \widehat{H}\right\},
$$

with the bracket defined according to

$$
\{F, \widehat{H}\}=\left(\frac{\delta F}{\delta q_{i}}, J \frac{\partial H}{\partial q_{i}}\right)=-\int_{\mathbf{D}} \frac{\delta F}{\delta q_{i}} \partial\left(q_{i}, \frac{\partial H}{\partial q_{i}}\right) \mathrm{d} \mathbf{x} .
$$

It is interesting to notice that the analysis shows the impossibility of reduction of (3.7) to the standard Lie-Poisson form as opposed to the case treated by MCINTYRE and SHEPHERD [59] in view of the specific boundary conditions.

Evidently, (3.5) is a noncanonical Hamiltonian system and the operator $J$ is not invertible. Therefore, the fixed point

$$
\psi_{i}=\psi_{i}^{e}: \frac{\partial q_{i}}{\partial t}=J \frac{\delta \widehat{H}}{\delta q_{i}}=0,
$$

only implies that $\delta \widehat{H} / \delta q_{i}$ lies in the null space of $J$. Hence at $\psi_{i}=\psi_{i}^{e}$ we expect that

$$
\frac{\delta \widehat{H}}{\delta q_{i}}=-\frac{\delta \mathcal{C}_{i}}{\delta q_{i}},
$$

for some Casimir(s) $\mathcal{C}_{i}$ - the null eigenvector(s) of $J$ :

$$
J \frac{\delta \mathcal{C}_{i}}{\delta q_{i}}=0 \Rightarrow \frac{\delta \mathcal{C}_{i}}{\delta q_{i}}=C_{i}^{\prime}\left(q_{i}\right) \Rightarrow \mathcal{C}_{i}=\int_{\mathbf{D}} C_{i}\left(q_{i}\right) \mathrm{d} \mathbf{x},
$$

where $C_{i}\left(q_{i}\right)$ is an arbitrary function. These Casimirs are a direct consequence of the conservation of potential vorticity along fluid particle trajectories in the 
conservative case of (2.1). In particular, this implies conservation of the $L^{p}$ norms of $q_{i}$ including $p=\infty$. The existence of $\mathcal{C}_{i}$ implies that the dynamics is taking place on a hypersurface defined by all conservation laws, that is, bulk and boundary circulations and Casimirs. It must be noted that the Casimir $\mathcal{C}_{i}$ is determined by the sufficient condition (3.10), but there is no guarantee that there are no other Casimirs. This has some implications for the stability analysis: the more complete the set of Casimirs, the narrower the space of admissible dynamics, but the sharper the stability boundary estimates.

\section{Linear spectral stability analysis}

Here we review the linear spectral stability of the basic state, $\psi_{i}^{e}=-U_{i} y$. The linear part of the operator equation (2.10) is

$$
\begin{aligned}
\partial_{t} M \phi_{i}+L \phi_{i}:= & \left(\partial_{t}+U_{i} \partial_{x}\right)\left[\nabla^{2} \phi_{i}+(-1)^{i} F\left(\phi_{1}-\phi_{2}\right)\right] \\
& +\partial_{x} \phi_{i}\left[\beta-(-1)^{i} F\left(U_{1}-U_{2}\right)\right]+r \nabla^{2} \phi_{i}=0 .
\end{aligned}
$$

Our goal here is to perform a linear spectral stability analysis of this system which amounts to its reduction to the generalized eigenvalue problem via $\phi_{i} \rightarrow \mathrm{e}^{\sigma t} \widehat{\phi}_{i}$ :

$$
\sigma M \phi_{i}+L \phi_{i}=0
$$

Clearly, the operator $M$ is of elliptic type, as shown in the introduction to Section 6, and invertible, while the operator $L$ is unbounded. As will be shown below, the spectrum of (4.2) is discrete. Let us decompose the eigensolution into Fourier modes:

$$
\widehat{\phi}_{i}=\sum_{m=1}^{\infty} \sum_{n=-\infty}^{+\infty} \widetilde{\phi}_{m n}^{i} \mathrm{e}^{i \pi n x} \sin \pi m y,
$$

which form a complete basis for this problem. Here we take into account periodicity in $x$, anti-symmetry in $y$ and homogeneous boundary conditions for $\phi_{i}$ at $y=0,1$. Omitting indexes $m, n$ and using the notation $a^{2}=\pi^{2}\left[n^{2}+m^{2}\right]$, this produces an algebraic system of equations:

$$
\begin{aligned}
(\sigma & \left.+i \pi n U_{j}\right)\left[(-1)^{j} F\left(\widetilde{\phi}^{1}-\widetilde{\phi}^{2}\right)-a^{2} \widetilde{\phi}^{j}\right] \\
& +i \pi n \widetilde{\phi}^{j}\left[\beta-(-1)^{j} F\left(U_{1}-U_{2}\right)\right]=r a^{2} \widetilde{\phi}^{j}, \quad j=1,2,
\end{aligned}
$$

which has a nontrivial solution only if determinant of the above system vanishes. The resulting eigenvalues are computed to be

$$
\begin{aligned}
\sigma_{1,2} & =-i \frac{\pi n\left(U_{1}+U_{2}\right)}{2}+\frac{a^{2}+F}{a^{2}+2 F}\left(i \frac{\beta \pi n}{a^{2}}-r\right) \\
\mp & \frac{\sqrt{\pi^{2} n^{2} a^{4}\left(4 F^{2}-a^{4}\right)\left(U_{1}-U_{2}\right)^{2}-4 F^{2}\left(\beta \pi n+i r a^{2}\right)^{2}}}{2 a^{2}\left(a^{2}+2 F\right)} ;
\end{aligned}
$$


that is, the spectrum is discrete and consists of two disjoint sets $\sigma_{+}$and $\sigma_{-}$of a countable number of eigenvalues of finite multiplicity with $\operatorname{Re} \sigma_{+}>0$ and $\operatorname{Re} \sigma_{-}<0$ and there are no points of accumulation. Note that the algebraic expressions (4.4) are of the form $\sigma_{1,2}=A+i B \mp \sqrt{C+i D}$. The Hamiltonian case is reproduced by taking $r=0$ in this expression. The marginal (neutral) stability is defined by $\operatorname{Re} \sigma=0$. While the conservative case $(r=0)$ is straightforward to analyze, the dissipative case involves some algebra. First of all, the equation $\sqrt{C+i D}=q_{R}+i q_{I}$ has two solutions:

$$
q_{R}^{1,2}=\sqrt{\frac{C \pm \sqrt{C^{2}+D^{2}}}{2}}, \quad q_{I}^{1,2}=\frac{D}{2 q_{R}} .
$$

Therefore, when analyzing the real part of $\sigma$, one has to consider four equations, $A \mp q_{R}^{1,2}=0$, which yield the same solution for the critical value $U_{c}=U_{1}-U_{2}$ :

$$
\frac{U_{c} F}{2 \beta}=\frac{\sqrt{F}}{a}\left[1+\frac{a^{2}}{F}\right]^{-1}\left[2-\frac{a^{2}}{F}\right]^{-1 / 2}\left[1+\left(\frac{a^{2} r}{\beta \pi n}\right)^{2}\left(1+\frac{a^{2}}{F}\right)^{2}\right]^{1 / 2},
$$

marked by dots on the dashed curve in Fig. 5 in the case of vanishing dissipation. The dashed curve itself corresponds to the continuous spectrum case when the dimension of the channel in the $x$-direction is infinite. In general, the longer the channel, the more dense the distribution of wavenumbers along the marginal stability curve. In the case of a finite channel the actual locations of wavenumbers are also determined by the value of the parameter $F$ : thus, without loss we can consider the critical bifurcation parameters corresponding to extrema of the continuous curve. The same logic applies to the neutral stability curve in the conservative case. The last step is to analyze stability below and above the marginal stability curve, which is easy to compute analytically by expanding around the marginal stability curve for small dissipation, $r \ll 1$, and small distance from $U_{c}$, that is, $U_{1}-U_{2}=U_{c}+s$ :

$$
\begin{aligned}
& \operatorname{Re} \sigma_{1}=\frac{a^{3}\left(a^{2}+F\right)^{2} \sqrt{2 F-a^{2}}}{2 F^{3} \beta} s r+\cdots, \\
& \operatorname{Re} \sigma_{2}=-2 \frac{a^{2}+F}{a^{2}+2 F}-\frac{a^{3}\left(a^{2}+F\right)^{2} \sqrt{2 F-a^{2}}}{2 F^{3} \beta} s r+\cdots,
\end{aligned}
$$

so that for $2 F>a^{2}$ we have linear spectral stability below the marginal stability curve and linear spectral instability above it — the shaded region in Fig. 5.

More specifically, in the Hamiltonian case the eigenvalues are obtained by setting $r=0$ in (4.4), from which one can observe that $\operatorname{Im} \sigma \sim \pi n$. Since both $\pi n$ and $-\pi n$ are legitimate wavenumbers, then we have four eigenvalues symmetrically located around the origin of the complex plane. Thus, the only spectrally stable configuration corresponds to $\operatorname{Re} \sigma=0$, which defines a region of neutral stability,

$$
\frac{U_{c} F}{2 \beta} \leqq\left(\frac{a^{2}}{F}\right)^{-1}\left(4-\frac{a^{4}}{F^{2}}\right)^{-1 / 2}, \quad U_{c}=\left|U_{1}-U_{2}\right|,
$$




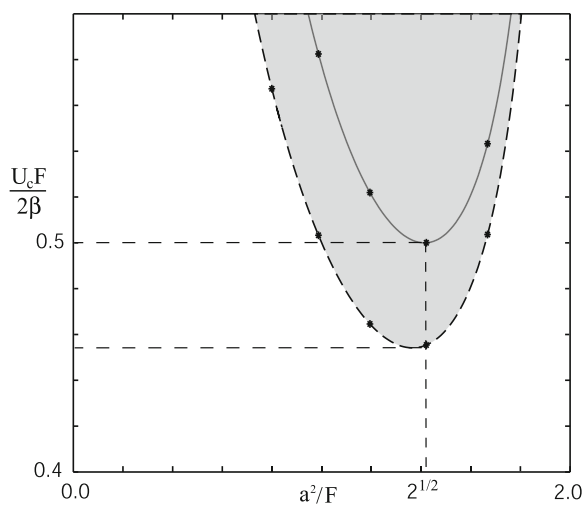

Fig. 5. Neutral stability curve (solid line) in the Hamiltonian case, $r=0$, and marginal stability curve (dashed line) in the case of vanishing dissipation, $r \rightarrow 0$. The whole shaded region corresponds to spectral instability for $r \rightarrow 0$

the envelope of which has an extremum for $a^{2} / F=\sqrt{2}$ with minimal magnitude of the bifurcation parameter $U_{c} F / 2 \beta=1 / 2$, that is, below this value the system is spectrally stable, while above it there is a certain range of unstable wavenumbers embraced by the neutral stability curve.

The dissipative case $(r \rightarrow+0)$ has different implications for the stability characteristics: the marginal stability curve defined by (4.6) separates the stable from the unstable region (shaded in Fig. 5). The value of this bifurcation parameter at the extremum value of wavenumber $a^{2} / F=\sqrt{2}$ in the Hamiltonian case is $U_{c} F / 2 \beta=[2(1+\sqrt{2})]^{-1 / 2}$, which is below the value of the critical parameter $\frac{1}{2}$ in the Hamiltonian case. Since the Hamiltonian neutral curve is exactly inside this region of instability, one can expect the phenomenon of dissipationinduced instability. At this stage, only in the linear spectral instability sense can one conclude that the introduction of dissipation destroys the stability of solutions for $U_{c} F / 2 \beta \in\left[2^{-1 / 2}(1+\sqrt{2})^{-1 / 2}, 1 / 2\right]$. While this is the counterintuitive consequence of the dissipation effect, an increase of the dissipation parameter $r$ enlarges the region of stability as can be inferred from (4.6) and which agrees with the intuition.

Lastly, we note that the linear solutions corresponding to wavenumbers satisfying $a^{2}>2 F$ have zero growth rate in the conservative case, while in the dissipative case the real part of the leading eigenvalue is always negative in that region.

\section{Nonlinear stability analysis}

The main objective of this section is to demonstrate the fact of dissipationinduced instability in (2.1) just from the positions of stability analysis; the existence and regularity of solutions will be studied in Section 6. First, in Section 5.1, we prove nonlinear stability in the conservative (Hamiltonian) case below the solid 
curve in Fig. 5. Next, in Section 5.2, we prove nonlinear instability above the dashed curve in Fig. 5, that is, in the dissipative case in the shaded region of Fig. 5, corresponding to the inequality $U_{c} F / 2 \beta>[2(1+\sqrt{2})]^{-1 / 2}$, which we know from the spectral linear stability analysis in Section 4 . The proof of instability in the Hamiltonian case in Section 5.1 is based on the ideas of ARNOLD [5,6], while the proof in the dissipative case in Section 5.2 is accomplished using semigroup theory and following the ideas of Yudovich [88], Henry [39] and FriedLander et al. [28].

\subsection{The Hamiltonian case: Arnold's method}

While our goal is to study the equilibrium of the basic state $\psi_{i}^{e}=-U_{i} y$, the analysis below will be performed for a more general equilibrium state and at the end will be applied to this particular choice of $\psi_{i}^{e}$. From the preceding linear stability analysis we know that under the conditions (4.8) the system is spectrally stable, which is not sufficient to assert linear and nonlinear stability. The purpose of this section is to prove nonlinear stability following ideas of ARNOLD [5,6] who extended the Lyapunov direct method to infinite-dimensional systems and fluid dynamics, in particular. For introductory discussions of Arnold's method, the reader may refer to Holm et al. [41], ShePherd [80] and SALmon [78].

Let us call the augmented Hamiltonian (that is, the Hamiltonian constrained by Casimir and boundary circulations) the expression

$$
H_{c}=\widehat{H}+\varrho_{1}+\bigodot_{2} \text {, }
$$

where $\widehat{H}$ is given by (3.4) and $\mathcal{C}_{i}$ by (3.10). Since the equilibrium should be an extremum point of the hypersurface restricted to Hamiltonian, boundary circulations, and Casimirs, it is defined by vanishing the first variation $\delta H_{c}=\left.\frac{d}{d \epsilon}\right|_{\epsilon=0}$ $H_{c}(\mathbf{q}+\epsilon \delta \mathbf{q})=0$, which is given by

$$
\begin{aligned}
\delta H_{c}= & \iint_{\mathbf{D}} F\left(\delta \psi_{1}-\delta \psi_{2}\right)\left\{\left(\psi_{1}-\psi_{2}\right)+(-1)^{i} C_{i}^{\prime}\left(q_{i}\right)\right\} \mathrm{d} \mathbf{x} \\
& +\iint_{\mathbf{D}} \delta \nabla^{2} \psi_{i}\left(C_{i}^{\prime}\left(q_{i}\right)-\psi_{i}\right) \mathrm{d} \mathbf{x}+\sum_{y=0,1}\left(\psi_{i}(y)+\lambda_{y}^{i}\right) \delta \Gamma_{i}(y),
\end{aligned}
$$

where, as usual, summation over $i$ is assumed and primes denote derivatives with respect to $q_{i}$. In deriving (5.2) we utilized the periodicity of the solution in $x$. Equation (5.2) allows one to determine the form of the equilibrium solution. First note that the equilibrium solutions of (2.1) in the Hamiltonian case $r=0$ are $\mathbf{v}_{i} \cdot \nabla q_{i}=0$, that is, $\nabla \psi_{i}^{e}$ and $\nabla q_{i}$ should be collinear. Sufficient condition for this to happen is the functional relationship $\psi_{i}=F_{i}\left(q_{i}\right)$ for some real-valued functions $F_{i}$. Next one can observe from (5.2) that $\delta H_{c}=0$ only if we have functional dependence of the potential vorticity $q_{i}$ and the stream-function $\psi_{i}$ :

$$
\lambda_{y}^{i}=-\psi_{i}(y) \text { for } y=0,1 ; \quad C_{i}^{\prime}\left(q_{i}\right)=\psi_{i},
$$


that is $F_{i}=C_{i}^{\prime}$, where $C_{i}\left(q_{i}\right)$ is an arbitrary function, as we noted in Section 3. Compared to the conclusion $\psi_{i}=F_{i}\left(q_{i}\right)$ from (2.1), (5.3) makes it more restrictive by demanding $q_{i}$ be an invertible, hence monotone, function of $\psi_{i}$. To evaluate the effect of a perturbation in initial conditions we shall determine the second variation, which will also suggest a strategy for constructing a norm in which the disturbance remains bounded. Keeping terms of the second order and taking into account that the first variation vanishes, we find

$$
\delta^{2} H_{c}=\frac{1}{2} \iint_{\mathbf{D}}\left\{F\left(\delta \psi_{1}-\delta \psi_{2}\right)^{2}+\nabla \delta \psi_{i} \cdot \nabla \delta \psi_{i}\right\} \mathrm{d} \mathbf{x}+\frac{1}{2} \iint_{\mathbf{D}} C_{i}^{\prime \prime}\left(q_{i}\right) \delta q_{i}^{2} \mathrm{~d} \mathbf{x} .
$$

Apparently, the sufficient condition for the second variation to be (strongly) positive definite, that is $\delta^{2} H_{c}>0$, for any nonzero $\delta q_{i}$ is $C_{i}^{\prime \prime}\left(q_{i}\right)=\psi_{i}^{\prime}>0$, which yields a formal stability criteria. While the latter is neither necessary nor sufficient for nonlinear stability in an infinite-dimensional case, the proof of nonlinear stability in the Lyapunov sense will demonstrate that, in our case, positive definiteness of the second variation of the generalized Hamiltonian $\delta^{2} H_{c}>0$ together with convexity estimates are sufficient for nonlinear stability. To prove nonlinear stability we need to show that $\delta^{2} H_{c}$ remains bounded in the appropriate norm, which is constructed below with the help of convexity estimates.

First, let us construct two quadratic forms $Q_{1}$ and $Q_{2}$ such that the following convexity inequalities hold

$$
\begin{aligned}
& Q_{1}(\delta \mathbf{q}) \leqq H\left(\mathbf{q}^{e}+\delta \mathbf{q}\right)-H\left(\mathbf{q}^{e}\right)-\delta H\left(\mathbf{q}^{e}\right), \\
& Q_{2}(\delta \mathbf{q}) \leqq C\left(\mathbf{q}^{e}+\delta \mathbf{q}\right)-C\left(\mathbf{q}^{e}\right)-\delta C\left(\mathbf{q}^{e}\right),
\end{aligned}
$$

and where $Q_{1}(\delta \mathbf{q})+Q_{2}(\delta \mathbf{q})>0$ for all nonzero $\delta \mathbf{q}$. We can choose

$$
\begin{aligned}
& Q_{1}(\delta \mathbf{q})=\frac{1}{2} \iint_{\mathbf{D}}\left\{F\left(\delta \psi_{1}-\delta \psi_{2}\right)^{2}+\nabla \delta \psi_{i} \cdot \nabla \delta \psi_{i}\right\} \mathrm{d} \mathbf{x}, \\
& Q_{2}(\delta \mathbf{q})=\frac{c_{2}}{2} \iint_{\mathbf{D}} \delta q_{i} \delta q_{i} \mathrm{~d} \mathbf{x}, \quad c_{2} \leqq C_{i}^{\prime \prime}\left(q_{i}^{e}\right)=\psi_{i}^{\prime}\left(q_{i}^{e}\right),
\end{aligned}
$$

and then assign $Q_{1}(\delta \mathbf{q})+Q_{2}(\delta \mathbf{q})=\|\delta \mathbf{q}\|^{2}$ as the measure of a disturbance activity $\delta$ q. It suffices, though perhaps not necessary, to require the condition $c_{2}>0$. While positive definiteness and homogeneity of $\|\delta \mathbf{q}\|$ are easy to demonstrate, the triangle inequality, $\left\|\delta \mathbf{q}^{1}+\delta \mathbf{q}^{2}\right\| \leqq\left\|\delta \mathbf{q}^{1}\right\|+\left\|\delta \mathbf{q}^{2}\right\|$, follows from the use of Minkowski's inequality and the observation that the decomposition $\delta q_{i}=\delta q_{i}^{1}+\delta q_{i}^{2}$ is equivalent to $\delta \psi_{i}=\delta \psi_{i}^{1}+\delta \psi_{i}^{2}$.

It can be shown that $H_{c}$ is continuous at $\mathbf{q}^{e}$ in the norm $\|\delta \mathbf{q}\|$ since the following sufficient conditions are satisfied: there are constants $\widetilde{C_{1}}, \widetilde{C_{2}}$ such that

$$
\begin{aligned}
& \widehat{H}\left(\mathbf{q}^{e}+\delta \mathbf{q}\right)-\widehat{H}\left(\mathbf{q}^{e}\right)-\widehat{H}^{\prime}\left(\mathbf{q}^{e}\right) \delta \mathbf{q} \leqq \widetilde{C_{1}}\|\delta \mathbf{q}\|^{2}, \\
& \mathcal{C}\left(\mathbf{q}^{e}+\delta \mathbf{q}\right)-\mathcal{C}\left(\mathbf{q}^{e}\right)-\mathcal{C}^{\prime}\left(\mathbf{q}^{e}\right) \delta \mathbf{q} \leqq \widetilde{C_{2}}\|\delta \mathbf{q}\|^{2},
\end{aligned}
$$

which in our case should satisfy $\infty>\widetilde{C_{2}} \geqq \psi_{i}^{\prime}\left(q_{i}^{e}\right) \geqq c_{2}>0, i=1,2$. The latter follows from

$$
\frac{1}{2} \iint_{\mathbf{D}} C_{i}^{\prime \prime}\left(q_{i}\right) \delta q_{i}^{2} \mathrm{~d} \mathbf{x} \leqq \frac{\widetilde{C_{2}}}{2} \iint_{\mathbf{D}} \delta q_{i}^{2} \mathrm{~d} \mathbf{x} \leqq \widetilde{C_{2}}\|\delta \mathbf{q}\|^{2}, \quad \widetilde{C_{2}} \geqq C_{i}^{\prime \prime}\left(q_{i}\right),
$$


The nonlinear stability results can be formulated in the following theorem, cf. also [41]. Note that, similar to the Lyapunov direct method, this theorem would be "conditional" without the existence of global classical solutions of (2.1). The latter, however, is established in Section 6.3, and hence makes this stability result unconditional.

Theorem 2. Global classical solutions of the problem (2.1) exist. If the convexity estimates (5.4) hold, which define the norm $\|\cdot\|$, and if $H_{c}$ is continuous in this norm, as guaranteed by (5.6), then the stability estimate

$$
\|\delta \mathbf{q}(t)\|^{2}=Q_{1}(\delta \mathbf{q})+Q_{2}(\delta \mathbf{q}) \leqq\left(\widetilde{C_{1}}+\widetilde{C_{2}}\right)\|\delta \mathbf{q}(0)\|^{2},
$$

holds for all nonzero $\delta \mathbf{q}$; that is, the equilibrium solution $\mathbf{q}^{e}$ is Lyapunov stable.

Proof. In Section 6.3, Theorem 6, we construct a space $X$ in which global classical solutions are shown to exist.

To establish (5.8), first note that $H_{c}$ is a constant of motion. Indeed, since $H_{c}=\widehat{H}+\mathcal{C}_{1}+\mathcal{C}_{2}$ from Equation (5.1) and $\widehat{H}=H+\sum_{y=0,1} \lambda_{y}^{i} \Gamma_{i}(y)$ from Equation (3.4), then

$$
\frac{\mathrm{d} \widehat{H}}{\mathrm{~d} t}=\frac{\mathrm{d} H}{\mathrm{~d} t}+\sum_{y=0,1} \lambda_{y}^{i} \frac{\mathrm{d} \Gamma_{i}(y)}{\mathrm{d} t}=0,
$$

where $\mathrm{d} H / \mathrm{d} t=0$ by (3.3), since $r=0$ when there is no dissipation, and $\mathrm{d} \Gamma_{i}(y) / \mathrm{d} t=0$ by (2.3), and finally by (3.10):

$$
\frac{\mathrm{d}_{i}}{\mathrm{~d} t}=\int_{\mathbf{D}} \frac{\mathrm{d} C_{i}\left(q_{i}\right)}{\mathrm{d} t} \mathrm{~d} \mathbf{x}=\int_{\mathbf{D}} C_{i}^{\prime} \frac{\mathrm{d} q_{i}}{\mathrm{~d} t} \mathrm{~d} \mathbf{x}=\int_{\mathbf{D}} C_{i}^{\prime}\left(\frac{\partial q_{i}}{\partial t}+\mathbf{v}_{i} \cdot \nabla q_{i}\right) \mathrm{d} \mathbf{x}=0,
$$

since, as we mentioned in Section 3, the Casimirs are a direct consequence of the conservation of potential vorticity along fluid particle trajectories in the conservative case of (2.1).

Based on the fact that $H_{c}$ is a constant of motion and from inequalities (5.4) we observe that

$$
\begin{aligned}
Q_{1}(\delta \mathbf{q})+Q_{2}(\delta \mathbf{q}) & \leqq H_{c}\left(\mathbf{q}^{e}+\delta \mathbf{q}\right)-H_{c}\left(\mathbf{q}^{e}\right)-\delta H_{c}\left(\mathbf{q}^{e}\right) \\
& =H_{c}\left(\mathbf{q}^{e}+\delta \mathbf{q}\right)-H_{c}\left(\mathbf{q}^{e}\right)=H_{c}(\mathbf{q}(0))-H_{c}\left(\mathbf{q}^{e}\right) .
\end{aligned}
$$

Thus,

$$
\|\delta \mathbf{q}(t)\|^{2}=Q_{1}(\delta \mathbf{q})+Q_{2}(\delta \mathbf{q}) \leqq H_{c}(\mathbf{q}(0))-H_{c}\left(\mathbf{q}^{e}\right) .
$$

Now, we can prove the Lyapunov stability, that is, given $\epsilon>0$, there is a $\delta>0$ such that $\mathbf{q}(t)$ never leaves the $\epsilon$-ball about $\mathbf{q}^{e}$ if it starts in the $\delta$-ball. Since the continuity of $H_{c}$ implies that if $\left\|\mathbf{q}(0)-\mathbf{q}^{e}\right\|<\delta$ then $\left\|H_{c}(\mathbf{q}(0))-H_{c}\left(\mathbf{q}^{e}\right)\right\|<\epsilon$, from (5.9) it follows

$$
\|\delta \mathbf{q}(t)\|^{2} \leqq\left\|H_{c}(\mathbf{q}(0))-H_{c}\left(\mathbf{q}^{e}\right)\right\|<\epsilon \quad \text { if } \quad\|\delta \mathbf{q}(0)\|<\delta .
$$

Using (5.6),

$$
H_{c}\left(\mathbf{q}^{e}+\delta \mathbf{q}(0)\right)-H_{c}\left(\mathbf{q}^{e}\right)=H_{c}\left(\mathbf{q}^{e}+\delta \mathbf{q}\right)-H_{c}\left(\mathbf{q}^{e}\right) \leqq\left(\widetilde{C_{1}}+\widetilde{C_{2}}\right)\|\delta \mathbf{q}(0)\|^{2},
$$

which proves (5.8). 
Note that if one could establish the existence of solutions in a larger space than $X$ in which $Q_{1}$ and $Q_{2}$ are still well-defined, then the same argument as in the proof of Theorem 2 would apply. Hence, the above theorem probably applies to a wider class of solutions.

Now, we are in a position to apply the above results to our particular basic state $\psi_{i}^{e}=-U_{i} y$, which is an equilibrium solution. In order to compute $\psi_{i}^{\prime}\left(q_{i}^{e}\right)$, observe that

$$
\nabla \psi_{i}^{e}=\psi_{i}^{\prime}\left(q_{i}^{e}\right) \nabla q_{i}^{e}(\text { no summation over } i)
$$

that is, $\nabla \psi_{i}^{e}$ and $\nabla q_{i}^{e}$ are collinear. This, together with the definitions of $\psi_{i}^{e}$ and $q_{i}^{e}$, furnishes

$$
\psi_{i}^{\prime}\left(q_{i}^{e}\right)=\frac{U_{i}}{(-1)^{i} F\left(U_{1}-U_{2}\right)-\beta} .
$$

Note that while we require $\infty>\widetilde{C_{2}} \geqq \psi_{i}^{\prime}\left(q_{i}^{e}\right) \geqq c_{2}>0$, we are free to choose $c_{2}$ as small as we want, and $\widetilde{C_{2}}$ as large as we wish (as in ARNOLD [6]), and therefore the solution is Lyapunov stable in the limiting situation:

$$
\infty>\psi_{i}^{\prime}\left(q_{i}^{e}\right)>0 \Rightarrow-\frac{1}{2}<\frac{U_{c} F}{2 \beta}<\frac{1}{2}, \quad U_{c}=\left|U_{1}-U_{2}\right| .
$$

This proves that the solution is nonlinearly stable in the Hamiltonian case for $U_{c} F / 2 \beta<1 / 2$, that is, below the solid curve in Fig. 5 .

\subsection{The dissipative case}

It is tempting to draw the conclusion that the solution in the dissipative case is unstable above the marginal stability curve defined by (4.6) and stable below it. However, there is no general analog of the Lyapunov theorem on deducing linear and nonlinear instability based on spectral information for infinite-dimensional systems, cf. LuO et al. [56]. When one studies stability of a solution $\phi$ of an infinite-dimensional system, the problem often reduces to the question of stability of a solution in the Banach space $X$ of the nonlinear evolution equation (2.12) with stationary principal part $\mathcal{A} \phi$, that is, when the linear operator $\mathcal{A}: X \rightarrow X$ is time-independent.

In this context, the main idea of the proof of the instability in the dissipative case reduces to considering the original PDE problem as an infinite-dimensional evolutionary ODE (2.12) in some suitable Banach space $X$. Then, one approach to proving instability is to use semigroup theory together with the geometric theory of semi-linear parabolic equations following the original ideas of KREIN [22], Yudovich [88], and Henry [39]. Using this method we shall obtain the main result of this section, namely

Theorem 3. Let $s \geqq 2 / q$ with $2<q<\infty$. If $f^{10} U_{c} F / 2 \beta>2^{-1 / 2}(1+\sqrt{2})^{-1 / 2}$, then the equilibrium solution of (2.1) is Lyapunov unstable in $W^{s, q}$.

\footnotetext{
10 This choice of the bifurcation parameter in (2.1) implies that the spectrum of the linear operator $\mathcal{A}$ over Sobolev space contains points in the right half of the complex plane.
} 
The choice of function spaces in Theorem 3 (for the definitions of spaces refer back to Section 2.3) is dictated by the fact that we have a local existence result for classical solutions in Hölder spaces $C^{k, \lambda}$, while the nature of the problem allows us to perform the stability analysis only in a weaker topology, namely in the Sobolev spaces $W^{s, p}$. The latter, however, suffices since the natural inclusion $C^{k, \lambda} \rightarrow W^{s, p}$ defines a bounded operator and thus the norms of functions obey

$$
\|u\|_{W^{s, p}} \leqq C\|u\|_{C^{k, \lambda}},
$$

for example for $k \geqq s$ and $0<\lambda<1$. In fact, one has the compactness of the embedding $C^{k, \lambda} \hookrightarrow W^{s, p}$. The above relation between the norms implies that an instability in $W^{s, p}$ guarantees an instability in $C^{k, \lambda}$. Note, that the proof of stability in $W^{s, p}$ would only give stability in $C^{k, \lambda}$ for $s>\frac{n}{p}+k+\lambda$ by the Sobolev embedding theorem. In the analysis below, as usual, constant $C$ stands for a generic constant that may change from line to line.

5.2.1. Problem statement and a few definitions. The evolution system (2.1) for the perturbation $\phi$ in the Orr-Sommerfeld-like form (2.10) can be recast in the standard form (2.12) using the fact that the "mass" operator $M$ is invertible, which renders it suitable for the application of the infinite-dimensional dynamical systems theory. The invertibility of the operator $M$ in (2.10) can be seen using Green's function methods (see also the discussion in the introduction of Section 6 , where we demonstrate that $M$ is an elliptic operator): namely, consider

$$
M \mathbf{G}=\left(\begin{array}{cc}
-\nabla^{2}-F & F \\
F & -\nabla^{2}-F
\end{array}\right) \mathbf{G}=\boldsymbol{\delta}(\mathbf{x}-\xi),
$$

where $\mathbf{x}=(x, y)$ and $\boldsymbol{\xi}=(\xi, \eta)$. Writing $\mathbf{G}=\sum_{m, n}\left(\begin{array}{c}a_{m n}^{1} \\ a_{m n}^{2}\end{array}\right) \mathrm{e}^{i \pi n x} \sin \pi m y$, we find that

$$
\mathbf{G}(x, \xi, y, \eta)=-\sum_{m=1}^{\infty} \sum_{n=-\infty}^{+\infty}\left(\begin{array}{l}
1 \\
1
\end{array}\right) a^{-2} \mathrm{e}^{i \pi n(x-\xi)} \sin \pi m y \sin \pi m \eta,
$$

where $a^{2}=\pi^{2}\left(n^{2}+m^{2}\right) \neq 0$ for all integers $n, m$, which guarantees the invertibility of $M$. Thus, the operator $M^{-1}$ applied to a vector-function $\mathbf{f}$ is the smoothing operation given by $M^{-1} \mathbf{f}=\int_{-1}^{1} \int_{0}^{1} \mathbf{G}(x, \xi, y, \eta) \mathbf{f}(\xi, \eta) \mathrm{d} \xi \mathrm{d} \eta$.

Now we take advantage of the fact that operator $M$ in (2.10) is invertible to rewrite (2.10) in the standard form (2.12) for $\boldsymbol{\phi}=\left(\phi_{1}, \phi_{2}\right)$ :

$$
\frac{\partial \phi}{\partial t}=\mathcal{A} \boldsymbol{\phi}+\mathcal{N}(\boldsymbol{\phi}, \boldsymbol{\phi}), \quad \text { with } \mathcal{A}=-M^{-1} L, \mathcal{N}=M^{-1} N,
$$

where notation $\mathcal{N}(\boldsymbol{\phi}, \boldsymbol{\phi})$ signifies that the nonlinearity is "quadratic" in $\boldsymbol{\phi}$. Mild solutions, as introduced by Kato and FuJiTA [45], of this equation can be written and determined via Duhamel's formula:

$$
\boldsymbol{\phi}(t)=\boldsymbol{\phi}(0) \mathrm{e}^{\mathcal{A} t}+\int_{0}^{t} \mathrm{e}^{\mathcal{A}(t-\tau)} \mathcal{N}(\boldsymbol{\phi}(\tau), \boldsymbol{\phi}(\tau)) \mathrm{d} \tau,
$$


where the last integral will be denoted by $\mathcal{B}(t)=\int_{0}^{t} \mathrm{e}^{\mathcal{A}(t-\tau)} \mathcal{N}(\boldsymbol{\phi}(\tau), \boldsymbol{\phi}(\tau)) \mathrm{d} \tau$, for convenience in the subsequent analysis. However, our goal here is not to construct mild solutions, say via fixed point iteration, but to prove Lyapunov instability of the equilibrium solution (2.13) in the weak topology of $W^{s, p}$ using (5.17). The latter formula also highlights the importance of both the linear and the nonlinear terms for stability analysis in infinite dimensions: indeed, the nonlinear term $\mathcal{N}$ is unbounded in $W^{s, p}$ in view of the presence of unbounded operations of differentiation in it, cf. (2.11c), which can be compensated only by the smoothing effect of the semigroup $\mathrm{e}^{\mathcal{A}(t-\tau)}$. Thus, naturally, for the stability analysis we will need to study not only the properties of the linear operator (cf. Section 5.2.2), but also to determine bounds on the nonlinear terms (cf. Section 5.2.3), which is done with the help of the fractional powers of the linear operator $\mathcal{A}$-an idea which goes back to KREIN [50] (see also Yudovich [88]).

For the purpose of both immediate and further discussions, let us introduce the basic definitions characterizing the linear operator $\mathcal{A}$. The resolvent set $\rho(\mathcal{A})$ is the set of all points $\lambda$ in the complex plane $C$ where the resolvent $R_{\lambda}=(\mathcal{A}-\lambda I)^{-1}$ is defined. Then, the complement of $\rho(\mathcal{A})$, the spectrum of $\mathcal{A}$, is denoted $\sigma(\mathcal{A})$.

With Definition 4 of instability, in the following lemma we demonstrate that the local existence result in the stronger topology of $C^{k, \lambda}$ is enough in order to prove Lyapunov instability of a solution in that space and its existence until instability happens, that is, strong Lyapunov instability, given the proof of Lyapunov instability in the weaker topology of $W^{s, p}$. In fact, the following instability lemma is of general interest, but we state it for the case of the $C^{k, \lambda}$ and $W^{s, p}$ topologies to be concrete.

Lemma 1. Suppose $\boldsymbol{\phi}^{e}$ is an equilibrium solution and a local existence theory ${ }^{11}$ applies to solutions in $C^{k, \lambda}, 0<\lambda<1$, in some neighborhood of $\boldsymbol{\phi}^{e}$. Then, if $\boldsymbol{\phi}^{e}$ is Lyapunov unstable in $W^{s, p}$ with $s \leqq k$, then it is strongly Lyapunov unstable in $C^{k, \lambda}$.

Proof. Let us first make two easy preparatory statements:

(A) Let $B$ be a ball of radius $\mu$ in $C^{k, \lambda}$. If a solution $\phi$ exists locally and a priori stays in $B$; that is, $\|\phi\|_{C^{k, \lambda}} \leqq \mu$, then it exists for all time by our assumption of local continuation in time. This also means that a solution would exist for all time in $W^{s, p}$ in a ball of radius $\mu C$, where $C$ is the constant from (5.14).

(B) If instability takes place in $W^{s, p}$ according to Definition 4, then there exists a ball of radius $\epsilon C$ and a moment $t_{1}$ such that $\left\|\phi\left(t_{1}\right)\right\|_{W^{s, p}} \geqq \epsilon C$ and therefore the solution escapes not only from the ball of radius $\epsilon C$ in $W^{s, p}$, but also from the ball of radius $\epsilon$ in $C^{k, \lambda}$. Hence, the solution is unstable in $C^{k, \lambda}$ too.

Therefore, if a solution exists in $C^{k, \lambda}$ long enough so that (B) holds, then clearly strong Lyapunov instability in $C^{k, \lambda}$ is proved; that is what was demonstrated in the proof of Theorem 3. Next, let us fix the size $\epsilon$ of the ball $B$ in $C^{k, \lambda}$. If a solution exists long enough to escape from the ball $B$, then this implies strong Lyapunov

11 We take it for granted, which holds in our case, that the local existence theory contains the result that solutions with an a priori bound in $C^{k, \lambda}$ can be continued in time. 
instability in $C^{k, \lambda}$. If, however, a solution stays in the ball, then according to (A), it continues to exist, and one can apply (B) to prove instability. The above arguments indicate that even if the local existence result given in Theorem 5 gives the time of existence, which is less than the critical time of instability $t_{1}$, with the above argument one can see that the solution exists at least up to $t_{1}$.

5.2.2. Properties of the linear operator $\mathcal{A}$. Since the operator $\mathcal{A}$ is unbounded, we need to understand some of its properties in order to proceed with the stability analysis. The relevant properties of the operator $\mathcal{A}$ are given by the following lemma.

Lemma 2. The operator $\mathcal{A}$ is elliptic and in $W^{s, p}$ is the infinitesimal generator of an analytic semigroup.

From the solution of the linear eigenvalue problem, that is, the linear OrrSommerfeld equation (4.1), we know that the spectrum of the operator $\mathcal{A}$ is discrete and its eigenvalues $\sigma_{m n}$ given by (4.4), enumerated with $m \in \mathbb{Z}$ and $n \in \mathbb{N}^{+}$, have asymptotics $\sigma_{m n} \rightarrow(x \pm i y) n$ in the complex plane $\mathbb{C}$, for $|m|, n \gg 1$ and constant $x, y$. If we consider the case when the spectrum $\sigma(\mathcal{A})$ lies in the left half-plane, then it is clear that the asymptotes $(x \pm i y) n$ belong to the left half-plane. Also, we observe that for finite $m, n$ the imaginary part of each eigenvalue is finite. Therefore, regardless of the actual behavior of the eigenvalues for $m, n=O(1)$, a sector $S_{\delta}=\left\{\sigma_{m n} \in \mathbb{C}:\left|\arg \sigma_{m n}\right|<\frac{\pi}{2}+\delta\right\} \backslash\{0\}, 0<\delta \leqq \frac{\pi}{2}$ is contained in the resolvent set $\rho(\mathcal{A})$, that is, the spectrum $\sigma(\mathcal{A})$ lies within $\mathbb{C} \backslash S_{\delta}$ in the left half-plane, as in Fig. 6. While the derivation of the spectrum of $\mathcal{A}$ in Section 4 is purely formal, since the operator $\mathcal{A}$ is elliptic, the spectrum is exactly the same in $W^{s, p}$ and its eigenfunctions are in $C^{\infty}$ [31]. Next, since the spectrum of $\mathcal{A}$ is discrete, then for the resolvent and $\lambda \in \rho(\mathcal{A})$ we have

$$
\begin{aligned}
R_{\lambda}(\mathcal{A}, \lambda) \mathbf{x} & =(\mathcal{A}-\lambda)^{-1} \mathbf{x}=\sum_{m, n}\left(\sigma_{m n}-\lambda\right)^{-1} \xi_{m n} \boldsymbol{\phi}_{m n} \\
& \leqq \max _{m, n}\left(\sigma_{m n}-\lambda\right)^{-1} \sum_{m, n} \xi_{m n} \boldsymbol{\phi}_{m n}=\max _{m, n}\left(\sigma_{m n}-\lambda\right)^{-1} \mathbf{x}
\end{aligned}
$$

hence there exists $C$ such that

$$
\left\|(\mathcal{A}-\lambda)^{-1}\right\| \leqq C /|\lambda-a| .
$$

This proves that the operator $\mathcal{A}$ is sectorial, and therefore $(a)$ it is the infinitesimal generator of an analytic semigroup $\mathrm{e}^{\mathcal{A} t}$, cf. Theorem 1.3.4 of HeNRY [39], $(b)$ it possesses the spectral mapping property, and $(c)$ moreover:

$$
\mathrm{e}^{\mathcal{A} t}=\frac{1}{2 \pi i} \int_{\Gamma}(\lambda-\mathcal{A})^{-1} \mathrm{e}^{\lambda t} \mathrm{~d} \lambda,
$$

where $\Gamma$ is a contour in $\rho(\mathcal{A})$, as shown in Fig. 6. From the latter property it follows $[27,39,67]$ that if $\operatorname{Re} \sigma(\mathcal{A})<a<0$, then

$$
\left\|\mathcal{A}^{\alpha} \mathrm{e}^{\mathcal{A} t}\right\|_{W^{s, q} \rightarrow W^{s, q}} \leqq \frac{C}{t^{\alpha}} \mathrm{e}^{a t} .
$$




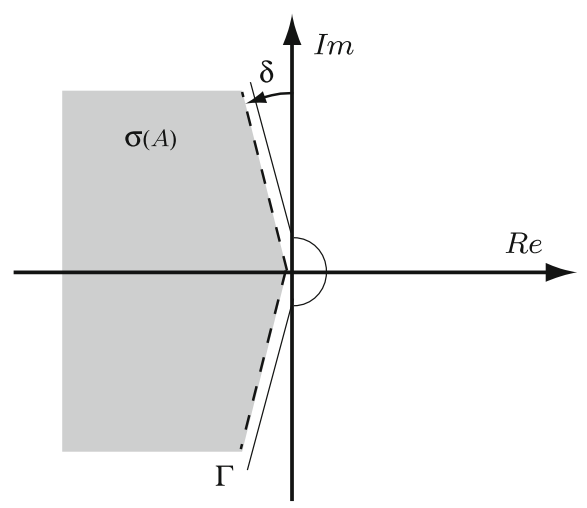

Fig. 6. On the definition of a sectorial operator

Again, because $\mathcal{A}$ is elliptic, as shown below, and its eigenfunctions and eigenvalues are the same in $W^{s, p}$, as say in $L^{p}$, the above statement can be made with regard to any Sobolev space. Also, because $\mathcal{A}$ generates an analytic semigroup, the spectral mapping theorem applies [27], that is $\sigma\left(\mathrm{e}^{\mathcal{A} t}\right)=\mathrm{e}^{t \sigma(\mathcal{A})}$.

Finally, it is required to establish that the operator $\mathcal{A}$ is elliptic, so that one can use the following Schauder-type inequality [66] for the elliptic operator $\mathcal{A}$ of order $m$ :

$$
\left\|\mathcal{A}^{-\alpha} f\right\|_{k, p} \leqq C\|f\|_{k-m \alpha, p} .
$$

The proof that $\mathcal{A}=-M^{-1} L$ is elliptic can be achieved by demonstrating that $M$ and $L$ are each elliptic. While the operator $M$ is clearly elliptic of the second order (see also the introduction to Section 6), the ellipticity of the operator $L$ can be obtained from the analysis of its principal part

$$
L^{\prime} \boldsymbol{\phi}=\left(\begin{array}{cc}
U_{1} \partial_{x} \nabla^{2} & 0 \\
0 & U_{2} \partial_{x} \nabla^{2}
\end{array}\right) \boldsymbol{\phi}
$$

Hence, its symbol is

$$
L^{\prime} \xi=\left(\begin{array}{cc}
U_{1} \xi\left(\xi^{2}+\eta^{2}\right) & 0 \\
0 & U_{2} \xi\left(\xi^{2}+\eta^{2}\right)
\end{array}\right) \xi
$$

that is, the characteristic equation gives $\left|L^{\prime} \xi\right|=U_{1} U_{2} \xi^{2}\left(\xi^{2}+\eta^{2}\right)^{2} \neq 0$ for $\xi, \eta \neq 0$, which implies that there are no real characteristics, and so the operator $L$ is elliptic of the order 3. Therefore, the operator $\mathcal{A}=-M^{-1} L$ is a first order elliptic operator.

5.2.3. Properties of the nonlinear operator $\mathcal{N}$. Before proving the instability in the dissipative case, that is, Theorem 3 , let us establish a useful relation between the Sobolev norms of vector functions and their tensor product. 
Lemma 3. Suppose that $\mathbf{f} \otimes \mathbf{f} \in W^{s, q}(\Omega)$ with $\Omega \subseteq \mathbb{R}^{n}$. Then there exists a constant $C(n, \operatorname{dim}(\mathbf{f}), s)$ such that

$$
\|\mathbf{f} \otimes \mathbf{f}\|_{W^{s, q}} \leqq C\|\mathbf{f}\|_{W^{s, 2 q}}^{2} .
$$

Proof. The proof is simple but lengthy, so we will just outline the main idea and demonstrate the result for a few simple cases. First, consider the case in which $f$ is a scalar. In the Sobolev space of order $s=0$, we have

$$
\left\|f^{2}\right\|_{0, q}^{2}=\left[\int_{\Omega}\left|f^{2}\right|^{q} \mathrm{~d} x\right]^{1 / q}=\left[\int_{\Omega}|f|^{2 q} \mathrm{~d} x\right]^{2 / 2 q}=\|f\|_{0,2 q}^{2} .
$$

For the Sobolev space of higher order one can use the Cauchy inequality, $a b \leqq a^{2} / 2+b^{2} / 2$; in particular, for $s=1$ :

$$
\begin{aligned}
\left\|f^{2}\right\|_{1, q}^{2} & =\left[\int_{\Omega}\left|f^{2}\right|^{q} \mathrm{~d} x+\int_{\Omega}\left|2 f f^{\prime}\right|^{q} \mathrm{~d} x\right]^{1 / q} \\
& \leqq\left[\int_{\Omega}|f|^{2 q} \mathrm{~d} x+\int_{\Omega}|f|^{2 q} \mathrm{~d} x+\int_{\Omega}\left|f^{\prime}\right|^{2 q} \mathrm{~d} x\right]^{2 / 2 q} \leqq C\|f\|_{1,2 q}^{2},
\end{aligned}
$$

where one can choose $C=2^{1 / q}$. Continuing the same reasoning, one will find that constant $C$ is a function of the order $s$ of the Sobolev space and the spatial dimension $n$, that is, $C=C(s, n)$.

Analogously, one can get a similar result when $\mathbf{f}$ is vector valued. For example, in the case of $s=0$, again using the Cauchy inequality,

$\|\mathbf{f} \otimes \mathbf{f}\|_{0, q}=\left[\sum_{i, j} \int\left|f_{i} f_{j}\right|^{q} \mathrm{~d} x\right]^{\frac{1}{q}} \leqq\left[\sum_{i} \int \operatorname{dim}^{q}(\mathbf{f})\left|f_{i}\right|^{2 q} \mathrm{~d} x\right]^{\frac{2}{2 q}}=C\|\mathbf{f}\|_{0,2 q}^{2}$,

where $C=\operatorname{dim}(\mathbf{f})$. The general case of arbitrary $s$ is proved similarly, which yields in general $C=C(n, \operatorname{dim}(\mathbf{f}), s)$.

As we will see in the next lemma, the above result is useful when establishing bounds and estimates on the nonlinear terms.

Lemma 4. The bilinear form $\mathcal{B}(t)$ in (5.17) grows at most like the square of the norm of $\boldsymbol{\phi}$ : if $\|\boldsymbol{\phi}(t)\|_{W^{s, q}} \leqq \delta \mathrm{e}^{\lambda t}$ for all $t \leqq T$, then there exists a constant $C$ such that

$$
\|B(t)\|_{W^{s, q}} \leqq C\left[\delta \mathrm{e}^{\lambda t}\right]^{2}, t \leqq T .
$$

Proof. Conceptually, the basic idea of the proof goes back to Yudovich [88], which is still the most efficient technique of proving the nonlinear estimates for stability analysis purposes, see, for example [28]. First, for convenience, we introduce the following operator $\mathcal{A}_{v}=\mathcal{A}-\lambda-v$ with $0<v<\lambda$ chosen so that the spectrum of this new operator lies entirely in the left half-plane. Then we can use the semigroup bounds (5.19), that is, $\left\|\mathcal{A}_{v}^{\alpha} \mathrm{e}^{\mathcal{A}_{v} t}\right\|_{W^{s, q} \rightarrow W^{s, q}} \leqq C t^{-\alpha}$, and the Schauder-type inequalities (5.20), that is $\left\|\mathcal{A}_{v}^{-\alpha} f\right\|_{k, p} \leqq C\|f\|_{k-m \alpha, p}$, as applied 
to the operator $\mathcal{A}_{v}$, which are valid for all $\alpha>0[27,39,67]$. With this understanding, we can get a bound on the nonlinear terms $\mathcal{B}(t)$, namely that the bilinear form $\mathcal{B}(t)$ grows at most like the square of the norm of $\phi$. First, note that for any $\alpha>0$, $\mathcal{A}_{v}^{\alpha} \mathrm{e}^{\mathcal{A}_{v} t}=\mathrm{e}^{\mathcal{A}_{v} t} \mathcal{A}_{v}^{\alpha}$ on $D\left(\mathcal{A}_{v}^{\alpha}\right)$, and thus we can write

$$
\begin{aligned}
\mathcal{B}(t) & =\int_{0}^{t} \mathrm{e}^{\mathcal{A}(t-\tau)} \mathcal{N}(\boldsymbol{\phi}(\tau), \boldsymbol{\phi}(\tau)) \mathrm{d} \tau \\
& =\int_{0}^{t} \mathrm{e}^{(\lambda+v)(t-\tau)} \mathcal{A}_{v}^{\alpha} \mathrm{e}^{\mathcal{A}_{v}(t-\tau)} \mathcal{A}_{v}^{-\alpha} \mathcal{N}(\boldsymbol{\phi}(\tau), \boldsymbol{\phi}(\tau)) \mathrm{d} \tau
\end{aligned}
$$

and its norm in $W^{s, q}$ with the use of the semigroup bound can be estimated as

$$
\|\mathcal{B}(t)\|_{W^{s, q}} \leqq \int_{0}^{t} \mathrm{e}^{(\lambda+v)(t-\tau)} \frac{1}{(t-\tau)^{\alpha}}\left\|\mathcal{A}_{\nu}^{-\alpha} \mathcal{N}(\boldsymbol{\phi}(\tau), \boldsymbol{\phi}(\tau))\right\|_{W^{s, q}} \mathrm{~d} \tau .
$$

Next, the estimate of $\left\|\mathcal{A}_{v}^{-\alpha} \mathcal{N}(\boldsymbol{\phi}(\tau), \boldsymbol{\phi}(\tau))\right\|_{W^{s, q}}$ can be determined as follows. Because there is no coupling between $\phi_{1}$ and $\phi_{2}$ through nonlinearity in (2.11c), the nonlinear terms can be majorized with $N(\phi, \phi) \sim \nabla \phi \nabla^{3} \phi$ for $\phi$-scalar. Then using the equality $\nabla \phi \nabla^{3} \phi=\frac{1}{2} \nabla^{2}(\nabla \phi \nabla \phi)-\left(\nabla^{2} \phi\right)^{2}$ we can show that the "dominating" term of $\mathcal{N}=M^{-1} N \lesssim \nabla^{-2} N$ is $\frac{1}{2} \nabla^{2}(\nabla \phi \nabla \phi)$, because the $W^{s, q}$-norm of this term is larger than that of $\left(\nabla^{2} \phi\right)^{2}$ :

$$
\begin{aligned}
\|\mathcal{N}\|_{W^{s, q}} & \leqq\left\|\nabla^{-2}\left(\nabla \phi \nabla^{3} \phi\right)\right\| W_{W^{s, q}} \leqq \frac{1}{2}\|\nabla \phi \nabla \phi\|_{W^{s, q}}+\left\|\nabla^{-2}\left(\nabla^{2} \phi\right)^{2}\right\| W_{W^{s, q}} \\
& \leqq C_{1}\|\nabla \phi\|_{W^{s, 2 q}}^{2}+C_{2}\left\|\nabla^{2} \phi\right\|_{W^{s-2,2 q}}^{2} \leqq C_{1}\|\phi\|_{W^{s+1,2 q}}^{2}+C_{2}\|\phi\|_{W^{s, 2 q}}^{2} \\
& \leqq C\|\phi\|_{W^{s+1,2 q}}^{2} .
\end{aligned}
$$

In the above derivations we have used Lemma 3, the Schauder estimate, and the usual definitions of norms in Sobolev spaces. Hence, when estimating $\left\|\mathcal{A}_{\mathcal{v}}^{-\alpha} \mathcal{N}\right\|_{W^{s, q}}$ we will take into account only the dominating term, $\left\|\mathcal{A}_{\mathcal{v}}^{-\alpha} \mathcal{N}\right\|_{W^{s, q}} \leqq$ $C_{1}\|\nabla \phi \nabla \phi\|_{W^{s-\alpha, q}}$. Now, using the embedding of Sobolev spaces $^{12}[2]: W^{s, q / 2} \hookrightarrow$ $W^{s-\alpha, q}$, which is true for $s \geqq \alpha$ and $\alpha=2 / q$, using Lemma 3 we finally conclude that there exists a constant $C$ such that

$$
\left\|\mathcal{A}_{\mathcal{v}}^{-\alpha} \mathcal{N}(\boldsymbol{\phi}, \boldsymbol{\phi})\right\|_{W^{s, q}} \leqq C\|\boldsymbol{\phi}\|_{W^{s, q}}^{2} .
$$

Combining bounds (5.23) and (5.24), and considering the solution on its maximal time of existence (5.27), we find

$$
\|\mathcal{B}(t)\|_{W^{s, q}} \leqq C Q^{2} \delta^{2} \int_{0}^{t} \mathrm{e}^{(\lambda+\nu)(t-\tau)} \frac{1}{(t-\tau)^{\alpha}} \mathrm{e}^{2 \lambda \tau} \mathrm{d} \tau,
$$

12 The relevant part of Theorem 7.58 in ADAMs [2] states that $W^{t, p}\left(\Omega_{n} \subseteq \mathbb{R}^{n}\right) \hookrightarrow$ $W \chi, r\left(\Omega_{k} \subseteq \mathbb{R}^{k}\right)$ for $\chi=t-\frac{n}{p}+\frac{k}{r}$ where $1<p \leqq r<\infty, 1 \leqq k \leqq n, t>0$ if $\chi \geqq 0$ and $p<r$. 
which is bounded for $\alpha<1$, that is, when $q>2$, and behaves asymptotically as $\mathrm{e}^{2 \lambda t}$ since the integral in this expression is bounded for $\alpha<1$ :

$$
\int_{0}^{t} \mathrm{e}^{(\lambda+\nu)(t-\tau)} \frac{1}{(t-\tau)^{\alpha}} \mathrm{e}^{2 \lambda \tau} \mathrm{d} \tau=\mathrm{e}^{2 \lambda t} \int_{0}^{t} \frac{\mathrm{e}^{(\nu-\lambda)(t-\tau)}}{(t-\tau)^{\alpha}} \mathrm{d} \tau \leqq \mathrm{e}^{2 \lambda t} \int_{0}^{t} \frac{\mathrm{d} \tau}{(t-\tau)^{\alpha}} .
$$

Hence, the bilinear form $\mathcal{B}(t)$ grows at most like the square of the norm of $\boldsymbol{\phi}$ for as long as the latter is bounded by a constant multiple of $\mathrm{e}^{\lambda t}, \lambda>0$ :

$$
\|B(t)\|_{W^{s, q}} \leqq C\left[Q \delta \mathrm{e}^{\lambda t}\right]^{2}
$$

5.2.4. Proof of instability. Given the above properties of the linear operator $\mathcal{A}$ by Lemma 2 and the nonlinear one $\mathcal{N}$ by Lemma 4, now we turn to the proof of Theorem 3 with the help of the Duhamel formula.

Proof. In proving Lyapunov instability we will assume existence of a solution (conditional instability) and simply follow the negation of Definition 3. First, let us choose the initial condition $\phi(0)=\delta \cdot \varphi$ with the eigenfunction $\varphi \in W^{s, q}$ of $\mathcal{A}$ corresponding to the eigenvalue with the maximal real part $\lambda$ normalized as $\|\varphi\|_{W^{s, q}}=1$; since we consider the case of an instability, then $\lambda>0$. Next, let us choose a constant $Q>1$; then, by the continuity, there exists a maximal time $T(Q)$ such that

$$
\|\phi(t)\|_{W^{s, q}} \leqq Q \delta \mathrm{e}^{\lambda t} \text { for all } t \leqq T .
$$

With estimate (5.22) on $B(t)$, one can immediately demonstrate that the maximal time $T$, that is, when $\|\phi(T)\|_{W^{s, q}}=Q \delta \mathrm{e}^{\lambda t}$, is nonzero. Indeed, using the Duhamel formula (5.17):

$$
\begin{aligned}
\|\boldsymbol{\phi}(T)\|_{W^{s, q}}=Q \delta \mathrm{e}^{\lambda t} & \leqq\left\|\boldsymbol{\phi}(0) \mathrm{e}^{\mathcal{A} T}\right\|_{W^{s, q}}+\|\mathcal{B}(T)\|_{W^{s, q}} \\
& \leqq \delta \mathrm{e}^{\lambda T}+C\left[Q \delta \mathrm{e}^{\lambda T}\right]^{2}
\end{aligned}
$$

From here,

$$
\delta \mathrm{e}^{\lambda T} \geqq \frac{Q-1}{C Q^{2}},
$$

where the right-hand side will be denoted by $\chi_{*}=\delta \mathrm{e}^{\lambda t_{*}}$, for convenience. Obviously, $t_{*} \leqq T$.

The goal is to show, in accordance with the definition of Lyapunov instability, that there exists $\epsilon$ and at least one time instant $t_{1}$ when $\left\|\boldsymbol{\phi}\left(t_{1}\right)\right\|_{W^{s, q}} \geqq \epsilon$. In fact, we will demonstrate that $t_{1}=t_{*}$. Indeed, using (5.17), we find

$$
\|\boldsymbol{\phi}(t)\|_{W^{s, q}} \geqq \delta \mathrm{e}^{\lambda t}-C\left[Q \delta \mathrm{e}^{\lambda t}\right]^{2},
$$

which at $t=t_{*}$ can be rewritten as

$$
\left\|\boldsymbol{\phi}\left(t_{*}\right)\right\|_{W^{s, q}} \geqq \chi_{*}-C \chi_{*}^{2}
$$


and one can choose $Q=Q_{0}>1$ such that $\chi_{*}<1 /(2 C)$ and therefore $\left\|\phi\left(t_{*}\right)\right\|_{W^{s, q}} \geqq \chi_{*} / 2$. That is, we have proved that there exists $\epsilon=\chi_{*} / 2$ and an instant of time $t_{*}(\delta, \epsilon)$ given by $\epsilon=(\delta / 2) \mathrm{e}^{\lambda t_{*}}$, so that Lyapunov instability takes place.

In Section 7, the Lyapunov instability will be proved unconditionally upon existence of solutions in the stronger topology of $C^{k, \lambda}$, that is, strong Lyapunov instability in the sense of Definition 4, with the help of Lemma 1 and the existence results of Section 6.

5.2.5. Discussion: center-manifold theory. On the physical side these stability conclusions can be understood on the basis of center manifold reduction theory, which allows one to construct "small" solutions in the neighborhood of the critical point. The formal justification for this reduction of infinite-dimensional dynamics to its low dimensional counterpart is the center manifold theorem. For the basic discussion on the center manifold theory we refer the reader to CARR [12], CARR and Muncaster [13] and Gallay [32]. The latter two works both contain center manifold theorems for infinite-dimensional systems, but Gallay's version imposes weaker restrictions on nonlinearity. The key requirements of the center manifold theorem are (1) the linear operator restricted to the stable and unstable manifolds defines strongly continuous semigroups ( $C_{0}$ semigroups) with a spectral gap between them, and (2) the nonlinear operator is of class $\mathcal{C}^{k}, k>1$, and vanishes at the origin together with its first Fréchet derivatives. While the requirements of center manifold theory on the linear operator are usually met (in our case, it follows because of compactness of the domain $\mathbf{D}$ ), the nonlinearity is not usually of class $\mathcal{C}^{k}, k>1$, unless one has a priori a global existence theorem for a sufficiently smooth solution.

From experience (for example, [21]) it is known that this reduction usually "works" even when the theorems of the center manifold theory are not directly applicable, so that one can use center manifold theory to reduce the infinite-dimensional dynamics near a critical point to a low-dimensional manifold and then make conclusions on nonlinear stability. To sketch the derivation of such a formal theory, we transform problem (2.1) into spectral space using the basis functions (4.3) and the amplitude expansion:

$$
\phi_{i}(\mathbf{x}, t)=\sum_{m=1}^{\infty} \sum_{n=-\infty}^{+\infty} A_{m n}^{i}(t) \mathrm{e}^{i \pi n x} \sin \pi m y,
$$

which, after substitution into the full nonlinear problem and projection onto the adjoint spectral space, produces an autonomous infinite countable system of ordinary differential equations:

$$
\frac{\mathrm{d} \mathbf{A}}{\mathrm{d} t}=\Lambda \mathbf{A}+\mathbf{F}(\mathbf{A})
$$

where $\mathbf{A}$ is the infinite vector of amplitudes $A_{m n}^{i}$ defined in a suitable Banach space, $\Lambda$ is a linear operator (usually a matrix of eigenvalues) and $F_{j}(\mathbf{A})=$ 
$\sum_{n_{1}, n_{2}} \alpha_{n_{1}, n_{2}}^{(j)} A_{n_{1}} A_{n_{2}}$. As we saw from the linear stability analysis the restrictions of the linear operator $\Lambda$ to the stable (eigenvalues with $\operatorname{Re} \sigma<0$ ) and unstable (eigenvalues with $\operatorname{Re} \sigma>0$ ) subspaces generate strongly continuous semigroups, and there exists a spectral gap. According to the center manifold theorem [32] the nonlinear map $F_{j}$ should be at least $C^{1, \epsilon}$-continuous, $\epsilon>0$. This is not true in general since the global existence of a sufficiently smooth solution of (2.1) in the dissipative case and thus the convergence of these quadratic infinite sums are not known.

Once the stable and unstable manifolds are identified, system (5.30) is reduced to the equation just for the master mode $A$, which is, in our case, of Landau type and after rescaling takes the normal form

$$
\frac{\mathrm{d} A}{\mathrm{~d} t}=\sigma(\epsilon) A-|A|^{2} A+\text { h.o.t. }
$$

where $\epsilon$ is the deviation of the bifurcation parameter from its critical value $U_{c} F / 2 \beta>[2(1+\sqrt{2})]^{-1 / 2}$, such that $\sigma(\epsilon)=0$ if $\epsilon=0, \sigma(\epsilon)<0$ if $\epsilon<0$ (stable region), and $\sigma(\epsilon)>0$ if $\epsilon>0$ (unstable region).

An equation similar to (5.31) has been formally derived using perturbative analysis, for example, for an infinite strip by RomeA [75]. The Landau equation (5.31) tells us that below the marginal stability curve in the dissipative case in Fig. 5 the equilibrium solution is asymptotically stable in the Lyapunov sense, while above that curve it is unstable.

\subsection{Discussion: geometrical and physical interpretations}

In this section we provided the proof of the minimal requirements for a dissipation-induced instability to occur and all these stability results are conditional upon existence of the corresponding solutions, which will be studied in Section 6 . We have not explored other regions in Fig. 5. For example, it should be noted that while in the infinite-dimensional Hamiltonian case the spectral instability above the solid curve in Fig. 5 does not imply nonlinear instability in general, it is not required here to prove this connection in order to demonstrate the dissipationinduced instability effect. However, the reader can infer nonlinear instability in the Hamiltonian case from general theorem of FRIEDLANDER et al. [29] proved for evolution equations in Banach spaces. Their "gap" condition [29] follows from the spectral properties of the linear operator, while the properties of the nonlinear operator are discussed above.

The geometrical picture of the dissipation-induced instability can be inferred from the nonlinear Hamiltonian stability analysis in Section 5.1. Since, as we observed, the second variation of the Hamiltonian $\delta^{2} \mathrm{H}_{2}$ is definite and, since the system gets destabilized as follows from the analysis in Section 5.2, then the dissipationinduced instability corresponds to type (1.3b) and Fig. 2c of the general picture of these phenomena in finite dimensions [48].

We conclude this section with a discussion of the physical implication of the dissipation-induced instability demonstrated here. If the baroclinic instability is 
predicted by measuring the velocity difference of two layers (cf. Fig. 3) and using stability analysis of the Hamiltonian formulation, then the error of this forecast would be around $10 \%$, as follows from a comparison of the critical bifurcation parameters, $1 / 2$ and $[2(1+\sqrt{2})]^{-1 / 2}$ (cf. Figs. 4,5 ), for conservative and dissipative formulations respectively.

\section{Existence of classical solutions}

In order to make the results obtained in Section 5 on nonlinear stability and instability unconditional, we still need to demonstrate that the solution exists, which would complete the proof of the main Theorem 1. Formally speaking, local existence suffices, but extending existence to any finite time interval turns out to be relatively straightforward with some additional assumptions on the initial data. We work out the existence proof for classical solutions, as motivated by their direct physical significance; this will automatically make the nonlinear stability estimates in the Hamiltonian case applicable, cf. Section 5.1, though such strong smoothness requirements may be a bit conservative. The existence study is performed here using standard PDE analysis and a priori estimates technique, that is the philosophy going back to BERNSTEIN [8]. In Section 6.1 we prove the local existence theorem in the Hamiltonian case and in Section 6.2 we extend it to the dissipative case. The proof is basically patterned after KATO's work [44] on the Euler equation. That is, we also appeal to the fixed point theorem (second Schauder theorem), and construct the solution iterates directly from the fluid particle paths instead of using the conventional Galerkin method. This approach also allows us to highlight some solution properties. Section 6.3 is devoted to the globalization of the existence result, which is achieved by obtaining a global in time estimate and successive application of the local existence theorem. As mentioned in the introduction, our goal here is not to advance the existence theory for quasi-geostrophic equations in a significant way, but rather to make the stability results unconditional. Hence, in Sections 6.1 and 6.3 we largely follow the early existence studies of the conservative quasi-geostrophic equations on doubly periodic domains in the case of continuous stratification by DUTTON [24] (who showed the local existence of weak solutions), BENNETT and KLOEDEN [7] (who obtained the local existence of classical solutions) and by BourgeoIs and BEALE [10] (who proved the global existence of weak solutions). However, here we extend these results to the channeltype boundary conditions, cf. Section 2.1, and the dissipative quasi-geostrophic equation (2.1), and prove the existence results for classical solutions.

For the purpose of the proof, the original system (2.1) in the conservative case, $r=0$, is decomposed into a boundary value problem - the following system of elliptic equations:

$$
\begin{aligned}
& \nabla^{2} \widetilde{\psi}_{1}-F\left(\widetilde{\psi}_{1}-\widetilde{\psi}_{2}\right)=\zeta_{1}, \\
& \nabla^{2} \widetilde{\psi}_{2}+F\left(\widetilde{\psi}_{1}-\psi_{2}\right)=\zeta_{2},
\end{aligned}
$$




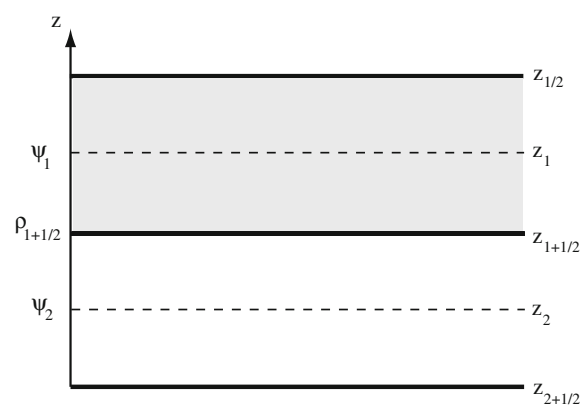

Fig. 7. On the discretized elliptic operator

or $M \widetilde{\psi}=\zeta$ with $M$ defined by (2.11a), that are periodic in $x$ and have boundary conditions $(2.2-2.3)$, and into the initial value problem for vorticity $\widetilde{\zeta}_{i}$ :

$$
\begin{aligned}
\left(\partial_{t}+\widetilde{u}_{i} \partial_{x}+\widetilde{v}_{i} \partial_{y}\right) \widetilde{\zeta}_{i} & =-\beta \widetilde{v}_{i}, i=1,2, \\
\left.\widetilde{\zeta}_{i}\right|_{t=0} & =\widetilde{\zeta}_{i 0} .
\end{aligned}
$$

Note the placement of tildas: first one solves the elliptic problem (6.1) and gets $\widetilde{\psi}$ for given $\zeta$ and then from (6.2) one determines $\widetilde{\zeta}$. This defines a map

$$
\mathcal{T}: \zeta \mapsto \widetilde{\zeta}
$$

which, as we will show, satisfies the hypothesis of the second Schauder fixed point theorem and thus proves the existence of a solution in the function space to be specified later, in Section 6.1.

Before proceeding, we need to demonstrate the uniqueness of the solution for (6.1). For this it is enough to explore the operator $\mathcal{G}$, which is an algebraic part of the operator on left-hand side of (6.1),

$$
\mathcal{G}=F\left(\begin{array}{cc}
-1 & 1 \\
1 & -1
\end{array}\right) \Rightarrow(\mathcal{G} \boldsymbol{\xi}, \boldsymbol{\xi})=-F\left(\xi_{1}-\xi_{2}\right)^{2},
$$

which is obviously negative semi-definite, and thus analogous to a discrete elliptic operator. If $F>0$, then there are two eigenvalues, $(-2 F, 0)$, which are simple. Therefore, one can conclude that the operator $\nabla^{2}+\mathcal{G}$ comes from discretizing the continuous stratification operator $\mathcal{G}_{c}(z)=\partial_{z}\left(\rho(z) \partial_{z} \psi\right)$, as shown in Fig. 7. The latter was probably not obvious from the derivation in Appendix A where two superimposed layers of different density were considered to interact through interfacial conditions. Indeed, if the coordinates and stream-functions at the center of each of two layers are $\left(z_{1}, \psi_{1}\right)$ (upper) and $\left(z_{2}, \psi_{2}\right)$ (lower) with the coordinates $z_{1 / 2}, z_{2+1 / 2}$ and $z_{1+1 / 2}$ at the top of the upper, bottom of the lower and in between the layers respectively, then discretizing the continuous operator gives

$$
\begin{aligned}
\mathcal{G}_{d}= & \frac{2}{h_{1}+h_{2}}\left[\rho\left(z+\frac{h_{1}}{2}\right) \frac{\psi\left(z+h_{1}\right)-\psi(z)}{h_{1}}\right. \\
& \left.-\rho\left(z-\frac{h_{2}}{2}\right) \frac{\psi(z)-\psi\left(z-h_{2}\right)}{h_{2}}\right],
\end{aligned}
$$


where $h_{1}$ and $h_{2}$ stand for the distance between the points of one-sided derivatives. Next we use the fact that at the top and at the bottom:

$$
\left.\frac{\partial \psi}{\partial z}\right|_{z_{1 / 2}} \simeq \frac{\psi\left(z_{1 / 2}\right)-\psi\left(z_{1}\right)}{\Delta z / 2}=0,\left.\quad \frac{\partial \psi}{\partial z}\right|_{z_{2+1 / 2}}=\frac{\psi\left(z_{2}+1 / 2\right)-\psi\left(z_{2}\right)}{\Delta z / 2}=0,
$$

where $\Delta z=z_{2}-z_{1}$ is the thickness of identical layers and thus the distance between their centers. Applying $\mathcal{G}_{d}(z)$ to the centers of each layer, $z_{1}$ and $z_{2}$, we find

$$
\begin{array}{ll}
\mathcal{G}_{d}\left(z_{1}\right)=\frac{4 \rho\left(z_{1+1 / 2}\right)}{3 \Delta z} \frac{\psi\left(z_{2}\right)-\psi\left(z_{1}\right)}{\Delta z}, & h_{1}=\Delta z, h_{2}=\Delta z / 2, \\
\mathcal{G}_{d}\left(z_{2}\right)=-\frac{4 \rho\left(z_{1+1 / 2}\right)}{3 \Delta z} \frac{\psi\left(z_{2}\right)-\psi\left(z_{1}\right)}{\Delta z}, & h_{1}=\Delta z / 2, h_{2}=\Delta z,
\end{array}
$$

and thus arrive at system (6.1) with $F=4 \rho\left(z_{1+1 / 2}\right) / 3 \Delta z^{2}$.

The ellipticity of (6.1) implies that vorticity is related to stream-function by a three-dimensional elliptic operator and thus the properties of the quasi-geostrophic model are very much like those of the three-dimensional Euler equation. It is because of the quite simplified vertical structure (only two layers) and the absence of pressure that one can get global existence, as opposed to the three-dimensional Euler equation where this issue is still unresolved. Finally, we note that the duality of interpretation of the zero order elliptic operator $\mathcal{G}$, as coming from the potential energy (shown in the original derivation, Appendix A) and as following from the discretization of elliptic operator (shown above), that is, kinetic energy, has an interesting consequence - the Langrangian and the Hamiltonian are identical in the case of zero $\beta$-effect (similar to the Euler equation for an incompressible fluid).

Finally, before proceeding with the proofs we need to provide the functional set-up: the principal flow domain, $\mathbf{D}$, is a convex subset of $\mathbb{R}^{2}$. Using the structure of the solution, that is, anti-symmetry in $y$ and periodicity of $\phi_{i}$, this domain can be extended to square $\widehat{\mathbf{D}}=\{-1 \leqq x, y \leqq 1\}$. In view of double-periodicity of the solution, its lateral boundaries can be considered as virtual boundaries, because the boundary points are interior points of $\widehat{\mathbf{D}}$ with the appropriate shift. Therefore we can identify the flow domain $\widehat{\mathbf{D}}$ with torus $\mathbf{T}^{2}$ with the appropriate equivalence relation. This duality of interpretation of the domain $\widehat{\mathbf{D}}$ will facilitate the use of elliptic estimates necessary for the existence analysis, once it is considered as torus $\mathbf{T}^{2}$, and of imbedding theorems, once it is considered as a convex domain in $\mathbb{R}^{2}$. With this understanding, we will uniformly use the notation $\mathbf{T}^{2}$, even though the torus is not a convex set. In choosing the spaces we follow the observation of SCHAUDER [79] that appropriate estimates in the quasi-linear case can be Hölder $\mathcal{C}^{2, \lambda}$ estimates, where $\mathcal{C}^{s, \lambda}$ is defined Section 2.3. For the definitions of other spaces used throughout this section refer to Section 2.3.

In the following local existence analysis, Sections 6.1 and 6.2, we make all estimates for $s=0$. All the results can be extended onto general case of $\mathcal{C}^{s, \lambda}$ in a straightforward manner: larger $s$ corresponds to smoother initial data and thus to smoother solutions. This fact will be used in the proof of a global solution in Section 6.3. 


\subsection{Local existence in the Hamiltonian case}

First consider the elliptic problem (6.1) and treat time as a parameter. As mentioned above, we provide all estimates only for $s=0$ in $\mathcal{C}^{s, \lambda}$ which is enough for establishing the existence of the classical solution. If $\zeta \in \mathcal{C}^{0, \lambda}$, then there exists a unique solution $\widetilde{\psi} \in \mathcal{C}^{2, \lambda}$ of the elliptic problem (6.1) satisfying the condition of periodicity in $x$ and boundary conditions (2.2-2.3), such that the following global Schauder estimate is valid

$$
\|\widetilde{\boldsymbol{\psi}}\|_{\mathcal{C}^{2, \lambda}} \leqq K_{1}\|\zeta\|_{\mathcal{C}^{0, \lambda}}
$$

where the constant $K_{1}$ is independent of $\zeta$ and $\widetilde{\psi}$, but depends on $\lambda$ and $\mathbf{T}^{2}$. For an introductory discussion on Schauder estimates we refer the reader to CoURANT and Hilbert [20], and for the derivation to Krylov [51], LADYZHENSKAYA and UraL' tSEVA [52], and Miranda [63].

Since $\mathbf{T}^{2}$ is a compact set that is the quotient of a convex set, the space $\mathcal{C}^{1}$ is compactly imbedded in $\mathcal{C}^{0, \lambda}$ [2], so that

$$
\|\zeta\|_{\mathcal{C}^{0, \lambda}} \leqq\|\zeta\|_{\mathcal{C}^{1}}\left(\operatorname{diam} \mathbf{T}^{2}\right)^{1-\lambda}
$$

and the Schauder estimate (6.5) can be replaced with

$$
\|\widetilde{\boldsymbol{\psi}}\|_{\mathcal{C}^{2, \lambda}} \leqq K_{2}\|\zeta\|_{\mathcal{C}^{1}}, \quad K_{2}=K_{1}\left(\operatorname{diam} \mathbf{T}^{2}\right)^{1-\lambda}
$$

Next, in order to resolve the initial value problem (6.2) with initial condition $\zeta \in \mathcal{C}^{1}$ (since we need $\zeta$ to be differentiable at least once), we need to introduce a convex subspace $\mathcal{M}(T)$ of $\widehat{\mathcal{C}}_{0}^{1}$ (note that we switch to time-dependent spaces) defined through

$$
\mathcal{M}(T)=\left\{\zeta \in \widehat{\mathcal{C}}_{0}^{1} \text { with }\|\zeta\|_{\widehat{\mathcal{C}}_{0}^{1}} \leqq L_{1}(1+\epsilon)\right\},
$$

where $\epsilon>0$ is an arbitrary, but fixed number, and $L_{1}$ is determined in the course of making estimates on vorticity (see Appendix $C$ ). We will also need a subset $\mathcal{K}(T)$ of $\mathcal{M}(T)$ defined by

$$
\mathcal{K}(T)=\left\{\zeta \in \mathcal{M}(T), \partial_{t} \zeta \in \widehat{\mathcal{C}}_{0}^{0},\left\|\partial_{t} \zeta\right\|_{\widehat{\mathcal{C}}_{0}^{0}} \leqq K_{3} L_{1}(1+\epsilon) \mathrm{e}^{2 K_{3}(1+\epsilon) T}\right\} .
$$

Finally, we shall demonstrate that the time of existence $T^{*}$ of the solution is inversely proportional to the norm of initial vorticity:

$$
T^{*}=L_{1}^{-1} \ln (1+\epsilon)\left[K_{2}(1+\epsilon)\right]^{-1},
$$

and that the mapping $\mathcal{T}$ (6.3), the fixed point of which is a solution of (2.1), is well-defined from $\mathcal{M}\left(T^{*}\right)$ into its subset $\mathcal{K}\left(T^{*}\right)$. This all together will give the local existence in the Hamiltonian case: 
Theorem 4. Suppose that the initial vorticity $\zeta^{I} \in \mathcal{C}^{1}$ and $T^{*}$ is defined by (6.10). Then there exists a unique solution to the quasi-geostrophic equation (2.1), when $r=0$, for the time interval $\left[0, T^{*}\right]$ in the space

$$
X=\left\{\zeta \in \widehat{\mathcal{C}}^{1}, \text { and } \psi \in \widehat{\mathcal{C}}_{0}^{2, \lambda}\right\},
$$

where the norm on $X$ is a Hölder norm, defined as in Section 2.3.

This result will be proved in the following subsections.

6.1.1. Elliptic problem. For any $\zeta \in \mathcal{M}(T)$ and each $t \in[0, T]$, the periodic boundary-value problem (6.1) has a unique solution $\widetilde{\psi} \in \mathcal{C}^{2, \lambda}$ which satisfies the Schauder estimate (6.7), where $K_{2}$ is independent of $t$ and $T$. Therefore, and in view of the linearity of this boundary-value problem, we have

$$
\left\|\widetilde{\boldsymbol{\psi}}\left(t^{\prime}\right)-\widetilde{\boldsymbol{\psi}}\left(t^{\prime \prime}\right)\right\|_{\mathcal{C}^{2, \lambda}} \leqq K_{2}\left\|\zeta\left(t^{\prime}\right)-\zeta\left(t^{\prime \prime}\right)\right\|_{\mathcal{C}^{1}},
$$

for any $t^{\prime}, t^{\prime \prime} \in[0, T]$. Thus the stream-function $\widetilde{\psi}$ is uniformly continuous on $[0, T]$ in the $\mathcal{C}^{2, \lambda}$ norm as long as $\zeta \in \mathcal{M}(T)$. Taking suprema over $0 \leqq t \leqq T$ in the Schauder estimate (6.7) yields

$$
\|\widetilde{\boldsymbol{\psi}}\|_{\widehat{\mathcal{C}}_{0}^{2, \lambda}} \leqq K_{2}\|\zeta\|_{\widehat{\mathcal{C}}_{0}^{1}}
$$

and, recalling definition (6.8),

$$
\|\widetilde{\boldsymbol{\psi}}\|_{\widehat{\mathcal{C}}_{0}^{2, \lambda}} \leqq K_{2} L_{1}(1+\epsilon)=K_{3}(1+\epsilon) \text {, that is, } \widetilde{\psi} \in \widehat{\mathcal{C}}_{0}^{2, \lambda} \text {. }
$$

Once the stream-function is defined, one can calculate the velocity field which in each layer is obviously defined (omitting indices) as

$$
\mathbf{v}=(u, v)=\left(-\partial_{y} \psi, \partial_{x} \psi\right) \in \widehat{\mathcal{C}}_{0}^{1, \lambda},
$$

with bound

$$
\|\mathbf{v}\|_{\widehat{\mathcal{C}}_{0}^{1, \lambda}} \leqq K_{3}(1+\epsilon) .
$$

These velocity components allow one to formulate a two-dimensional system of ordinary-differential equations which define the quasi-geostrophic fluid particle paths in each layer

$$
\begin{aligned}
\mathrm{d}_{\tau} \xi & =u(\xi, \eta, \tau), \\
\mathrm{d}_{\tau} \eta & =v(\xi, \eta, \tau),
\end{aligned}
$$

so that at the final time $\tau=t: \xi=x, \eta=y$. The transition to Lagrangian variables $\xi=(\xi, \eta)$ will allow us to construct the solution of initial-value problem (6.2) explicitly [4]. System (6.17) is the classical example of a system of ODEs on a torus (cf. HARTMAN [38]), which has a unique solution for all $(x, y) \in \mathbf{T}^{2}$ and $0 \leqq \tau \leqq t \leqq T^{*}$

$$
\xi=\xi(\tau ; x, y, t), \eta=\eta(\tau ; x, y, t) \Leftrightarrow x=x(t ; \xi, \eta, \tau), y=y(t ; \xi, \eta, \tau),
$$


which is differentiable with respect to all parameters (cf. HARTMAN [38], Theorem V3.1): $\xi \in C^{1}\left(\mathbf{T}^{2} \times[0, T] \times[0, T]\right)$. Restricting this to $\tau=0$, we get $\xi \in \widehat{\mathcal{C}}^{1}$. This however, as will follow from the bounds below (see also EBIN and MARSDEN [26]), can be strengthened to

$$
(\xi, \eta) \in \widehat{\mathcal{C}}_{0}^{1, \lambda},\left(\partial_{t} \xi, \partial_{t} \eta\right) \in \widehat{\mathcal{C}}_{0}^{0, \lambda}
$$

that is smoother than $\widehat{\mathcal{C}}_{0}^{1}$ and $\widehat{\mathcal{C}}_{0}^{0}$ respectively, as follows from estimates $(6.20 \mathrm{~b}-$ 6.20c). For $\tau=0$ the solution satisfies the following bounds derived in Appendix B,

$$
\begin{gathered}
\|\xi\|_{\widehat{\mathcal{C}}_{0}^{0}} \leqq K_{3}(1+\epsilon) T+1, \\
\|\nabla \xi\|_{\widehat{\mathcal{C}}_{0}^{0}} \leqq \mathrm{e}^{2 K_{3}(1+\epsilon) T}, \\
{[\nabla \xi]_{\widehat{\mathcal{C}}_{0}^{0, \lambda}} \leqq \mathrm{e}^{(3+2 \lambda) K_{3}(1+\epsilon) T},} \\
\left\|\partial_{t} \xi\right\|_{\widehat{\mathcal{C}}_{0}^{0}} \leqq K_{3}(1+\epsilon) \mathrm{e}^{2 K_{3}(1+\epsilon) T}, \\
{\left[\partial_{t} \xi\right]_{\widehat{\mathcal{C}}_{0}^{0, \lambda}} \leqq 2 K_{3}(1+\epsilon) \mathrm{e}^{(3+2 \lambda) K_{3}(1+\epsilon) T},}
\end{gathered}
$$

where, obviously, the semi-norm $[\cdot]_{\widehat{\mathcal{C}}_{0}^{0, \lambda}}=\|\cdot\|_{\widehat{\mathcal{C}}_{0}^{0, \lambda}}$. From the properties of the solution for (6.17) it follows that the change of the final time condition $(x, y, t) \rightarrow$ $\left(x^{\prime}, y^{\prime}, t^{\prime}\right)$ does not change $\xi$ and $\eta$, that is,

$\mathrm{d}_{t} \xi=\xi_{t}+\frac{\mathrm{d} x}{\mathrm{~d} t} \xi_{x}+\frac{\mathrm{d} y}{\mathrm{~d} t} \xi_{y}=\left(\partial_{t}+u(x, y, t) \partial_{x}+v(x, y, t) \partial_{y}\right) \xi(\tau ; x, y, t)=0$, and same for $\eta$. In particular, this remains valid for $\tau=0$ :

$$
\begin{aligned}
& \left.\mathrm{d}_{t} \xi\right|_{\tau=0}=0, \\
& \left.\mathrm{~d}_{t} \eta\right|_{\tau=0}=0 .
\end{aligned}
$$

This property will be used in constructing a solution for the initial value problem (6.2).

6.1.2. Initial value problem. The initial value problem (6.2) is solved using fluid particle paths at $\tau=0$ :

$$
\widetilde{\zeta}(x, y, t)=\zeta^{I}(\xi(0 ; x, y, t), \eta(0 ; x, y, t))+\beta[\eta(0 ; x, y, t)-y],
$$

which is a unique solution of (6.2) in view of the assumption $\zeta^{I} \in \mathcal{C}^{1}$ and Equation (6.21); (6.22) at $t=0$ is obviously $\widetilde{\zeta}(x, y, 0)=\zeta^{I}(x, y)$. The function $\widetilde{\zeta}(x, y, t)$ is the first iterate of the solution $\zeta(x, y, t)$. In Appendix $\mathrm{C}$ it is shown that $\widetilde{\zeta}(x, y, t)$ satisfies the bounds

$$
\begin{aligned}
\|\widetilde{\zeta}\|_{\widehat{\mathcal{C}}_{0}^{1}} & \leqq L_{1} \mathrm{e}^{2 K_{3}(1+\epsilon) T}, \\
\left\|\partial_{t} \widetilde{\zeta}\right\|_{\widehat{\mathcal{C}}_{0}^{0}} & \leqq K_{3} L_{1}(1+\epsilon) \mathrm{e}^{2 K_{3}(1+\epsilon) T},
\end{aligned}
$$

where $T$ needs to be defined to be consistent with (6.8). From estimate (6.23b) we are led to the definition of $\mathcal{K}(T)$ introduced in (6.9). From (6.23a) and (6.8) we get the definition for the length of the time interval $T^{*}(6.10)$. 
6.1.3. Proof of Theorem 4. As follows from bounds (6.23), if $\zeta^{I} \in \mathcal{M}\left(T^{*}\right)$ then $\widetilde{\zeta} \in \mathcal{K}\left(T^{*}\right)$, which is a subset of $\mathcal{M}\left(T^{*}\right)$ by definition. Therefore, the mapping (6.3) is well-defined from $\mathcal{M}\left(T^{*}\right)$ into its subset $\mathcal{K}\left(T^{*}\right)$. From the definition of $\mathcal{K}(T)$ it is a nonempty, closed and bounded subset of $\widehat{\mathcal{C}}^{1}$ and $\widehat{\mathcal{C}}_{0}^{1}$. Since both space $\mathbf{T}^{2}$ and the time domains $\left[0, T^{*}\right]$ are bounded and convex sets, it follows, cf. ADAms [2], that the intersection of $\widehat{\mathcal{C}}^{1}$ and $\widehat{\mathcal{C}}_{0}^{1}$ is compactly imbedded in $\widehat{\mathcal{C}}_{0}^{0, v}$ for any $0<v<\lambda$, that is, $\mathcal{K}\left(T^{*}\right)$ is a nonempty compact subset of $\widehat{\mathcal{C}}_{0}^{0, v}$.

Now we need to make sure that the mapping $(6.3), \mathcal{T}: \zeta \mapsto \widetilde{\zeta}$, is continuous in the normed space $\widehat{\mathcal{C}}_{0}^{0, v}$ with $v$ fixed. Let

$$
\begin{aligned}
& \zeta_{n} \rightarrow \zeta^{*}: \text { convergence, } \\
& \widetilde{\zeta}_{n}=\mathcal{T}\left(\zeta_{n}\right): \text { iterations, } \\
& \widetilde{\zeta}^{*}=\mathcal{T}\left(\zeta^{*}\right): \text { limit. }
\end{aligned}
$$

Since a $\widehat{\mathcal{C}}^{0, v}$ norm can be estimated by the product of the $\widehat{\mathcal{C}}^{0}$ and $\widehat{\mathcal{C}}^{0, \lambda}$ norms (cf. ADAMs [2]), the convergence in a $\widehat{\mathcal{C}}^{0, v}$ norm of a sequence belonging to a set which is bounded in $\widehat{\mathcal{C}}^{0, \lambda}$ norm can be proved by demonstrating the convergence in the $\widehat{\mathcal{C}}^{0}$ norm. In our case, the sequence $(6.24 \mathrm{~b})$ and the proposed limit $(6.24 \mathrm{c})$ belong to $\mathcal{K}\left(T^{*}\right)$ which is bounded in the norm of $\widehat{\mathcal{C}}_{0}^{1}$. Thus the convergence

$$
\widetilde{\zeta}_{n} \rightarrow \widetilde{\zeta}^{*}
$$

in the norm $\widehat{\mathcal{C}}_{0}^{0, v}$ will follow from the convergence in the norm of $\widehat{\mathcal{C}}^{0}$. Let $\psi_{n}$ and $\psi^{*}$ be the stream-functions corresponding to $\zeta_{n}$ and $\zeta^{*}$. Using linearity of (6.1) and the Schauder estimate (6.5):

$$
\left\|\psi_{n}-\psi^{*}\right\|_{\widehat{\mathcal{C}}_{0}^{2, v}} \leqq K_{1}(v)\left\|\zeta_{n}-\zeta^{*}\right\|_{\widehat{\mathcal{C}}_{0}^{0, v}}
$$

we get

$$
\psi_{n} \rightarrow \psi^{*} \text { in } \widehat{\mathcal{C}}_{0}^{2, v} \text { and } \mathbf{v}_{n} \rightarrow \mathbf{v}^{*} \text { in } \widehat{\mathcal{C}}_{0}^{1, v} .
$$

Therefore (see HARTMAN [38]), in view of existence and uniqueness for (6.17):

$$
\xi_{n} \rightarrow \xi^{*} \text { in } \widehat{\mathcal{C}}_{0}^{1} \text {. }
$$

Since

$$
\left\|\zeta_{n}-\zeta^{*}\right\|_{\widehat{\mathcal{C}}_{0}^{0}} \leqq\left\|\nabla \zeta^{I}\right\|_{\mathcal{C}^{0}}\left\|\xi_{n}-\xi^{*}\right\|_{\widehat{\mathcal{C}}_{0}^{0}}
$$

the convergence (6.25) follows from (6.28) and the preceding bound. Consequently the mapping $\mathcal{T}$ is continuous in the norm $\widehat{\mathcal{C}}_{0}^{0, v}$.

Based on the second fixed point theorem of Schauder (cf. SMART [81]), which states that if $\mathcal{M}\left(T^{*}\right)$ is a nonempty convex subset of a normed space $\widehat{\mathcal{C}}_{0}^{0, v}$ and if $\mathcal{T}$ is a continuous mapping of $\mathcal{M}\left(T^{*}\right)$ into a compact subset $\mathcal{K}\left(T^{*}\right)$, then $\mathcal{T}$ has a fixed point:

$$
\mathcal{T}\left(\zeta^{*}\right)=\zeta^{*}
$$

we prove that the periodic quasi-geostrophic problem for $0 \leqq t \leqq T^{*}$ has a solution (and the corresponding stream-function $\psi^{*}$ ), which satisfies the conclusions of Theorem 4. 


\subsection{Local existence in the dissipative case}

To prove local existence in the dissipative case we need either to show that the estimates on vorticity (6.23) remain valid for $r>0$, which one would expect to hold for small enough dissipation, or to get modified estimates and change the spaces (6.8-6.9). Since small dissipation suffices for our purposes, we will try to show the validity of (6.23).

The previous iteration scheme consisting of the elliptic problem (6.1) and the initial value problem (6.2) remains the same with the exception that (6.2) is modified to

$$
\begin{aligned}
\left(\partial_{t}+\widetilde{u}_{i} \partial_{x}+\widetilde{v}_{i} \partial_{y}\right) \widetilde{\zeta}_{i}+r \widetilde{\zeta}_{i} & =-\beta \widetilde{v}_{i}+r(-1)^{i} F\left(\widetilde{\psi}_{1}-\widetilde{\psi}_{2}\right), \\
\left.\widetilde{\zeta}_{i}\right|_{t=0} & =\widetilde{\zeta}_{i 0},
\end{aligned}
$$

where $i=1,2$, which can be transformed with $\widetilde{\zeta}_{i}=\mathrm{e}^{-r t} \widehat{\zeta}_{i}$ to

$$
\left(\partial_{t}+\widetilde{u}_{i} \partial_{x}+\widetilde{v}_{i} \partial_{y}\right) \widehat{\zeta}_{i}=\mathrm{e}^{r t}\left[-\beta \widetilde{v}_{i}+r(-1)^{i} F\left(\widetilde{\psi}_{1}-\widetilde{\psi}_{2}\right)\right] .
$$

The solution for this problem is given by

$$
\begin{aligned}
\widehat{\zeta}(x, y, t)= & \widehat{\zeta}^{I}(\xi(0 ; x, y, t), \eta(0 ; x, y, t))+\beta\left[\eta(0 ; x, y, t)-\mathrm{e}^{r t} y\right] \\
& +r \int_{0}^{t}\left\{\beta \eta(\tau ; x, y, t)+F(-1)^{i}\left[\widetilde{\psi}_{1}-\widetilde{\psi}_{2}\right](\xi(\tau ; x, y, t), \tau)\right\} \mathrm{e}^{r \tau} \mathrm{d} \tau .
\end{aligned}
$$

As shown in Appendix D, estimates (6.23) with dissipation become

$$
\begin{aligned}
\|\widetilde{\zeta}\|_{\widehat{\mathcal{C}}_{0}^{1}} & \leqq \widetilde{L}_{1} \mathrm{e}^{2 K_{3}(1+\epsilon) T}+r T \mathrm{e}^{r T} A, \\
\left\|\partial_{t} \widetilde{\zeta}\right\|_{\widehat{\mathcal{C}}_{0}^{0}} & \leqq K_{3} \widetilde{L}_{1}(1+\epsilon) \mathrm{e}^{2 K_{3}(1+\epsilon) T}+r T \mathrm{e}^{r T} B,
\end{aligned}
$$

where the first terms are the original estimates (6.23) in the conservative case with $\widetilde{L}_{1}$ being explained below and where

$$
\begin{aligned}
& A=2\left\{\beta+F K_{3}(1+\epsilon)\right\}\left(1+\mathrm{e}^{2 K_{3}(1+\epsilon) T}\right), \\
& B=\frac{\mathrm{e}^{-r T}}{T}\|\widehat{\zeta}\|_{\widehat{\mathcal{C}}_{0}^{0}}+\frac{2}{T}\left\{\beta+F K_{3}(1+\epsilon)\right\}\left(1+K_{3}(1+\epsilon) T \mathrm{e}^{2 K_{3}(1+\epsilon) T}\right) .
\end{aligned}
$$

The vorticity norm $\|\widehat{\zeta}\|_{\widehat{\mathcal{C}}_{0}^{0}}$ in the above expression can be estimated from (6.33),

$$
\|\widehat{\zeta}\|_{\widehat{\mathcal{C}}_{0}^{0}} \leqq\left\|\zeta^{I}\right\|_{\mathcal{C}^{1}}+2 \beta+2 r T \mathrm{e}^{r T}\left\{\beta+F K_{3}(1+\epsilon)\right\} .
$$

Now we are prepared to demonstrate that the original estimates (6.23) remain essentially the same after the addition of small dissipation. To show this we use the fact that estimates (6.23) are too conservative; let us look at Equations (C.1-C.2), and their combination (C.3):

$$
\begin{aligned}
\|\widetilde{\zeta}\|_{\widehat{\mathcal{C}}_{0}^{1}} & \leqq\|\widetilde{\zeta}\|_{\widehat{\mathcal{C}}_{0}^{0}}+\left\|\nabla_{\mathbf{x}} \widetilde{\zeta}\right\|_{\widehat{\mathcal{C}}_{0}^{0}}=\left\|\zeta^{I}\right\|_{\mathcal{C}^{1}}+3 \beta+\left[\left\|\zeta^{I}\right\|_{\mathcal{C}^{1}}+\beta\right] \mathrm{e}^{2 K_{3}(1+\epsilon) T} \\
& =\left[2\left\|\zeta^{I}\right\|_{\mathcal{C}^{1}}+4 \beta\right] \mathrm{e}^{2 K_{3}(1+\epsilon) T}+\left[\left\|\zeta^{I}\right\|_{\mathcal{C}^{1}}+3 \beta\right]\left(1-\mathrm{e}^{2 K_{3}(1+\epsilon) T}\right),
\end{aligned}
$$


that is $\|\widetilde{\zeta}\|_{\widehat{\mathcal{C}}_{0}^{1}} \leqq \widetilde{L}_{1} \mathrm{e}^{2 K_{3}(1+\epsilon) T}$ with the obvious definition of $\widetilde{L}_{1}<L_{1}$, since $1-$ $\mathrm{e}^{2 K_{3}(1+\epsilon) T}<0$ and $L_{1}=2\left\|\zeta^{I}\right\|_{\mathcal{C}^{1}}+4 \beta$ as in (6.23a). Now, choosing dissipation $r$ such that

$$
\max r T \mathrm{e}^{r T}(A, B) \leqq\left[\left\|\zeta^{I}\right\|_{\mathcal{C}^{1}}+3 \beta\right]\left(\mathrm{e}^{2 K_{3}(1+\epsilon) T}-1\right)
$$

we get exactly the same estimates (6.23) with the original definition of $L_{1}$, and thus the local existence proof remains valid in the dissipative case:

Theorem 5. Suppose that the initial vorticity $\zeta^{I} \in \mathcal{C}^{1}$ and $T^{*}$ is defined by (6.10). Then there exists a unique classical solution in the space $X$, defined by (6.11), to the quasi-geostrophic equation (2.1), when $r>0$, for the time interval $\left[0, T^{*}\right]$ with

$$
\zeta \in \widehat{\mathcal{C}}^{1}, \text { and } \psi \in \widehat{\mathcal{C}}_{0}^{2, \lambda} \text {. }
$$

While now we have the proof of local existence in the dissipative case, influence of dissipation on the dynamics of (2.1) is not trivial. As mentioned in Appendix A, the Eckman layer dissipation acts like a sink of potential vorticity [70], but this is true only in the $L_{2}$-sense and only for special structure of the solution and boundary conditions as we learned from the energy balance Equation (3.3). What dissipation does pointwise is not really transparent as opposed to the trivialized version of (2.1), namely

$$
\begin{aligned}
& \partial_{t} \omega+u \partial_{x} \omega=-r \omega, \\
& t=0: \omega=f(x) .
\end{aligned}
$$

Consider, for a moment, the linear version as the iterative approximation (6.2). Then the general solution of (6.39) obtained by the method of characteristics is $\omega(t, x)=f(x-u t) \mathrm{e}^{-r t}$. Therefore the problem (6.39) admits the transformation:

$$
\omega(t, x)=\widetilde{\omega}(t, x) \mathrm{e}^{-r t} \Rightarrow \partial_{t} \widetilde{\omega}+u \partial_{x} \widetilde{\omega}=0 .
$$

Thus the problem of demonstrating existence in that trivialized situation (6.39) reduces to the conservative case and the solutions decay in time according to the above transformation. However, the two-layer structure (2.1) with the $\beta$-plane effect has an interaction which destroys this simple pointwise sinkage of vorticity.

\subsection{Global existence in the Hamiltonian case}

The basic idea to get global existence of classical solutions is first to obtain a global $L^{2}$ bound on weak solutions in a Sobolev space $\mathcal{H}^{s}$ with sufficiently high enough index $s,{ }^{13}$ so that one can exploit the Sobolev inequality in the twodimensional case $|f|_{\mathcal{C}^{k}} \leqq C\|f\|_{\mathcal{H}^{k+s}}$ to get a pointwise bound on a lower derivative, cf. Adams [2]. The latter guarantees the existence of the classical solution if $s \geqq 1$. Thus, for global existence we need to add to the assumptions in the local existence Theorem 4 an assumption that the initial data lies in $\mathcal{H}^{s}, s \geqq 3$.

${ }^{13}$ Note that this index is different from the one used in spaces $\mathcal{C}^{s, \lambda}, \widehat{\mathcal{C}}_{0}^{s, \lambda}$ or $\widehat{\mathcal{C}}^{s, \lambda}$ previously. 
Lemma 5. The solution of (2.1), when $r=0$, for initial data in $\mathcal{H}^{s}, s \geqq 3$, remains bounded in $\mathcal{H}^{s}$ on any finite time interval $0 \leqq t \leqq T<\infty$.

Proof. We will prove this by showing that there exists a continuous function $Z(t)$ such that

$$
\|\zeta\|_{\mathcal{H}^{s}} \leqq Z(t), \quad 0 \leqq t \leqq T<\infty .
$$

Elliptic theory in Sobolev spaces [34] gives

$$
\|\boldsymbol{v}\|_{\mathcal{H}^{s+1}} \leqq C_{1}\|\zeta\|_{\mathcal{H}^{s}} .
$$

Applying the operator $D^{\alpha},|\alpha| \leqq s$ to $(2.1)$, that is, $\left(\partial_{t}+\mathbf{v} \nabla\right) \zeta=-\beta v$, where indexes are omitted, we get

$$
\left(\partial_{t}+\mathbf{v} \nabla\right) \zeta_{\alpha}=-F_{\alpha},
$$

where $\zeta_{\alpha}=D^{\alpha} \zeta, F_{\alpha}=\beta v_{\alpha}+D^{\alpha} \nabla(\mathbf{v} \zeta)-\mathbf{v} D^{\alpha} \nabla \zeta$. Now we can get a bound on $F_{\alpha}$ in $\mathcal{H}^{0}$ using the calculus inequality (derived from Gagliardo-Nirenberg inequalities; cf. [46]):

$$
\left\|D^{\alpha}(f g)-f D^{\alpha} g\right\|_{\mathcal{H}^{0}} \leqq C\left(\|f\|_{\mathcal{H}^{s}}|g|_{L^{\infty}}+|\nabla f|_{L^{\infty}}\|g\|_{\mathcal{H}^{s-1}}\right)
$$

with the result that

$$
\left\|F_{\alpha}\right\|_{\mathcal{H}^{0}} \leqq C_{2}\left(\|\mathbf{v}\|_{\mathcal{H}^{s+1}}|\zeta|_{L^{\infty}}+|\nabla \mathbf{v}|_{L^{\infty}}\|\zeta\|_{\mathcal{H}^{s}}\right) .
$$

Since $|\zeta|_{L^{\infty}} \leqq|\nabla \mathbf{v}|_{L^{\infty}}+F|\psi|_{L^{\infty}}$, the bound on $\left\|F_{\alpha}\right\|_{\mathcal{H}^{0}}$ is simplified to

$$
\left\|F_{\alpha}\right\|_{\mathcal{H}^{0}} \leqq C_{3}\|\zeta\|_{\mathcal{H}^{s}}\left(\beta+|\nabla \mathbf{v}|_{L^{\infty}}+F|\psi|_{L^{\infty}}\right) .
$$

Multiplying Equation (6.43) by $\zeta_{\alpha}$ and integrating over the domain $\mathbf{T}^{2}$, we get $\frac{\mathrm{d}}{\mathrm{d} t}\|\zeta\|_{\mathcal{H}^{s}} \leqq\left\|F_{\alpha}\right\|_{\mathcal{H}^{0}}$, which implies

$$
\|\zeta\|_{\mathcal{H}^{s}} \leqq C_{4}\left\|\zeta^{I}\right\|_{\mathcal{H}^{s}} \exp \left\{C_{4} \int_{0}^{t}|\nabla \mathbf{v}|_{L^{\infty}} \mathrm{d} \tau\right\}
$$

where $C_{4}$ depends on $\beta$ and $T$, and the exponent contains $|\nabla \mathbf{v}|_{L^{\infty}}$ which needs to be estimated independently of time. The latter can be achieved with the help of an estimate analogous to the potential theory estimate for the Euler equation [58], which was derived for the quasi-geostrophic model by BourgeOuIs and BEALE [10], namely

$$
|\nabla \mathbf{v}|_{L^{\infty}} \leqq C_{5}|\zeta|_{L^{\infty}}\left(1+\ln ^{+} \frac{\|\zeta\|_{\mathcal{H}^{s}}}{|\zeta|_{L^{\infty}}}\right), \quad \ln ^{+} f=\left\{\begin{array}{c}
\ln f, f \geqq 1 \\
0, f<1
\end{array}\right.
$$

Taking into account that $|\zeta|_{L^{\infty}} \leqq C_{6}\left|\zeta^{I}\right|_{L^{\infty}} \leqq C_{6}\left\|\zeta^{I}\right\|_{\mathcal{H}^{s}}$, we get

$$
|\nabla \mathbf{v}|_{L^{\infty}} \leqq C_{7}\left\|\zeta^{I}\right\|_{\mathcal{H}^{s}}\left(1+\ln ^{+} \frac{\|\zeta\|_{\mathcal{H}^{s}}}{\left\|\zeta^{I}\right\|_{\mathcal{H}^{s}}}\right)
$$


substitution of which into (6.47) produces

$$
|\nabla \mathbf{v}|_{L^{\infty}} \leqq C_{8}\left\|\zeta^{I}\right\|_{\mathcal{H}^{s}}\left\{1+\int_{0}^{t}|\nabla \mathbf{v}|_{L^{\infty}} \mathrm{d} \tau\right\}
$$

Application of the integral Gronwall inequality yields

$$
|\nabla \mathbf{v}|_{L^{\infty}} \leqq C_{8}\left\|\zeta^{I}\right\|_{\mathcal{H}^{s}}\left(1+t C_{8}\left\|\zeta^{I}\right\|_{\mathcal{H}^{s}} \mathrm{e}^{t C_{8}\left\|\zeta^{I}\right\|_{\mathcal{H}^{s}}}\right)
$$

and therefore the function $Z(t)$ we sought is

$$
\|\zeta\|_{\mathcal{H}^{s}} \leqq C_{4}\left\|\zeta^{I}\right\|_{\mathcal{H}^{s}} \exp \left\{C_{4} \int_{0}^{t}|\nabla \mathbf{v}|_{L^{\infty}} \mathrm{d} \tau\right\} \equiv Z(t),
$$

where

$$
\int_{0}^{t}|\nabla \mathbf{v}|_{L^{\infty}} \mathrm{d} \tau \leqq C_{8}\left\|\zeta^{I}\right\|_{\mathcal{H}^{s}}\left\{(t+1)+(t-1) \mathrm{e}^{t C_{8}\left\|\zeta^{I}\right\|_{\mathcal{H}^{s}}}\right\}
$$

Based on this result and the local existence Theorem 4 we can prove the main global existence result.

Theorem 6. If initial data $\zeta^{I} \in \mathcal{H}^{s}, s \geqq 3$, then there exists a classical solution $\zeta \in$ $\widehat{\mathcal{C}}^{1}$ and $\psi \in \widehat{\mathcal{C}}_{0}^{2, \lambda}$, that is, in the space $X$ defined by (6.11), to the quasi-geostrophic equation (2.1), when $r=0$, on any finite time interval, $0 \leqq t \leqq T<\infty$.

Proof. From the local existence Theorem 4, it follows that there is a $T_{1}=T^{*}\left(\zeta_{0}\right)>$ 0 and a solution $\zeta \in \widehat{\mathcal{C}}^{1}\left[\left[0, T^{*}\right] \times \mathbf{T}^{2}\right]$. If $T_{1} \geqq T$, then we have the desired solution. If $T_{1}<T$, then define

$$
Z_{T}=\max _{0 \leqq t \leqq T} Z(t)
$$

Then $\left\|\zeta\left(T_{1}\right)\right\|_{\mathcal{H}^{s}}$ is bounded by $Z_{T}$ and thus $\left\|\zeta\left(T_{1}\right)\right\|_{\mathcal{C}^{1}}$ is bounded, too, since $\mathcal{H}^{s} \hookrightarrow \mathcal{C}^{1}$ for $s \geqq 3$. Thus we can again invoke the local existence Theorem 4 to continue the solution to time $T_{1}+T_{2}$. If $T_{1}+T_{2} \geqq T$, then the proof is completed. Otherwise, we continue this to $T_{1}+n T_{2}$ since $\left\|\bar{\zeta}\left(T_{1}+T_{2}\right)\right\|_{\mathcal{H}^{s}} \leqq Z_{T}$.

To prove that $\psi \in \widehat{\mathcal{C}}_{0}^{2, \lambda}$, note that by (6.1): $\zeta=M \psi$, where $M$ is an elliptic operator of the second order. Hence, one can apply the Schauder type estimate (5.20) to conclude that

$$
\|\psi\|_{\widehat{\mathcal{C}}_{0}^{3}} \leqq C\|\zeta\|_{\widehat{\mathcal{C}}_{0}^{1}}
$$

and thus trivially $\psi \in \widehat{\mathcal{C}}_{0}^{2, \lambda}$, as required. In the above argument we used the natural fact that $\zeta \in \widehat{\mathcal{C}}^{1}$ implies $\zeta \in \widehat{\mathcal{C}}_{0}^{1}$. 


\subsection{Global existence in the dissipative case}

The direct translation of the global estimates in the Hamiltonian case to the dissipative case is not available since, as discussed at the end of Section 6.2, the pointwise effect of dissipation remains unclear. Therefore, the global existence question is of separate interest; if a solution ceases to exist, this would imply that the model (2.1) is not adequate anymore and some new interesting physics takes place. However, for the purpose of proving dissipation-induced instability we do not actually need to demonstrate global existence in the dissipative case. The local existence result of Theorem 5 is enough to prove the strong Lyapunov instability as follows from Lemma 1 and Section 7. In the stable dissipative regime (below the critical point $U_{c} F / 2 \beta=2^{-1 / 2}(1+\sqrt{2})^{-1 / 2}$ on the dashed curve in Fig. 5), we actually do not need any information on the existence or stability of a solution in order to demonstrate the fact of dissipation-induced instability in the parameter range (2.14).

\section{Proof of Theorem 1 and discussion}

Proof. Based on Theorems 2-6, the main result-Theorem 1 on dissipation-induced instability in the quasi-geostrophic problem (2.1) — follows as a corollary, when all these theorems are applied in the class of classical solutions in the space $X$, given by (6.11):

- From Theorem 6 we know the existence of a classical solution of (2.1) in the Hamiltonian case, $r=0$, for all values of all parameters, that is, everywhere in Fig. 5.

- From Theorem 2 it follows that the equilibrium $\psi^{e}$ in the Hamiltonian case is Lyapunov stable for $U_{c} F / 2 \beta<1 / 2$, that is, below the solid curve in Fig. 5.

- From Theorem 3 and Lemma 1 and invoking the local existence result of Theorem 5 it follows that the equilibrium solution $\psi^{e}$ in the dissipative case is strongly Lyapunov unstable in $X$ for $2^{-1 / 2}(1+\sqrt{2})^{-1 / 2}<U_{c} F / 2 \beta<1 / 2$, that is, in the region between the dashed and solid curves in Fig. 5.

It should be noted that the dissipation-induced instability is proved in its strongest form; in particular, strong Lyapunov instability, that is when an unstable classical solution exists, is demonstrated.

In this paper we have presented a general approach for establishing dissipationinduced instabilities in infinite-dimensional systems and applied it in the case of the quasi-geostrophic system. In a nonlinear PDE context, the presence of a dissipation-induced instability phenomenon is proved rigorously for the first time. That is, the development is based on a nonlinear stability/instability proof, which is not conditional upon existence of a solution since the latter is demonstrated as well in a function space in which the nonlinear stability estimates are valid. The study demonstrates that a rigorous proof necessitates consideration of various interrelations between linear and nonlinear (in)stabilities and existence. It also 
reveals that current stability and existence methods are disjoint and suggests further developments of more efficient versions of these methods.

The observed phenomena were connected here to the geometrical picture known in the finite-dimensional case. Since the classification of forces is a nontrivial task in the infinite-dimensional case, especially in Eulerian variables and after various asymptotic approximations, used to derive (2.1), are applied, a consideration of the second variation $\delta^{2} H$, as in finite dimensions (1.3), seems to be the natural way of classifying the dissipation-induced instabilities in PDEs. As we established in this work, the dissipation-induced instability of quasi-geostrophic system (2.1) belongs to type (1.3b). We believe that the phenomenon proved for a two-layer model (2.1) in this work holds in various generalizations to the continuous stratification case and various boundary conditions, for example, an unbounded atmosphere in Jovian planets. Since our goal here is to demonstrate certain mathematical properties of the instability, but not to be utterly realistically close to actual atmosphere or ocean dynamics, we consider just the two-layer approximation of the quasi-geostrophic equations on a compact domain, which of course places a limit on the complexity of the vertical structure of the motion field, filtering out modes generally related to weaker instabilities [70]. In addition, the quasi-geostrophic equations themselves represent a parabolic approximation, which prohibits mixing across the tropopause (in the $z$-direction), while in reality the true dynamics is elliptic (cf. Pierrehumbert and SWAnson [74]).

While we discussed only one particular example, namely that of the baroclinic instability, one would expect that this phenomenon has wide appeal in various physical systems in analogy with finite-dimensional mechanical systems [48]. Indeed, infinite-dimensional dissipation-induced instabilities occur not only in fluid dynamics, but seem to be inherent in other field theories, for example general relativity [77] since the Einstein equations can be written in a form analogous to that of the NSEs, so that the dissipative effects become apparent [84].

In conclusion, this work is the first step in developing understanding of the dissipation-induced instability phenomena in infinite-dimensional systems. In general, the addition of dissipation may introduce higher order derivatives, as in the case of the NSEs, which modify both the equilibrium solution and the function space (in particular, boundary conditions: for example, slip versus no-slip) it belongs to. This is the major complication in the infinite-dimensional case with which we do not have to deal here in view of the particularity of our model: the form of dissipation does not increase the order of Equation (2.1) and leaves the equilibrium solution and the function space unaltered. In the fluid dynamics context, the destabilizing effect of viscosity, which is obviously responsible for dissipation, has been noticed a long time ago, cf. LIN [55]. Namely, base state velocity profiles without inflection points are known to be stable in the inviscid case according to Rayleigh's theorem, while in the viscous case the same velocity profiles may become unstable, for example in the case of the Poiseuille flow. Hence, explaining the destabilizing effect of viscosity, when the equilibrium solution (the basic state in fluid mechanics terminology) is modified, is not a trivial problem alone: even transition to turbulence in a simple geometry such as a channel or a pipe (Couette, Poiseuille, HagenPoiseuille flows) is still an unresolved challenge [49]. The general phenomena we 
studied here embrace those long-standing questions as well. However, in order to attack those problems one would need to extend the function-analytic framework of dissipation-induced instabilities to the situation when dissipation modifies the equilibrium solution, as it happens in the NSEs, for example by either relaxing the requirement that the equilibrium solutions should be the same in the conservative and dissipative cases or by considering the conservative base state as a weakly convergent limit of the dissipative one when dissipation vanishes.

Acknowledgments. The authors would like to thank Tapio Schneider, John Hart, Steve Shkoller, and Edriss Titi for helpful discussions. The authors were partially supported by NSF-ITR Grant ACI-0204932. R.K. also acknowledges partial support from NSERC 341849-2007.

\section{Appendix A: The origin of the quasi-geostrophic model}

In this Appendix we provide a brief self-contained derivation of the model (2.1) to highlight the physical importance and the origin of various effects, and discuss the properties relevant to our study. The reader interested in an alternative derivation (namely, as a limit from compressible case) and the general context of this problem, is referred to PEDLOSKY [70].

The physical setup is given in Fig. 3 showing a working domain, located on the surface of a rotating planet. This domain is a rectangular channel of finite depth $D$ in the direction $z$ perpendicular to the Earth's surface, and with finite dimensions both in the west-east (zonal) periodic direction $x$ and in the north-south (meridional) bounded by solid walls direction $y$. Without any influence on the stability results we regard it as a channel of dimension $L$ in the $x$-direction and of dimension $L / 2$ in the $y$-direction.

The quasi-geostrophic model in the two-layer viscous beta-plane approximation is derived from the general momentum equations for viscous incompressible fluid (the NSEs) written for an observer in a uniformly rotating coordinate frame,

$$
\rho\left[\mathrm{d}_{t} \mathbf{v}+2 \boldsymbol{\Omega} \times \mathbf{v}\right]=-\nabla p+\rho \nabla \Phi+\mu \nabla^{2} \mathbf{v}
$$

where $\mathrm{d}_{t}$ stands for the total three-dimensional (material) derivative, $\mathbf{v}$ is the velocity as seen in a rotating frame of reference, and $\Phi=\phi+\phi_{c}$ is the geopotential, where $\phi_{c}=|\boldsymbol{\Omega} \times \mathbf{r}|^{2} / 2$ is a potential of centripetal acceleration. The stratification is modeled by two superimposed layers with interface $h(t, x, y)$, located halfway between the horizontal planes with index 1 referring to the upper layer and 2 to the lower one (cf. Fig. 3), at which the kinematic boundary condition is given by

$$
z=h(t, x, y): \quad h_{t}+u h_{x}+v h_{y}=w,
$$

where $w$ is the $z$-component of velocity. The effect of the Earth's sphericity is modeled by $\beta$-plane approximation, $2 \Omega=f_{0}+\beta^{\prime} y$ (the $y$-dependence models 
the variation of the Earth's Coriolis effect with latitude: see RossBy [76]). If the characteristic velocity scale in the $(x, y)$-plane is $U$, then the scaling

$$
\begin{aligned}
(x, y) & \rightarrow L(x, y), z \rightarrow D z,(u, v) \rightarrow U(u, v), w \rightarrow U(D / L) w, \\
t & \rightarrow \frac{L}{U} t, h \rightarrow \frac{D}{2}+\frac{\rho_{1} U L f_{0}}{g\left(\rho_{2}-\rho_{1}\right)} h,
\end{aligned}
$$

with pressure redefined to eliminate the hydrostatic component according to $p_{1} \rightarrow(D-z) \rho_{1} g+\rho_{1} U f_{0} L p_{1}, p_{2} \rightarrow \frac{1}{2} D \rho_{1} g+\left(\frac{1}{2} D-z\right) \rho_{2} g+\rho_{2} U f_{0} L p_{2}$,

reduces the problem to a set of momentum equations posed on the domain $\mathbf{D}=\{-1 \leqq x \leqq 1 ; 0 \leqq y \leqq 1\}$ in each layer (we omit layer indexes for brevity)

$$
\begin{aligned}
\epsilon \mathrm{d}_{t} u-(1+\epsilon \beta y) v & =-p_{x}+\frac{1}{2} E \Delta_{\delta} u, \\
\epsilon \mathrm{d}_{t} v+(1+\epsilon \beta y) u & =-p_{y}+\frac{1}{2} E \Delta_{\delta} v, \\
\epsilon \delta^{2} \mathrm{~d}_{t} w & =-p_{z}+\frac{1}{2} E \delta^{2} \Delta_{\delta} w, \\
u_{x}+v_{y}+w_{z} & =0,
\end{aligned}
$$

along with the kinematic boundary condition

$$
z=\frac{1}{2}+\frac{1}{2} \epsilon F h(t, x, y): \quad \frac{1}{2} \epsilon F\left[h_{t}+u h_{x}+v h_{y}\right]=w .
$$

Here the nondimensional parameters, namely the aspect ratio $\delta$, the Rossby number $\epsilon$, Eckman number $E$, internal rotational Froude number $F$, planetary vorticity factor $\beta$ are defined as

$$
\delta=\frac{D}{L}, \quad \epsilon=\frac{U}{f_{0} L}, \quad E=\frac{2 v}{f_{0} D^{2}}, \quad F=f_{0}^{2} L^{2}\left[g \frac{\Delta \rho}{\rho_{1}} \frac{D}{2}\right]^{-1}, \quad \beta=\beta^{\prime} \frac{L^{2}}{U} .
$$

The scaled Laplacian is defined by $\Delta_{\delta}=\delta^{2}\left(\partial_{x}^{2}+\partial_{y}^{2}\right)+\partial_{z}^{2}$. The quasi-geostrophic approximation results from the following assumptions on the above parameters:

$$
(\epsilon, E, \Delta \rho / \rho, \delta) \ll 1,(\beta, F) \sim O(1), \epsilon \ll \mathrm{e}^{1 / 2}
$$

which suggests the low Rossby number expansion for the solution vector $\mathbf{U}=$ $(u, v, w, h, p): \mathbf{U}=\mathbf{U}^{(0)}+\epsilon \mathbf{U}^{(1)}+O\left(\epsilon^{2}\right)$, where $w^{(0)}=0$. This yields the zero-order approximation,

$$
v^{(0)}=p_{x}^{(0)}, \quad u^{(0)}=-p_{y}^{(0)}, \quad 0=-p_{z}^{(0)}, \quad u_{x}^{(0)}+v_{y}^{(0)}=0,
$$

where the first two equations are a geostrophic approximation (balance of Coriolis and pressure forces) independent of $z$, while the third equation is a hydrostatic approximation. The order one approximation is given by

$$
\begin{aligned}
\mathrm{d}_{t}^{0} u^{(0)}-v^{(1)}-\beta y v^{(0)} & =-p_{x}^{(1)}, \\
\mathrm{d}_{t}^{0} v^{(0)}+u^{(1)}+\beta y u^{(0)} & =-p_{y}^{(1)}, \\
0 & =-p_{z}^{(1)}, \\
u_{x}^{(1)}+v_{y}^{(1)}+w_{z}^{(1)} & =0,
\end{aligned}
$$


with kinematic boundary condition at $z=\frac{1}{2}$ given by $\frac{1}{2} F \mathrm{~d}_{t}^{0} h^{(0)}=w^{(1)}$, where the material derivative is now two-dimensional: $\mathrm{d}_{t}^{0}=\partial_{t}+u^{(0)} \partial_{x}+v^{(0)} \partial_{y}$. Eliminating pressure from the $x$ - and $y$-momentum equations, we arrive at

$$
\mathrm{d}_{t}^{0} \zeta^{(0)}=w_{z}^{(1)}+\beta v^{(0)}, \quad \zeta^{(0)}=v_{x}^{(0)}-u_{y}^{(0)}=\Delta p^{(0)}, \quad \Delta=\partial_{x}^{2}+\partial_{y}^{2} .
$$

Since the left-hand side of the equation for $\mathrm{d}_{t}^{0} \zeta^{(0)}$ is independent of $z$, we can integrate across each layer and obtain, with the use of the kinematic conditions for layer 1 and 2, respectively,

$$
\begin{aligned}
& \mathrm{d}_{t}^{0} \zeta^{(0)}+F \mathrm{~d}_{0} h^{(0)}=\left.2 w^{(1)}\right|_{z=1}-\beta v^{(0)} \\
& \mathrm{d}_{t}^{0} \zeta^{(0)}-F \mathrm{~d}_{0} h^{(0)}=-\left.2 w^{(1)}\right|_{z=0}-\beta v^{(0)}
\end{aligned}
$$

Next, the dynamic condition at the interface, $z=h$, reads in dimensional variables as $p_{1}=p_{2}$, or, after simplifications,

$$
p_{2}^{(0)}-p_{1}^{(0)}=h^{(0)}+O(\Delta \rho / \rho),
$$

where viscous stresses are neglected. It is convenient to introduce the streamfunction $\psi^{(0)}=p^{(0)}$, which in this case coincides with the pressure; the velocity components are defined respectively as $v^{(0)}=\psi_{x}^{(0)}$ and $u^{(0)}=-\psi_{y}^{(0)}$. Thus the intermediate form of the quasi-geostrophic equations is

$$
\begin{aligned}
& \mathrm{d}_{t}^{0}\left[\zeta^{(0)}+F\left(\psi_{2}^{(0)}-\psi_{1}^{(0)}\right)+\beta y\right]=\left.2 w^{(1)}\right|_{z=1}, \\
& \mathrm{~d}_{t}^{0}\left[\zeta^{(0)}-F\left(\psi_{2}^{(0)}-\psi_{1}^{(0)}\right)+\beta y\right]=-\left.2 w^{(1)}\right|_{z=0},
\end{aligned}
$$

where the meaning of the right-hand side needs to be explained. The form of the right-hand side of Equations (A.12) suggests that the fluid is pumped through the boundaries and thus makes the equations nonconservative - this origin of the dissipation was uncovered by CHARNEY and ELIASSEN [14]. Physically this corresponds to a fine structure of the boundary layers-Eckman layers - which can be derived from (A.3) by the simple re-scaling $z \rightarrow l z^{\prime}$ and $w \rightarrow l w^{\prime}$, while keeping the leading order approximation

$$
-v=-p_{x}+\frac{1}{2} u_{z^{\prime} z^{\prime}}, \quad u=-p_{y}+\frac{1}{2} v_{z^{\prime} z^{\prime}}, \quad 0=-p_{z^{\prime}},
$$

with $l=\mathrm{e}^{1 / 2}$. Next, using the fact that outside the boundary layer $\bar{v}=p_{x}$ and $\bar{u}=-p_{y}$, and utilizing the no-slip boundary condition, $z^{\prime}=0: u=v=w^{\prime}=0$, we can integrate these equations to find the general expression for $w^{\prime}$ :

$$
w^{\prime}=\frac{1}{2}\left(\bar{v}_{x}-\bar{u}_{y}\right)\left[1-\mathrm{e}^{-z^{\prime}}\left(\cos z^{\prime}+\sin z^{\prime}\right)\right] .
$$

Taking the limit $z^{\prime} \rightarrow+\infty$, which corresponds to $z \rightarrow 0$ in an asymptotic sense, we find that $\left.w^{(1)}\right|_{z=0}=\frac{\mathrm{e}^{1 / 2}}{2 \epsilon} \zeta^{(0)}$, and similarly $\left.w^{(1)}\right|_{z=1}=-\frac{\mathrm{e}^{1 / 2}}{2 \epsilon} \zeta^{(0)}$ (the latter 
is obtained from the boundary layer near $z=1$ by transforming $\left.z \rightarrow\left(1-z^{\prime}\right) / l\right)$. Therefore, the final quasi-geostrophic equations are

$$
\begin{aligned}
& \mathrm{d}_{t}^{0}\left[\zeta^{(0)}+F\left(\psi_{2}^{(0)}-\psi_{1}^{(0)}\right)+\beta y\right]=-r \Delta \psi_{1}, \\
& \mathrm{~d}_{t}^{0}\left[\zeta^{(0)}-F\left(\psi_{2}^{(0)}-\psi_{1}^{(0)}\right)+\beta y\right]=-r \Delta \psi_{2},
\end{aligned}
$$

which after the introduction of the potential vorticity defined by

$$
q_{i}=\nabla^{2} \psi_{i}+(-1)^{i} F\left(\psi_{1}-\psi_{2}\right)+\beta y,
$$

and dropping off indexes yields the differential formulation (2.1). Thus, in addition to the nondimensional parameters (A.5) there is one more responsible for dissipations effects, $r=\mathrm{e}^{1 / 2} / \epsilon$. As discussed in PeDLOSKY [70], this particular dissipation acts like a sink of potential vorticity. The distinctive feature of the dissipation term, $-r \Delta \psi_{i}$, is that it does not increase the order of the PDEs as opposed to the Navier-Stokes type of dissipation - this allows one to study the stability of the same equilibrium solution in both the conservative and dissipative formulations. We make use of this interpretation in Section 5.

Since the channel is periodic in $x$, the appropriate boundary conditions correspond to $D^{\alpha} \psi_{i}(x, y),|\alpha| \geqq 0$ being periodic with period 2 , and since the channel is bounded by walls at $y=0,1$, a no-penetration condition applies,

$$
y=0,1: v=\psi_{x}=0,
$$

which implies that the stream-function is only a function of time at solid walls. Applying the first order $x$-momentum equation to the boundaries $y=0,1$ we find: $u_{t}^{(0)}+u^{(0)} u_{x}^{(0)}=-p_{x}^{(1)}$, which after integration with respect to $x$ yields another boundary condition, first established by PHILliPs [72]:

$$
y=0,1: \frac{\partial}{\partial t} \Gamma_{i}(y)=0, \quad \Gamma_{i}(y)=\int_{-1}^{1} u_{i}^{(0)} \mathrm{d} x,
$$

where we have exploited the periodic boundary conditions. The latter boundary conditions guarantee the uniqueness of the solution (otherwise the no-penetration condition is satisfied by an arbitrary function of time $t$ ), and can be loosely interpreted as the conservation of circulations $\Gamma_{i}(y)$ at the solid boundaries.

\section{Appendix B: Particle path estimates (6.20)}

To justify (6.20a) take Equation (6.17) and utilize the velocity bound (6.16):

$$
\left|\mathrm{d}_{\tau}\|\xi\|_{\mathcal{C}^{1, \lambda}}\right|=\|u\|_{\mathcal{C}^{1, \lambda}} \leqq\left. K_{3}(1+\epsilon) \Longrightarrow\|\xi\|_{\mathcal{C}^{1, \lambda}}\right|_{\tau=0} ^{\tau=t} \leqq K_{3}(1+\epsilon) t
$$

so that with the use of the obvious inequality (cf. AdAms [2])

$$
\|\xi\|_{\mathcal{C}^{0}}(t) \leqq\|\xi\|_{\mathcal{C}^{1, \lambda}}(t) \leqq K_{3}(1+\epsilon) t+\left\|\left.\xi\right|_{\tau=0}\right\|_{\mathcal{C}^{1, \lambda}}
$$


Now taking the supremum over $0 \leqq t \leqq T$ and taking into account the size of the domain $\mathbf{T}^{2}$ which results in $\max \left\|\left.\bar{\xi}\right|_{\tau=0}\right\|_{\mathcal{C}^{1, \lambda}}=1$, we finally get estimate (6.20a):

$$
\|\xi\|_{\widehat{\mathcal{C}}_{0}^{0}} \leqq K_{3}(1+\epsilon) T+1 .
$$

In order to get estimate (6.20b) let us apply the gradient $\nabla_{\mathbf{x}}$ to Equations (6.17),

$$
\frac{\mathrm{d}}{\mathrm{d} \tau} \nabla \xi=\frac{\mathrm{d}}{\mathrm{d} \tau}\left(\begin{array}{c}
\nabla \xi \\
\nabla \eta
\end{array}\right)=\left(\begin{array}{c}
\nabla u \\
\nabla v
\end{array}\right)=\left(\begin{array}{cc}
u_{x} & u_{y} \\
v_{x} & v_{y}
\end{array}\right)=\left(\begin{array}{cc}
u_{\xi} & u_{\eta} \\
v_{\xi} & v_{\eta}
\end{array}\right)\left(\begin{array}{ll}
\xi_{x} & \xi_{y} \\
\eta_{x} & \eta_{y}
\end{array}\right),
$$

which yields

$$
\begin{aligned}
\left\|\frac{\mathrm{d}}{\mathrm{d} \tau}\left(\begin{array}{ll}
\xi_{x} & \xi_{y} \\
\eta_{x} & \eta_{y}
\end{array}\right)\right\|_{\mathcal{C}^{0}} & =\left\|\left(\begin{array}{ll}
u_{\xi} & u_{\eta} \\
v_{\xi} & v_{\eta}
\end{array}\right)\left(\begin{array}{ll}
\xi_{x} & \xi_{y} \\
\eta_{x} & \eta_{y}
\end{array}\right)\right\|_{\mathcal{C}^{0}} \\
& \leqq 2\left\|\left(\begin{array}{ll}
u_{\xi} & u_{\eta} \\
v_{\xi} & v_{\eta}
\end{array}\right)\right\|_{\mathcal{C}^{0}}\left\|\left(\begin{array}{ll}
\xi_{x} & \xi_{y} \\
\eta_{x} & \eta_{y}
\end{array}\right)\right\|_{\mathcal{C}^{0}},
\end{aligned}
$$

so that using the inequality $\left\|\nabla_{\boldsymbol{\xi}} \mathbf{v}\right\|_{\mathcal{C}^{0}} \leqq\left\|\nabla_{\boldsymbol{\xi}} \mathbf{v}\right\|_{\mathcal{C}^{0, \lambda}}$ together with the obvious implication

$$
\|\mathbf{v}\|_{\mathcal{C}^{1, \lambda}} \leqq K_{3}(1+\epsilon) \Rightarrow\left\|\nabla_{\xi} \mathbf{v}\right\|_{\mathcal{C}^{0, \lambda}} \leqq K_{3}(1+\epsilon),
$$

and going back to vector notation, we get

$$
\left|\mathrm{d}_{\tau}\left\|\nabla_{\mathbf{x}} \boldsymbol{\xi}\right\|_{\mathcal{C}^{0}}\right| \leqq 2\left\|\nabla_{\boldsymbol{\xi}} \mathbf{v}\right\|_{\mathcal{C}^{0, \lambda}}\left\|\nabla_{\mathbf{x}} \xi\right\|_{\mathcal{C}^{0}} \Rightarrow\left\|\nabla_{\mathbf{x}} \xi\right\|_{\mathcal{C}^{0}}(t) \leqq \mathrm{e}^{2 K_{3}(1+\epsilon) t},
$$

where (6.16) was used. Taking the supremum over $0 \leqq t \leqq T$ furnishes (6.20b),

$$
\left\|\nabla_{\mathbf{x}} \xi\right\|_{\widehat{\mathcal{C}}_{0}^{0}}(t) \leqq \mathrm{e}^{2 K_{3}(1+\epsilon) T} .
$$

Next, to get bound $(6.20 \mathrm{c})$, again apply gradient $\nabla_{\mathbf{x}}$ to $(6.17)$ and formulate a difference,

$$
\begin{aligned}
\mathrm{d}_{\tau}\left\{\nabla_{\mathbf{x}^{\prime}} \xi^{\prime}-\nabla_{\mathbf{x}^{\prime \prime}} \xi^{\prime \prime}\right\} & =\nabla_{\xi^{\prime}} \mathbf{v} \nabla_{\mathbf{x}^{\prime}} \xi^{\prime}-\nabla_{\xi^{\prime \prime}} \mathbf{v} \nabla_{\mathbf{x}^{\prime \prime}} \boldsymbol{\xi}^{\prime \prime} \\
& =\left(\nabla_{\xi^{\prime}} \mathbf{v}-\nabla_{\xi^{\prime \prime}} \mathbf{v}\right) \nabla_{\mathbf{x}^{\prime}} \xi^{\prime}+\nabla_{\xi^{\prime \prime}} \mathbf{v}\left(\nabla_{\mathbf{x}^{\prime}} \boldsymbol{\xi}^{\prime}-\nabla_{\mathbf{x}^{\prime \prime}} \xi^{\prime \prime}\right) .
\end{aligned}
$$

Dividing this expression by $\left|\mathbf{x}^{\prime}-\mathbf{x}^{\prime \prime}\right|^{\lambda}$ and recalling definition (2.5) and that $\|\nabla \mathbf{v}\|_{\mathcal{C}^{0}} \leqq\|\mathbf{v}\|_{\mathcal{C}^{1, \lambda}}$, we find

$$
\begin{aligned}
\left|\frac{\mathrm{d}}{\mathrm{d} \tau}[\nabla \boldsymbol{\xi}]_{\mathcal{C}^{0, \lambda}}\right| & \leqq\|\mathbf{v}\|_{\mathcal{C}^{1}}[\nabla \xi]_{\mathcal{C}^{0, \lambda}}+\left|\sup _{\mathbf{x}^{\prime}, \mathbf{x}^{\prime \prime}} \frac{\nabla_{\xi^{\prime}} \mathbf{v}-\nabla_{\xi^{\prime \prime}} \mathbf{v}}{\left|\xi^{\prime}-\xi^{\prime \prime}\right|^{\lambda}-\left.\xi^{\prime \prime}\right|^{\lambda}} \nabla_{\mathbf{x}^{\prime}} \xi^{\prime}\right| \\
& \leqq\|\mathbf{v}\|_{\mathcal{C}^{1}}[\nabla \xi]_{\mathcal{C}^{0, \lambda}}+\left.\|\mathbf{v}\|_{\mathcal{C}^{1, \lambda}}\left(\|\nabla \boldsymbol{\xi}\|_{\mathcal{C}^{0}}\right)^{1+\lambda}\right|^{\lambda} \\
& \leqq K_{3}(1+\epsilon) \mathrm{e}^{2 K_{3}(1+\epsilon)(1+\lambda) T}+K_{3}(1+\epsilon)[\nabla \xi]_{\mathcal{C}^{0, \lambda}}
\end{aligned}
$$

Since $\nabla \xi=I$ at $\tau=t$ and thus $[\nabla \xi]_{\mathcal{C}^{0, \lambda}}=0$ for $\tau=t$, then integration of the previous expression yields

$$
\left.[\nabla \xi]_{\mathcal{C}^{0, \lambda}}\right|_{\tau=0} \leqq \mathrm{e}^{2 K_{3}(1+\lambda)(1+\epsilon) T}\left(\mathrm{e}^{K_{3}(1+\epsilon) t}-1\right)
$$


and taking the supremum over $0 \leqq t \leqq T$ produces $(6.20 \mathrm{c})$

$$
[\nabla \xi]_{\widehat{\mathcal{C}}_{0}^{0, \lambda}} \leqq \mathrm{e}^{2 K_{3}(1+\lambda)(1+\epsilon) T}\left(\mathrm{e}^{K_{3}(1+\epsilon) T}-1\right) \leqq \mathrm{e}^{K_{3}(3+2 \lambda)(1+\epsilon) T}
$$

To validate estimate (6.20d) we appeal to Equations (6.21) and use estimates (6.16, 6.20b):

$$
\begin{aligned}
\left\|\partial_{t} \boldsymbol{\xi}\right\|_{\widehat{\mathcal{C}}_{0}^{0}} \leqq\left\|\mathbf{v} \cdot \nabla_{\mathbf{x}} \boldsymbol{\xi}\right\|_{\widehat{\mathcal{C}}_{0}^{0}} \leqq\|\mathbf{v}\|_{\widehat{\mathcal{C}}_{0}^{0}}\left\|\nabla_{\mathbf{x}} \boldsymbol{\xi}\right\|_{\widehat{\mathcal{C}}_{0}^{0}} \\
\\
\qquad\|\mathbf{v}\|_{\widehat{\mathcal{C}}_{0} 1, \lambda}\left\|\nabla_{\mathbf{x}} \xi\right\|_{\widehat{\mathcal{C}}_{0}^{0}} \leqq K_{3}(1+\epsilon) \mathrm{e}^{2 K_{3}(1+\epsilon) T},
\end{aligned}
$$

which is $(6.20 \mathrm{~d})$. The same procedure is applicable to get bound $(6.20 \mathrm{e})$, that is, using (6.20b-6.20c) we arrive at

$$
\begin{aligned}
{\left[\frac{\partial \xi}{\partial t}\right]_{\widehat{\mathcal{C}}_{0}^{0, \lambda}} } & \leqq\left[\mathbf{v} \cdot \nabla_{\mathbf{x}} \xi\right]_{\widehat{\mathcal{C}}_{0}^{0, \lambda}}=\sup _{\mathbf{x}^{\prime}, \mathbf{x}^{\prime \prime}} \frac{\mathbf{v}\left(\boldsymbol{\xi}^{\prime}\right) \nabla_{\mathbf{x}^{\prime}} \boldsymbol{\xi}^{\prime}-\mathbf{v}\left(\boldsymbol{\xi}^{\prime \prime}\right) \nabla_{\mathbf{x}^{\prime \prime}} \boldsymbol{\xi}^{\prime \prime}}{\left|\xi^{\prime}-\xi^{\prime \prime}\right|^{\lambda}} \\
& =\sup _{\mathbf{x}^{\prime}, \mathbf{x}^{\prime \prime}} \frac{\left(\mathbf{v}\left(\xi^{\prime}\right)-\mathbf{v}\left(\boldsymbol{\xi}^{\prime \prime}\right)\right) \nabla_{\mathbf{x}^{\prime}}+\mathbf{v}\left(\boldsymbol{\xi}^{\prime \prime}\right)\left(\nabla_{\mathbf{x}^{\prime}} \boldsymbol{\xi}^{\prime}-\nabla_{\mathbf{x}^{\prime \prime}} \boldsymbol{\xi}^{\prime \prime}\right)}{\left|\boldsymbol{\xi}^{\prime}-\xi^{\prime \prime}\right|^{\lambda}} \\
& \leqq 2\|\mathbf{v}\|_{\widehat{\mathcal{C}}_{0}^{1, \lambda}}\left[\nabla_{\mathbf{x}} \xi\right]_{\widehat{\mathcal{C}}_{0}^{0, \lambda}} \leqq 2 K_{3}(1+\epsilon) \mathrm{e}^{K_{3}(3+2 \lambda)(1+\epsilon) T}
\end{aligned}
$$

which is correct but quite conservative.

\section{Appendix C: Vorticity estimates (6.23)}

Now we turn our attention to the solution (6.22) of the initial value problem (6.2). As we know, $\xi \in \widehat{\mathcal{C}}_{0}^{1, \lambda}$ and $\zeta^{I} \in \mathcal{C}^{1}$. Therefore, it follows that $\zeta \in \mathcal{C}^{1}$. To establish bound (6.23a), we use Equation (6.22),

$$
\begin{aligned}
\|\widetilde{\zeta}\|_{\mathcal{C}^{0}}=\left\|\zeta^{I}+\beta(\eta-y)\right\|_{\mathcal{C}^{0}} & \leqq\left\|\zeta^{I}\right\|_{\mathcal{C}^{0}}+\|\beta(\eta-y)\|_{\mathcal{C}^{0}} \\
& \leqq\left\|\zeta^{I}\right\|_{\mathcal{C}^{1}}+\left|\sup _{\mathbf{x} \in \mathbf{T}^{2}} \beta(\eta-y)\right| \leqq\left\|\zeta^{I}\right\|_{\mathcal{C}^{1}}+2 \beta
\end{aligned}
$$

Now, taking the supremum over $0 \leqq t \leqq T$ we get

$$
\|\widetilde{\zeta}\|_{\widehat{\mathcal{C}}_{0}^{0}} \leqq\left\|\zeta^{I}\right\|_{\mathcal{C}^{1}}+2 \beta
$$

Applying the gradient to (6.22) gives

$$
\nabla_{\mathbf{x}} \widetilde{\zeta}=\nabla_{\mathbf{x}} \zeta^{I}+\beta[\nabla \eta-(0,1)]=\left[\nabla_{\xi} \zeta^{I}+\beta(0,1)\right]\left(\begin{array}{ll}
\xi_{x} & \xi_{y} \\
\eta_{x} & \eta_{y}
\end{array}\right)-\beta(0,1),
$$

so that

$$
\left\|\nabla_{\mathbf{x}} \widetilde{\zeta}\right\|_{\widehat{\mathcal{C}}_{0}^{0}} \leqq\left\{\left\|\zeta^{I}\right\|_{\mathcal{C}^{1}}+\beta\right\} \mathrm{e}^{2 K_{3}(1+\epsilon) T}+\beta
$$


where we used (6.20b). Combining expressions (C.1-C.2), we find

$$
\|\widetilde{\zeta}\|_{\widehat{\mathcal{C}}_{0}^{1}} \leqq\|\widetilde{\zeta}\|_{\widehat{\mathcal{C}}_{0}^{0}}+\left\|\nabla_{\mathbf{x}} \widetilde{\zeta}\right\|_{\widehat{\mathcal{C}}_{0}^{0}} \leqq L_{1} \mathrm{e}^{2 K_{3}(1+\epsilon) T}, \quad L_{1}=2\left\|\zeta^{I}\right\|_{\mathcal{C}^{1}}+4 \beta,
$$

that is, (6.23a). Finally, differentiating (6.22) with respect to time $t$ :

$$
\partial_{t} \widetilde{\zeta}=\nabla_{\xi} \widetilde{\zeta}^{I} \cdot \partial_{t} \xi+\beta \partial_{t} \eta
$$

so that

$$
\left\|\partial_{t} \widetilde{\zeta}\right\|_{\mathcal{C}^{0}} \leqq\left\|\widetilde{\zeta}^{I}\right\|_{\mathcal{C}^{1}}\left\|\partial_{t} \boldsymbol{\xi}\right\|_{\mathcal{C}^{0}}+\beta\left\|\partial_{t} \eta\right\|_{\mathcal{C}^{0}}
$$

and taking the supremum over $0 \leqq t \leqq T$ we recover $(6.23 \mathrm{~b})$ :

$$
\left\|\partial_{t} \widetilde{\zeta}\right\|_{\widehat{\mathcal{C}}_{0}^{0}} \leqq\left\{\left\|\widetilde{\zeta}^{I}\right\|_{\mathcal{C}^{1}}+\beta\right\} K_{3}(1+\epsilon) \mathrm{e}^{2 K_{3}(1+\epsilon) T} \leqq L_{1} K_{3}(1+\epsilon) \mathrm{e}^{2 K_{3}(1+\epsilon) T}
$$

where $L_{1}$ is the same as above and the resulting estimate is more conservative than it needs to be.

\section{Appendix D: Vorticity estimates with dissipation (6.34)}

The solution of the problem with dissipation, that is, (6.31), is given by $\widetilde{\zeta}_{i}=\mathrm{e}^{-r t} \widehat{\zeta}_{i}$, where

$$
\begin{aligned}
\widehat{\zeta}(x, y, t)= & \widehat{\zeta}^{I}(\xi(0 ; x, y, t), \eta(0 ; x, y, t))+\beta\left[\eta(0 ; x, y, t)-\mathrm{e}^{r t} y\right] \\
& +r \int_{0}^{t}\left\{\beta \eta(\tau ; x, y, t)+F(-1)^{i}\left[\widetilde{\psi}_{1}-\widetilde{\psi}_{2}\right](\xi(\tau ; x, y, t), \tau)\right\} \mathrm{e}^{r \tau} \mathrm{d} \tau .
\end{aligned}
$$

Let $\{\ldots\}=\int_{0}^{t}\left\{\beta \eta(\tau ; x, y, t)+F(-1)^{i}\left[\widetilde{\psi}_{1}-\widetilde{\psi}_{2}\right](\xi(\tau ; x, y, t), \tau)\right\} \mathrm{e}^{r \tau} \mathrm{d} \tau$. First, note that

$$
\begin{aligned}
\|\widetilde{\zeta}\|_{\mathcal{C}^{s}} & =\left\|\widehat{\zeta}^{I} \mathrm{e}^{-r t}+\beta\left(\eta \mathrm{e}^{-r t}-y\right)+r \mathrm{e}^{-r t}\{\ldots\}\right\|_{\mathcal{C}^{s}} \\
& \leqq\left\|\widehat{\zeta}^{I} \mathrm{e}^{-r t}\right\|_{\mathcal{C}^{s}}+\left\|\beta\left(\eta \mathrm{e}^{-r t}-y\right)\right\|_{\mathcal{C}^{s}}+\left\|r \mathrm{e}^{-r t}\{\ldots\}\right\|_{\mathcal{C}^{s}} \\
& \leqq\left\|\widehat{\zeta}^{I} \mathrm{e}^{-r t}\right\|_{\mathcal{C}^{s+1}}+\left|\sup _{\mathbf{x} \in \mathbf{T}^{2}} \beta\left(\eta \mathrm{e}^{-r t}-y\right)\right|+r\left\|\mathrm{e}^{-r t}\{\ldots\}\right\|_{\mathcal{C}^{s}}
\end{aligned}
$$

Applying sup to both sides we get $0 \leqq t \leqq T$

$$
\begin{aligned}
\|\widetilde{\zeta}\|_{\widehat{\mathcal{C}}^{s}} & \leqq\left\|\widehat{\zeta}^{I}\right\|_{\widehat{\mathcal{C}}^{s+1}}+2 \beta+\sup _{0 \leqq t \leqq T} r \mathrm{e}^{-r t}\|\{\ldots\}\|_{\mathcal{C}^{s}} \\
& =\left\|\widehat{\zeta}^{I}\right\|_{\widehat{\mathcal{C}}^{s+1}}+2 \beta+r T \mathrm{e}^{r T}\left\{\beta+2 F K_{3}(1+\epsilon)\right\} .
\end{aligned}
$$


Now,

$$
\begin{aligned}
\|\{\ldots\}\|_{\mathcal{C}^{s}} & \leqq \beta\left\|\int_{0}^{t} \eta(\tau ; x, y, t) \mathrm{d} \tau\right\|_{\mathcal{C}^{s}}+F\left\|\int_{0}^{t}\left(\widetilde{\psi}_{1}-\widetilde{\psi}_{2}\right) \mathrm{e}^{r \tau} \mathrm{d} \tau\right\|_{\mathcal{C}^{s}} \\
& \leqq \beta \mathrm{e}^{r T} T+2 F K_{3}(1+\epsilon) \mathrm{e}^{r T} T
\end{aligned}
$$

as follows from (6.14) and the embedding theorem [2]. Next, applying the gradient $\nabla_{\mathbf{x}}$ to $(6.33)$ gives

$$
\begin{aligned}
\nabla_{\mathbf{x}} \widetilde{\zeta}= & \nabla_{\mathbf{x}} \mathrm{e}^{-r t}\left\{\widehat{\zeta}^{I}+\beta\left(\eta-\mathrm{e}^{r t} y\right)\right\}+r \mathrm{e}^{-r t} \beta \int_{0}^{t}(0,1)\left(\begin{array}{ll}
\xi_{x} & \xi_{y} \\
\eta_{x} & \eta_{y}
\end{array}\right) \mathrm{e}^{r \tau} \mathrm{d} \tau \\
& +r \mathrm{e}^{-r t}(-1)^{i} F \int_{0}^{t} \mathrm{e}^{r \tau} \nabla_{\xi}\left(\psi_{1}-\psi_{2}\right)\left(\begin{array}{ll}
\xi_{x} & \xi_{y} \\
\eta_{x} & \eta_{y}
\end{array}\right) \mathrm{d} \tau
\end{aligned}
$$

and thus, using (6.16) for $\nabla_{\xi}\left(\psi_{1}-\psi_{2}\right)$, we get

$$
\begin{aligned}
\left\|\nabla_{\mathbf{x}} \widetilde{\zeta}\right\|_{\widehat{\mathcal{C}}^{s}} \leqq & \left\{\left\|\zeta^{I}\right\|_{\mathcal{C}^{s+1}}+\beta\right\} \mathrm{e}^{2 K_{3}(1+\epsilon) T}+\beta \\
& +r \mathrm{e}^{-r t} T\left\{\beta \mathrm{e}^{2 K_{3}(1+\epsilon) T}+2 F K_{3}(1+\epsilon) \mathrm{e}^{2 K_{3}(1+\epsilon) T}\right\},
\end{aligned}
$$

where the underlined terms are the same as in the nondissipative case (C.2). Summing up $\left\|\nabla_{\mathbf{x}} \widetilde{\zeta}\right\|_{\widehat{\mathcal{C}^{s}}}$ and $\|\widetilde{\zeta}\|_{\widehat{\mathcal{C}^{s}}}$ results in $(6.34 \mathrm{a})$, where the constant $\beta$ in the formula for $A$ is multiplied by 2 for convenience (that is the estimate is more conservative than required).

To derive (6.34b), let $\widetilde{\zeta}=\mathrm{e}^{-r t} \widehat{\zeta}=\mathrm{e}^{-r t}\left\{\widehat{\zeta}_{1}+r \widehat{\zeta}_{2}\right\}$, where

$$
\begin{aligned}
& \widehat{\zeta}_{1}=\widehat{\zeta}^{I}(\xi(0 ; x, y, t), \eta(0 ; x, y, t))+\beta\left[\eta(0 ; x, y, t)-\mathrm{e}^{r t} y\right] \\
& \widehat{\zeta}_{2}=\int_{0}^{t}\left\{\beta \eta(\tau ; x, y, t)+F(-1)^{i}\left[\widetilde{\psi}_{1}-\widetilde{\psi}_{2}\right](\xi(\tau ; x, y, t), \tau)\right\} \mathrm{e}^{r \tau} \mathrm{d} \tau
\end{aligned}
$$

Next, $\partial_{t} \widetilde{\zeta}=-r \mathrm{e}^{-r t} \widehat{\zeta}+\mathrm{e}^{-r t} \partial_{t} \widehat{\zeta}$, where

$$
\begin{aligned}
\partial_{t} \widehat{\zeta}_{1}= & \nabla_{\xi} \widetilde{\zeta}^{I} \cdot \partial_{t} \xi+\beta\left(\partial_{t} \eta-r \mathrm{e}^{r t} y\right) \\
\partial_{t} \widehat{\zeta}_{2}= & \beta \eta \mathrm{e}^{r t}+\beta \int_{0}^{t} \frac{\partial \eta}{\partial t}(\tau ; x, y, t) \mathrm{e}^{r \tau} \mathrm{d} \tau \\
& +F(-1)^{i}\left\{\left(\widetilde{\psi}_{1}-\widetilde{\psi}_{2}\right) \mathrm{e}^{r t}+\int_{0}^{t} \nabla_{\xi}\left(\widetilde{\psi}_{1}-\widetilde{\psi}_{2}\right)\left(\xi_{t}, \eta_{t}\right) \mathrm{e}^{r \tau} \mathrm{d} \tau\right\} .
\end{aligned}
$$

This yields

$$
\begin{aligned}
\left\|\partial_{t} \widetilde{\zeta}\right\|_{\widehat{\mathcal{C}}^{s}} \leqq & \frac{L_{1} K_{3}(1+\epsilon) \mathrm{e}^{2 K_{3}(1+\epsilon) T}+r\|\widehat{\zeta}\|_{\widehat{\mathcal{C}^{s}}}+r \beta \mathrm{e}^{r T}}{}+r\left[\beta \mathrm{e}^{r T}+2 \beta K_{3}(1+\epsilon) \mathrm{e}^{2 K_{3}(1+\epsilon) T} \mathrm{e}^{r T} T+2 F K_{3}(1+\epsilon) \mathrm{e}^{r T}\right. \\
& \left.+2 F K_{3}(1+\epsilon) \cdot K_{3}(1+\epsilon) \mathrm{e}^{2 K_{3}(1+\epsilon) T} \mathrm{e}^{r T} T\right],
\end{aligned}
$$


where the first term comes from $\mathrm{e}^{-r t} \partial_{t} \widehat{\zeta}_{1}$, that is, the nondissipative case, the second from $-r \mathrm{e}^{-r t} \widehat{\zeta}$, the third from $-\beta r \mathrm{e}^{r t} y$, and the terms in square brackets originate from $\partial_{t} \widehat{\zeta}_{2}$. Note, that

$$
\|\widehat{\zeta}\|_{\widehat{\mathcal{C}}^{s}}=\left\|\widehat{\zeta}^{I}\right\|_{\widehat{\mathcal{C}}^{s+1}}+2 \beta+r T \mathrm{e}^{r T}\left\{\beta+2 F K_{3}(1+\epsilon)\right\},
$$

as derived above. This altogether gives (6.34b).

\section{References}

1. Abarbanel, H.D.I., Holm, D.D., Marsden, J.E., Ratiu, T.S.: Nonlinear stability analysis of stratified fluid equilibria. Phil. Trans. R. Soc. Lond. A 318, 349-409 (1986)

2. Adams, R.A.: Sobolev spaces. Academic Press, New York, 1978

3. Adrianova, L.YA.: Introduction to linear systems of differential equations. American Mathematical Society, Providence, RI, 1995

4. Arnold, V.: Sur la géométrie différentielle des groupes de Lie de dimension infinie et ses applications a l'hydrodynamique des fluides parfaits. Ann. Inst. Fourier 16, 319-361 (1966)

5. ARNOLD, V.I.: On conditions for non-linear stability of plane stationary flows of an ideal fluid. Soviet Math. Dokl. 6, 773-777 (1965)

6. ARnOLD, V.I.: On an apriori estimate in the theory of hydrodynamic stability. Am. Math. Soc. Transl. 19, 267-269 (1969)

7. Bennett, A.F., Kloeden, P.E.: The periodic quasigeostrophic equations: existence and uniqueness of strong solutions. Proc. R. Soc. Edinb. A 91, 185-203 (1982)

8. Bernstein, S.N.: Sur la généralisation du probléme de Dirichlet. Math. Ann. 62, 253-272 (1906)

9. Bloch, A., Krishnaprasad, P.S., Marsden, J.E., Ratiu, T.S.: Dissipation induced instabilities. Anal. Nonlineaire Ann. Inst. H. Poincaré 11, 37-90 (1994)

10. Bourgeous, A.J., BeAle, J.T.: Validity of the quasigeostrophic model for large-scale flow in the atmosphere and ocean. SIAM J. Math. Anal. 25, 1023-1068 (1994)

11. Burton, G.R.: Global nonlinear stability for steady ideal fluid flow in bounded planar domains. Arch. Rat. Mech. Anal. 176, 149-163 (2005)

12. CARR, J.: Applications of centre manifold theory. Springer, New York, 1981

13. CARR, J., Muncaster, R.G.: The application of centre manifolds to amplitude expansions. II. Infinite dimensional problems. J. Differ. Equ. 50, 280-288 (1983)

14. Charney, J.G., Eliassen, A.: A numerical method for predicting the perturbations of the middle latitude westerlies. Tellus 1, 38-54 (1949)

15. Cherry, T.M.: Some examples of trajectories defined by differential equations of a generalized dynamical type. Trans. Cambridge Philos. Soc. 23, 165-200 (1925)

16. Chetayev, N.G.: The stability of motion. Pergamon Press, New York, 1961

17. Chorin, A.J., Marsden, J.E.: A mathematical introduction to fluid dynamics. Springer, New York, 1993

18. Clere, M.G., Marsden, J.E.: Dissipation-induced instabilities in an optical cavity laser: A mechanical analog near the 1:1 resonance. Phys. Rev. E 64, 067603 (2001)

19. Colin, T.: The Cauchy problem and the continuous limit for the multilayer model in geophysical fluid dynamics. SIAM J. Math. Anal. 28, 516-529 (1997)

20. Courant, R., Hilbert, D.: Methods of mathematical physics. II. Interscience, New York, 1962

21. Cross, M.C., Hohenberg, P.C.: Pattern formation outside of equilibrium. Rev. Mod. Phys. 65, 851-1112 (1993)

22. Daleckit, Ju.L., Krein, M.G.: Stability of solutions of differential equations in Banach space. American Mathematical Society, Providence, RI, 1974

23. Demidovich, B.P.: Lectures on mathematical theory of stability. Nauka, Moscow, 1967 
24. Dutton, J.A.: The nonlinear quasi-geostrophic equation: existence and uniqueness of solutions on a bounded domain. J. Atmos. Sci. 31, 422-433 (1974)

25. EADY, E.T.: Long waves and cyclone waves. Tellus 1, 38-52 (1949)

26. EbIn, D.G., MARSDEN, J.: Groups of diffeomorphisms and the motion of an incompressible fluid. Ann. Math. 92, 102-163 (1970)

27. Engel, K.-J., NAGEL, R.: One-parameter semigroups for linear evolution equations. Springer, New York, 1999

28. Friedlander, S., Pavlović, N., Shvydkoy, R.: Nonlinear instability for the NavierStokes equations. Comm. Math. Phys. 264, 335-347 (2006)

29. Friedlander, S., Strauss, W., Vishik, M.: Nonlinear instability in an ideal fluid. Ann. Inst. Henri Poincaré 14, 187-209 (1997)

30. Friedlander, S., Yudovich, V.: Instabilities in fluid motion. Notices Am. Math. Soc. 46, 1358-1367 (1999)

31. Friedman, A.: Partial differential equations. Holt, Rinehart, and Winston, New York, 1969

32. Gallay, Th.: A center-stable manifold theorem for differential equations in Banach spaces. Commun. Math. Phys. 152, 249-268 (1993)

33. Gibion, J.D., McGuinness, M.J.: A derivation of the Lorentz equations for some unstable dispersive physical systems. Phys. Lett. A 77, 295-299 (1980)

34. Gilbarg, D., Trudinger, N.S.: Elliptic partial differential equations of second order. Springer, New York, 2001

35. Hadamard, J.: Sur les problèmes aux dérivées partielles et leur signification physique. Princeton University Bulletin, pp. 49-52, 1902

36. Hagerty, P., Bloch, A.M., Weinstein, M.I.: Radiation induced instability in interconnected systems. Proceedings of the 38th CDC, IEEE, pp. 651-656, 1999

37. Hart, J.E.: Finite amplitude baroclinic instability. Ann. Rev. Fluid Mech. 11, 147-172 (1979)

38. Hartman, P.: Ordinary differential equations. SIAM, Philadelphia, 2002

39. Henry, D.: Geometric theory of semilinear parabolic equations. Springer, New York, 1981

40. Holm, D.D.: Hamiltonian formulation of the baroclinic quasigeostrophic fluid equations. Phys. Fluids 29, 7-8 (1986)

41. Holm, D.D., Marsden, J.E., Ratiu, T., Weinstein, A.: Nonlinear stability of fluid and plasma equilibria. Phys. Reports 123, 1-116 (1985)

42. Holopainen, E.O.: On the effect of friction in baroclinic waves. Tellus 13, 363-367 (1961)

43. KaPITSA, P.L.: Stability and transition through the critical speed of fast rotating shafts with friction. Zhur. Tekhn. Fiz. 9, 124-147 (1939)

44. Kato, T.: On classical solutions of the two-dimensional non-stationary Euler equation. Arch. Rat. Mech. Anal. 25, 188-200 (1967)

45. Kato, T., Fujita, H.: On the nonstationary Navier-Stokes system. Rend. Sem. Mat. Univ. Padova 32, 243-260 (1962)

46. Klainerman, S., Majda, A.: Singular limits of quasilinear hyperbolic systems with large parameters and the incompressible limit of compressible fluids. Comm. Pure Appl. Math. 43, 481-524 (1981)

47. Krechetnikov, R., Marsden, J.E.: On destabilizing effects of two fundamental non-conservative forces. Physica D 214, 25-32 (2006)

48. Krechetnikov, R., Marsden, J.E.: Dissipation-induced instabilities in finite dimensions. Rev. Mod. Phys. 79, 519-553 (2007)

49. WalefFe F.: Transitions in shear flows. Nonlinear normality versus non-normal linearity. Phys. Fluids 7, 3060-3066 (1995)

50. KreIn, S.G.: Linear differential equations in Banach space. American Mathematical Society, Providence, RI, 1972. (first published in Russian in 1963)

51. Krylov, N.V.: Lectures on elliptic and parabolic equations in Hölder spaces. American Mathematical Society, Providence, RI, 1996 
52. Ladyzhenskaya, O.A., URAL'TSEVA, N.N.: Linear and quasilinear elliptic equations. Academic Press, New York, 1968

53. LewIS, D.: Bifurcation of liquid drops. Nonlinearity 6, 491-522 (1993)

54. Lewis, D., Marsden, J.E., RatiU, T.: Stability and bifurcation of a rotating planar liquid drop. J. Math. Phys. 28, 2508-2515 (1987)

55. Lin, C.C.: The theory of hydrodynamic stability. Cambridge University Press, New York, 1955

56. Luo, Z.-H., Guo, B.-Z., Morgul, O.: Stability and stabilization of infinite dimensional systems with applications. Springer, London, 1999

57. Lyapunov, A.: The general problem of the stability of motion. Taylor and Francis, London, 1992

58. Majda, A.J., Bertozzi, A.L.: Vorticity and incompressible flow. Cambridge University Press, New York, 2002

59. McIntyre, M.E., Shepherd, T.G.: An exact local conservation theorem for finiteamplitude disturbances to non-parallel shear flows, with remarks on Hamiltonian structure ans on Arnol'd's stability theorems. J. Fluid Mech. 181, 527-565 (1987)

60. Merkin, D.R.: Gyroscopic systems. Gostekhizdat, Moscow, 1956 (in Russian)

61. Merkin, D.R.: Introduction to the theory of stability. Springer, New York, 1997

62. Mielke, A.: On the energetic stability of solitary water waves. Phil. Trans. R. Soc. Lond. A 360, 2337-2358 (2002)

63. Miranda, C.: Partial differential equations of elliptic type. Springer, Berlin, 1970

64. Morrison, P.J.: Hamiltonian description of the ideal fluid. Rev. Mod. Phys. 70 467-521 (1998)

65. Nikolai, E.L.: Theoretical Mechanics. GONTI, Moscow, 1939 (in Russian)

66. Nirenberg, L.: On elliptic partial differential equations. Ann. Scuola Norm. Sup. Pisa 13, 115-162 (1959)

67. PAZY, A.: Semigroups of linear operators and applications to partial differential equations. Springer, New York, 1983

68. Pedlosky, J.: Finite-amplitude baroclinic waves. J. Atmos. Sci. 27, 15-30 (1970)

69. Pedlosky, J.: Finite-amplitude baroclinic waves with small dissipation. J. Atmos. Sci. 28, 587-597 (1971)

70. Pedlosky, J.: Geophysical fluid dynamics. Springer, New York, 1987

71. Phillips, N.A.: A simple three-dimensional model for the study of large scale extratropical flow patterns. J. Meteor. 8, 381-394 (1951)

72. Phillips, N.A.: Energy transformations and meridional circulations associated with simple baroclinic waves in a two-level, quasi-geostrophic model. Tellus 6, 273-286 (1954)

73. Pierini, S., VulPiani, A.: Nonlinear stability analysis in multilayer quasigeostrophic systems. J. Phys. A 14, L203-L207 (1981)

74. Pierrehumbert, R.T., Swanson, K.L.: Baroclinic instability. Annu. Rev. Fluid Mech. 27, 419-467 (1995)

75. Romea, R.A.: The effects of friction and $\beta$ on finite-amplitude baroclinic waves. J. Atmos. Sci. 34, 1689-1695 (1977)

76. Rossby, C.G., Collaborators.: Relations between variations in the intensity of the zonal circulation of the atmosphere and the displacements of the semipermanent centers of action. J. Marine Res. 2, 38-55 (1939)

77. Saijo, M., Gourgoulhon, E.: Viscosity driven instability in rotating relativistic stars. Phys. Rev. D 74, 084006 (2006)

78. Salmon, R.: Lectures on geophysical fluid dynamics. Oxford University Press, New York, 1998

79. SchaudER, J.P.: Über lineare elliptische Differentialgleichungen sweiter Ordnung. Math. Z. 38, 257-282 (1934)

80. ShePherd, T.G.: Symmetries, conservation laws, and Hamiltonian structure in geophysical fluid dynamics. Adv. Geophys. 32, 287-338 (1990)

81. Smart, D.R.: Fixed point theorems. Cambridge University Press, London, 1974 
82. Swaters, G.E.: A nonlinear stability theorem for baroclinic quasigeostrophic flow. Phys. Fluids 29, 5-6 (1986)

83. Thomson, W., Tait, P.G.: Treatise on Natural Philosophy: Part 1. Cambridge University Press, Cambridge, 1879

84. Thorne, K., Prince, R., MacDonald, D.: Black Holes: the membrane paradigm. Yale University Press, New Haven, 1986

85. Wan, Y.H., Pulvirenti, M.: Nonlinear stability of circular vortex patches. Comm. Math. Phys. 99, 435-450 (1985)

86. Weiland, J., Wilhelmsson, H.: Coherent nonlinear interaction of waves in plasmas. Pergamon Press, Oxford, 1977

87. Wirn-Nielsen, A.: Nonlinear studies of quasi-geostrophic systems. Physica D 77, 33-59 (1994)

88. Yudovich, V.I.: The linearization method in hydrodynamical stability theory. American Mathematical Society, Providence, RI, 1989

Control and Dynamical Systems,

California Institute of Technology,

Pasadena, CA 91125, USA.

e-mail: marsden@cds.caltech.edu

and

Present address:

Department of Mechanical Engineering, University of California at Santa Barbara,

Santa Barbara, CA 93106, USA.

e-mail: rkrechet@engineering.ucsb.edu

(Received 7 July 2005 / Accepted 30 June 2008)

Published online January 13, 2009 - (C) Springer-Verlag (2009) 